\title{
CALIBRAÇÃO CONTROLADA \\ APLICADA NA QUÍMICA ANALÍTICA
}

\author{
Betsabé Grimalda Blas Achic
}

DISSERTAÇÃO APRESENTADA AO INSTITUTO DE MATEMÁTICA E ESTATÍSTICA DA UNIVERSIDADE DE SÃO PAULO PARA OBTENÇÃO DO TÍTULO DE MESTRE EM ESTATÍSTICA

Área de Concentração: Estatística

Orientadora: Profa. Dra. Mônica Carneiro Sandoval

Durante a elaboração deste trabalho a autora

recebeu apoio financeiro do Instituto de Pesquisas Tecnológicas(IPT)

São Paulo, dezembro de 2005 


\section{Calibração controlada aplicada na química analítica}

Este exemplar corresponde à redação final da dissertação devidamente corrigi da e defendida por Betsabé Grimalda Blas Achic e aprovada pela comissão julgadora.

São Paulo, dezembro de 2005.

Banca examinadora:

. Profa. Dra. Mônica Carneiro Sandoval (orientadora) (IME-USP)

. Prof. Dr. Heleno Bolfarine (IME-USP)

. Profa. Dra. Olga Satomi Yoshida (IPT) 


\section{Dedicatória}

A meus pais e meus irmãos. 


\section{Agradecimentos}

Gostaria de expressar minha gratidão a todos que tornaram possível o desenvolvimento desse trabalho.

Em primeiro lugar, gostaria de agradecer à minha orientadora Profa. Dra. Mônica Carneiro Sandoval pela confiança e especialmente pelo constante apoio durante o desenvolvimento dessa dissertação. Pela motivação que tem me ajudado ter confiança durante este trabalho e pelas esclarecedoras sugestões que ajudaram muito a resolver problemas. Também, pela paciência na cuidadosa revisão dessa dissertação.

A Dra. Olga Satomi Yoshida quem propôs o interessante tema de tópicos avançados de cálculo de incertezas e modelo de calibração estatística, para dados reais na química analítica, o qual foi o tópico principal na minha dissertação. Também, pela orientação no Instituto de Pesquisas Tecnológicas (IPT).

A Sra. Elaine de Oliveira de santos pela explicação do processo de preparação das soluções-padrão, método de espectrometria de plasma e absorção atômica no laboratório de química inorgánica do IPT. E pelos dados fornecidos para o Capítulo 5.

A Dra. Alice Sakuma, Karina Pretto e Roberta Okura, pelas discussões no tema de preparação das soluções-padrão. A Dra. Alice Sakuma pelos dados fornecidos para o Capítulo 5. 
Ao Prof. Dr. Carlos Pereira por ter motivado o inicio de leitura de artigos científicos relacionados à minha dissertação na disciplina de Probabilidade e Inferência II. Ao Prof. Dr. Luís Gustavo pela discussão para o entendimento do artigo de Welch (1950), artigo importante para o Capítulo 4. Ao Dr. Manoel Galea Rojas pela discussão no tema do modelo de calibração controlada.

Agradeço também, a todos os professores e ao Departamento de Estatística do IMEUSP. Gostaria agradecer, também, ao IPT pelo apoio financiero durante os meus estudos do mestrado.

Meus agradecimentos seriam incompletos se eu deixar de mencionar nomes de parentes e meus amigos.

A meus pais, Sr. Eusebio Blas Rubina e Sra. Grimalda Achic Alarcón, quem foram compreensivos e a apesar da distância me deram motivação e coragem para alcançar meus objetivos. E a meus irmãos Harold, Yudy, Wilhem, Evert e Hegel.

Ao Cristian Bayes, Iván, Joan, Ester, Susana, Núbia, Lourdes, Cecilia, Pedro, Caio, Augusto, Marcos, Luis Fernando, Bruno, Marina, Karina Valdívia, Christian Paz, Jesús, Patricia e Alexandre, pela amizade, companheirismo e boa disposição que sempre tiveram conmigo. 


\section{Resumo}

O modelo de calibração usual, utilizado para estimar a concentração de um elemento químico presente em uma amostra, supõe que a variável independente é fixada e isenta de erro. No entanto, em aplicações na química analítica, essa variável está sujeita a erros provenientes do processo de preparação das soluções-padrão. Neste trabalho, propomos modelos de calibração homoscedástico e heteroscedástico que além de incorporar o erro na variável consideram observações controladas. Apresentamos métodos para obtenção de incertezas expandidas associadas à concentração das soluções-padrão. Ilustramos e comparamos os modelos propostos com o modelo usual por meio de estudos de simulação e aplicações utilizando conjunto de dados reais. 


\section{Abstract}

The calibration usual model is used to estimate the concentration of a chemical especie in a given test sample, it assumes that the independent variable is fixed and is not subject to error. However, in applications in analytical chemistry this variable is subject to error arised from the preparation process of standard solution. In this work, we propose the homoscedastic and heteroscedastic calibration models that incorporate errors of the independent variables associated to controlled observations. We present methods to compute expanded uncertainty associated to the concentration of standard solution. We illustrate and compare the proposed models with the usual model through simulation studies and by applications using real data set. 


\section{Índice}

1 Introdução

2 Modelo de calibração

2.1 Calibração em química analítica . . . . . . . . . . . . 5

2.2 Modelo de calibração usual $\ldots \ldots \ldots \ldots \ldots \ldots \ldots \ldots$

2.2 .1 Estimador clássico . . . . . . . . . . . . . 8

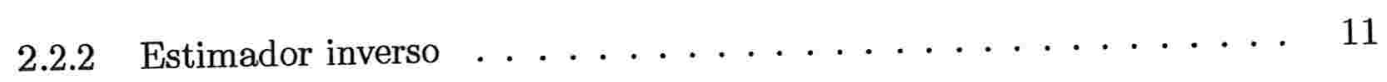

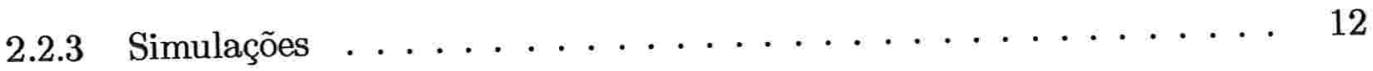

3 Modelo de calibração para observações controladas $\quad 19$

3.1 Processo de preparação da amostra . . . . . . . . . . . . . 19

3.2 Processo de preparação das soluções-padrão $\ldots \ldots \ldots \ldots \ldots \ldots \ldots$

3.3 Modelo de calibração controlada . . . . . . . . . . . . . . 23

3.3.1 Modelo homoscedástico . . . . . . . . . . . . . . . . 24

3.3 .2 Modelo heteroscedástico . . . . . . . . . . . . 28 
Índice

3.4 Simulações . . . . . . . . . . . . . . . . . 31

4 Comparação dos métodos de cálculo de incerteza expandida

4.1 Lei de propagação de incertezas . . . . . . . . . . . . . . 55

4.1 .1 Exemplo.......................... 57

4.2 Métodos para o cálculo de incertezas expandidas . . . . . . . . 58

4.2.1 Incerteza expandida obtida por meio da solução em séries . . . . . 6 60

4.2.2 Incerteza expandida recomendada pelo guia ISOGUM . . . . . . 62

4.3 Comparação dos métodos para obter as incertezas expandidas . . . . . . . 63

5 Aplicações

5.1 Aplicação do cálculo de incerteza . . . . . . . . . . . . . . . 67

5.2 Aplicação do modelo heteroscedástico $\ldots \ldots \ldots \ldots \ldots \ldots$. . . . . . 70

6 Comentários finais 
CAPÍtUlo 1

\section{Introdução}

A Química Analítica é uma área científica que desenvolve e aplica métodos, instrumentos e estratégias para obter informações sobre a composição da matéria no espaço e no tempo. Para isso, dois aspectos estão envolvidos: a identificação das espécies presentes e a determinação das quantidades relativas de cada uma dessas espécies (Danzer e Kurrie, 1998). A análise qualitativa compreende os ensaios que permitem ao químico identificar elementos presentes em uma amostra. Já, a análise quantitativa compreende as técnicas e métodos para determinação das quantidades dos componentes na amostra.

A metrologia, ciência de medições, foi muito desenvolvida pelos físicos. Segundo King (1997) particularmente, o cálculo de incertezas é novo para a maioria dos químicos analíticos. As dificuldades existentes entre metrologistas e químicos analíticos em parte são devidas ao uso de diferentes conceitos e terminologias. No ano de 1993 a Organização Internacional para Padronizações (ISO) juntamente com outras organizações internacionais elaboraram o "Guide to the Expression of Uncertainty in Measurement"(conhecido como ISOGUM ou GUM ) com o objetivo de estabelecer um conjunto de recomendações e regras gerais para expressar incertezas das medições para uso internacional na área da metrologia. Uma versão do ISOGUM em português foi publicada pelo INMETRO (ISOGUM, 1998). Um guia muito utilizado na área química é o chamado guia EURACHEM (EURACHEM/CITAC, 2002), que mostra como os conceitos do guia ISOGUM podem ser aplicados à medição química. 
A motivação para o desenvolvimento deste trabalho foi o estudo dos processos rotineiros do laboratório de química inorgânica do Instituto de Pesquisas Tecnológicas (IPT), com o objetivo de contribuir na melhora do desempenho da análise quantitativa. O interesse nessa análise é estimar a concentração de uma determinada espécie química em uma amostra e a sua incerteza.

Uma das preocupações das medições químicas é o cálculo da incerteza estatística associada aos resultados obtidos. Os laboratórios analíticos prestadores de serviço necessitam ter resultados cada vez mais exatos e precisos, e para esse propósito deve-se representar o processo analítico mediante um modelo que contenha maior informação possível.

Para estimar a concentração, métodos analíticos como espectrometria de absorção atômica ou espectrometria de plasma são utilizados. Inicialmente, são avaliadas as respostas (absorbância ou intensidade, respectivamente) fornecidas por esses métodos para quantidades pré-fixadas das soluções-padrão (solução com concentração pré-fixada). Posteriormente, essa resposta é, também, avaliada para uma quantidade desconhecida de uma solução-amostra e, finalmente, mediante um modelo de calibração estima-se essa quantidade desconhecida e sua incerteza.

Os modelos de calibração consistem em dois estágios. No primeiro estágio, chamado de experimento de calibração, os valores de uma variável aleatória dependente correspondentes a $n$ valores pré-fixados de uma variável aleatória independente são observados de modo a se estimar a função que relaciona as duas variáveis. No segundo estágio, a calibração propriamente dita, são observados $k$ valores da variável aleatória dependente correspondentes a um valor desconhecido da variável independente. O interesse é estimar esse valor desconhecido levando-se em conta as informações dos dois estágios.

Na química analítica, para estimar a concentração de um elemento químico presente em uma amostra, o guia EURACHEM recomenda o uso do modelo de calibração usual supondo que a variável independente é fixada e isenta de erro. Mas, sabe-se que o processo de preparação das soluções-padrão acarreta uma incerteza na obtenção da concentração 
requerida para essas soluções.

O objetivo de nosso trabalho é:

- Estudar os métodos para estimar a incerteza da concentração das soluções-padrão.

- Propor um modelo de calibração que considere os erros das soluções-padrão.

Este trabalho é composto por mais cinco capítulos. O conteúdo desses capítulos são resumidos a seguir.

No Capítulo 2, falamos brevemente sobre calibração em química analítica e apresentamos o modelo de calibração usual (recomendado pelo guia EURACHEM) e os estimadores clássico e inverso obtidos a partir desse modelo. Realizamos, também, alguns estudos de simulação.

No Capítulo 3, apresentamos uma breve descrição da preparação da amostra e da solução-padrão. Como no processo de preparação das soluções-padrão não se consegue atingir a quantidade desejada (pré-fixada) pelo químico devido aos erros de medida, nesse capítulo propomos dois modelos de calibração, denominados de modelos homoscedástico e heteroscedástico, que incorporam o erro cometido na preparação das soluções-padrão. O modelo homoscedástico, supõe que as variâncias dos erros das soluções-padrão são iguais. Tratamos os casos em que essa variância é desconhecida e conhecida. Já, o modelo de calibração heteroscedástico considera que as variâncias desses erros dependem da concentração, e estudamos apenas o caso em que essas variâncias são conhecidas. Apresentamos, também, os resultados das simulações considerando o modelo usual e os modelos homoscedásticos propostos.

No Capítulo 4, apresentamos as definições e terminologias utilizadas na química analítica, sugeridas pelo ISOGUM e o guia EURACHEM. Além disso, apresentamos a lei de propagação de incertezas e uma comparação dos métodos de cálculo de incerteza. 
No Capítulo 5, apresentamos uma aplicação dos métodos de cálculo de incerteza discutido no Capítulo 4, utilizando dados fornecidos pelo Instituto Adolfo Lutz. Aplicamos o modelo heteroscedástico apresentado no Capítulo 3 e comparamos os resultados obtidos com os do modelo usual, a partir de dados fornecidos pelo laboratório de química do IPT.

Para finalizar, apresentamos, no Capítulo 6, alguns comentários finais incluindo sugestões para trabalhos futuros. 


\section{Modelo de calibração}

Nesse capítulo, apresentamos o modelo de calibração linear simples atualmente utilizado na química analítica e realizamos alguns estudos de simulação.

\subsection{Calibração em química analítica}

Segundo Danzer e Currie (1998), na química analítica, a calibração, em geral, é uma operação que relaciona uma grandeza de saída com uma grandeza de entrada, para um sistema de medida sobre determinadas condições. Para uma amostra composta de $m$ espécies químicas, temos que as grandezas de entrada caracterizam alguma grandeza específica da espécie química, como número atômico, número de massa ou um valor de energia típico, denominada de $Q_{j}$ e sua concentração $x_{j}, j=1, \cdots, m$. As grandezas de saída são valores medidos, isto é, sinal observado $Y_{j}$ na posição $R_{j}$, por exemplo, para o método de espectrometria de plasma o sinal é a intensidade e para o método de absorção atômica é a absorbância. Existem, portanto, quatro grandezas $Q=\left(Q_{1}, \cdots, Q_{m}\right), x=\left(x_{1}, \cdots, x_{m}\right), R=$ $\left(R_{1}, \cdots, R_{m}\right)$ e $Y=\left(Y_{1}, \cdots, Y_{m}\right)$ que estão relacionadas entre si. A relação entre as grandezas de entrada é chamada de função analítica e a relação entre as grandezas de saída é chamada de função de medida. Na Figura 2.1, o lado esquerdo descreve a função analítica, 


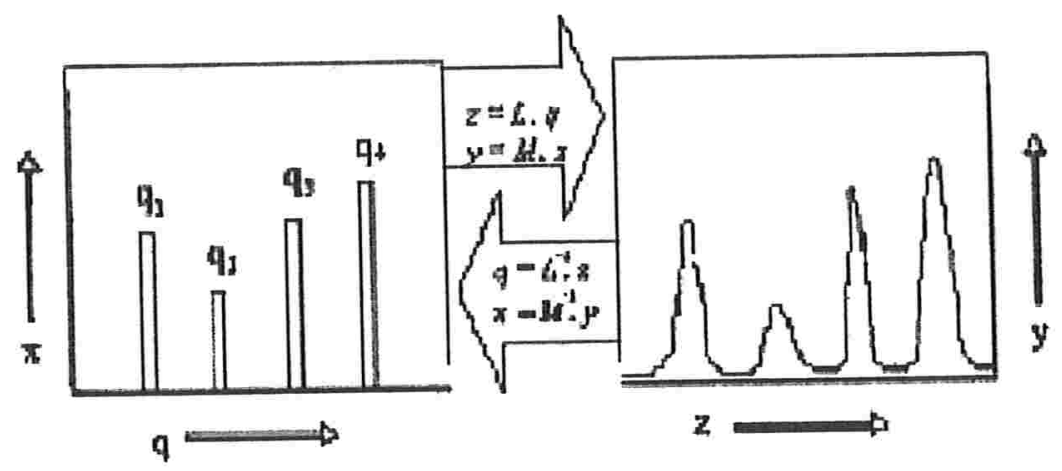

Figura 2.1: Relação entre as grandezas $Q, x, R$ e $Y$.

já o lado direito descreve a função de medida. Podemos estabelecer outras relações, por exemplo, a relação entre a espécie $Q$ e a posição $R$, dada na Figura 2.1 pela equação $R=g_{1}(Q)$. A partir dessa, a relação entre a posição $R$ e a concentração $x$ é estabelecida, e o caso mais comum é a relação entre a concentração $x$ de um analito e o sinal $Y$, dada na Figura 2.1 pela equação $Y=g_{2}(x)$. Devido a erros de medição

$$
Y=g_{2}(x)+\epsilon,
$$

em que $g_{2}(x)$ é conhecida como a função de calibração ou curva de calibração. Muitas vezes, o interesse é conhecer apenas a concentração da espécie química $j$, sendo as outras componentes desprezadas. Nesse caso, temos que $x=x_{j}$ sendo assim, uma variável unidimensional. Nesse trabalho só estamos interessados em estudar o caso unidimensional.

Com o objetivo de estimar a concentração do elemento químico presente na amostra, modelos de calibração devem ser utilizados. Como vimos na Introdução, os modelos de calibração consideram dois estágios, que na química analítica são:

Primeiro estágio: são fixadas $n$ quantidades do analito $x$, chamadas de soluções-padrão e para cada uma o equipamento de espectrometria de plasma (ou absorção atômica) fornece as respostas $Y_{i}, i=1, \cdots, n$ em termos de intensidade (ou absorbância).

Esses dados constituem o experimento de calibração e são usados para estimar a função $g_{2}$ definida em (2.1).

Segundo estágio: são preparadas $k$ soluções-amostra com a mesma concentração desco- 
nhecida $X_{0}$ e obtém-se a intensidade (ou absorbância) $Y_{0 i}, i=n+1, \cdots, n+k$. A partir da curva estimada no primeiro estágio, a concentração $X_{0}$ é estimada .

\subsection{Modelo de calibração usual}

O modelo de calibração usual considera a curva de calibração $g_{2}(X)$ da equação $(2.1)$ como sendo uma função linear e é definido por

$$
\begin{aligned}
Y_{i} & =\alpha+\beta x_{i}+\epsilon_{i}, \quad i=1, \cdots, n, \\
Y_{0 i} & =\alpha+\beta X_{0}+\epsilon_{i}, \quad i=n+1, \cdots, n+k,
\end{aligned}
$$

com as seguintes suposições:

- $x_{1}, \cdots, x_{n}$ são valores fixados;

- os erros $\epsilon_{1}, \cdots, \epsilon_{n+k}$ têm distribuição normal com média zero e variância constante $\sigma_{\epsilon}^{2}$

- os erros são não correlacionados.

Note que os pontos $\left(x_{i}, Y_{i}\right)$, para $i=1, \cdots, n$ correspondem ao primeiro estágio e $\left(X_{0}, Y_{0 i}\right)$, para $i=n+1, \cdots, n+k$, ao segundo estágio. Os parâmetros do modelo são $\alpha, \beta, X_{0}$ e $\sigma_{\epsilon}^{2}$, sendo que o interesse principal é estimar a quantidade $X_{0}$.

Dois estimadores da quantidade $X_{0}$ são discutidos na literatura, o estimador de máxima verossimilhança ou de mínimos quadrados, denominado estimador clássico, e por outro lado, baseado na regressão de $x$ em $Y$, o estimador obtido pelo método de mínimos quadrados, denominado de estimador inverso.

No caso da análise química, $X_{0}$ representa a concentração desconhecida. Pela primeira suposição estamos considerando que a preparação das soluções-padrão é isenta de erro, ou seja, a concentração da i-ésima solução-padrão é exatamente $x_{i}$. 


\subsubsection{Estimador clássico}

Utilizando o método de máxima verossimilhança obtemos os estimadores de $\alpha, \beta, X_{0}$ e $\sigma_{\epsilon}^{2}$ dados respectivamente por

$$
\begin{aligned}
\hat{\alpha} & =\bar{Y}-\hat{\beta} \bar{x} \\
\hat{\beta} & =\frac{\sum_{i=1}^{n}\left(x_{i}-\bar{x}\right)\left(Y_{i}-\bar{Y}\right)}{\sum_{i=1}^{n}\left(x_{i}-\bar{x}\right)^{2}}=\frac{S_{x Y}}{S_{x x}}, \hat{\beta} \neq 0, \\
\hat{X}_{0 C} & =\frac{\bar{Y}_{0}-\hat{\alpha}}{\hat{\beta}} \\
\hat{\sigma}_{\epsilon}{ }^{2} & =\frac{1}{n+k}\left[\sum_{i=1}^{n}\left(Y_{i}-\hat{\alpha}-\hat{\beta} x_{i}\right)^{2}+\sum_{i=n+1}^{n+k}\left(Y_{0 i}-\bar{Y}_{0}\right)^{2}\right],
\end{aligned}
$$

sendo,

$$
\begin{aligned}
\bar{x} & =\frac{1}{n} \sum_{i=1}^{n} x_{i} \\
\bar{Y} & =\frac{1}{n} \sum_{i=1}^{n} Y_{i} \\
S_{x Y} & =\frac{1}{n} \sum_{i=1}^{n}\left(x_{i}-\bar{x}\right)\left(Y_{i}-\bar{Y}\right), \\
S_{x x} & =\frac{1}{n} \sum_{i=1}^{n}\left(x_{i}-\bar{x}\right)^{2} \\
\bar{Y}_{0} & =\frac{1}{k} \sum_{i=n+1}^{n+k} Y_{0 i} .
\end{aligned}
$$

Os estimadores $\hat{\alpha}, \hat{\beta}$ e $\hat{X}_{0}$ também podem ser obtidos utilizando-se o método de mínimos quadrados. Notemos que $\hat{\alpha}$ e $\hat{\beta}$ dependem apenas do primeiro estágio do modelo de calibração usual, então a teoria de regressão linear simples continua válida. Portanto, além de estimadores de máxima verossimilhança (EMV), $\hat{\alpha}$ e $\hat{\beta}$ são estimadores lineares não viciados de variância mínima.

O estimador $\sigma_{\epsilon}^{2}$ é viciado, pois

$$
E\left({\hat{\sigma_{\epsilon}}}^{2}\right)=\frac{n+k-3}{n+k} \sigma_{\epsilon}^{2},
$$


logo, podemos definir um estimador não viciado para $\sigma_{\epsilon}^{2}$, denominado $\hat{\sigma}_{\epsilon_{c}}^{2}$, como

$$
\hat{\sigma}_{\epsilon_{c}}^{2}=\frac{n+k}{n+k-3} \hat{\sigma}_{\epsilon}^{2}=\frac{1}{n+k-3}\left[\sum_{i=1}^{n}\left(Y_{i}-\hat{\alpha}-\hat{\beta} x_{i}\right)^{2}+\sum_{i=n+1}^{n+k}\left(Y_{0 i}-\bar{Y}_{0}\right)^{2}\right] \text {. }
$$

Os estimadores $\hat{\sigma}_{\epsilon}^{2}$ e $\hat{\sigma}_{\epsilon c}^{2}$ dependem dos dois estágios, isso só não ocorre quando $k=1$, ou seja, quando temos uma única observação no segundo estágio. Nas expressões (2.7) e (2.9) temos duas parcelas, isto é,

- $\sum_{i=1}^{n}\left(Y_{i}-\hat{\alpha}-\hat{\beta} x_{i}\right)^{2}$, parcela devida aos erros de ajuste da curva de calibração (comumente conhecida como a soma de quadrados de resíduos) e

- $\sum_{i=n+1}^{n+k}\left(Y_{0 i}-\bar{Y}_{0}\right)^{2}$, parcela devida aos erros de medida da variável $Y_{0}$.

A matriz de informação de Fisher de $\theta=\left(\alpha, \beta, X_{0}, \sigma_{\epsilon}^{2}\right)$ é dada por:

$$
I(\theta)=\frac{1}{\sigma_{\epsilon}^{2}}\left(\begin{array}{cccc}
n+k & k X_{0}+\sum_{i=1}^{n} x_{i} & k \beta & 0 \\
k X_{0}+\sum_{i=1}^{n} x_{i} & k X_{0}^{2}+\sum_{i=1}^{n} x_{i}^{2} & k X_{0} \beta & 0 \\
k \beta & k X_{0} \beta & k \beta^{2} & 0 \\
0 & 0 & 0 & \frac{n+k}{2 \sigma_{\epsilon}^{2}}
\end{array}\right) .
$$

O estimador de máxima verossimilhança $\hat{\theta}=\left(\hat{\alpha}, \hat{\beta}, \hat{X}_{0}, \hat{\sigma}_{\epsilon}^{2}\right)$ tem distribuição aproximadamente normal com média $\theta$ e matriz de covariâncias $I(\theta)^{-1}$ quando $k=q n, q \in Q^{+} \mathrm{e}$ $n \longrightarrow \infty$. Assim, temos que a variância aproximada de ordem $n^{-1}$ de $\hat{X}_{0}$ é dada por:

$$
\begin{aligned}
V_{1}\left(\hat{X}_{0 C}\right) & =\frac{\sigma_{\epsilon}^{2}}{k \beta^{2}}\left[\frac{(n+k)\left(k X_{0}^{2}+\sum_{i=1}^{n} x_{i}^{2}\right)-\left(k X_{0}+\sum_{i=1}^{n} x_{i}\right)^{2}}{n S_{x x}}\right] \\
& =\frac{\sigma_{\epsilon}^{2}}{\beta^{2}}\left[\frac{1}{k}+\frac{1}{n}+\frac{\left(\bar{x}-X_{0}\right)^{2}}{n S_{x x}}\right] .
\end{aligned}
$$

Shukla (1972), considerando $k$ fixo e expandindo $\hat{X}_{0 C}$, expressão (2.6), em série de Taylor no ponto $(\alpha, \beta)$ obteve as seguintes aproximações, ignorando os termos de ordem menor que $n^{-2}$, para o valor esperado, a variância, o vício e o erro quadrático médio (EQM) 
$\operatorname{de} \hat{X}_{0 C}$ :

$$
\begin{aligned}
E\left(\hat{X}_{0 C}\right) & =X_{0}+\frac{\sigma_{\epsilon}^{2}\left(X_{0}-\bar{x}\right)}{n \beta^{2} S_{x x}} \\
V_{2}\left(\hat{X}_{0 C}\right) & =\frac{\sigma_{\epsilon}^{2}}{\beta^{2}}\left[\frac{1}{n}+\frac{1}{k}+\frac{\left(\bar{x}-\hat{X}_{0 C}\right)^{2}}{n S_{x x}}+\frac{3 \sigma_{\epsilon}^{2}}{k n \beta^{2} S_{x x}}\right], \\
\operatorname{Ví} \operatorname{cio}\left(\hat{X}_{0 C}\right) & =\frac{\sigma_{\epsilon}^{2}\left(X_{0}-\bar{x}\right)}{n \beta^{2} S_{x x}} \\
\operatorname{EQM}\left(\hat{X}_{0 C}\right) & =\frac{\sigma_{\epsilon}^{2}}{\beta^{2}}\left[\frac{1}{n}+\frac{1}{k}+\frac{\left(\bar{x}-\hat{X}_{0 C}\right)^{2}}{n S_{x x}}+\frac{3 \sigma_{\epsilon}^{2}}{k n \beta^{2} S_{x x}}\right] .
\end{aligned}
$$

Podemos observar que o estimador $\hat{X}_{0 C}$ de $X_{0}$ é viciado, mas é assintoticamente não viciado, pois $E\left(\hat{X}_{0 C}\right)=X_{0 C}$ quando $n \longrightarrow \infty$.

Com relação à variância do estimador $\hat{X}_{0 C}$, notemos que, quando $k=q n, q \in Q^{+} \mathrm{e}$ ignorando os termos de ordem menor que $n^{-1}$, a variância obtida por Shukla em (2.12) coincide com a variância dada em (2.10), encontrada através da informação de Fisher. A equação (2.10) considera tamanhos de amostras grandes na primeira e segunda etapa ( $n$ e $k$ ), já a equação (2.12) considera amostra grande apenas na primeira etapa e uma amostra de tamanho fixo na segunda etapa.

Lwin (1981) estendeu esses resultados para o modelo sem a suposição de normalidade dos erros, somente considerando que a distribuição de $\epsilon$ pertence a uma familia de distribuições com $E(\epsilon)=0, E\left(\epsilon^{2}\right)=\sigma_{\epsilon}^{2}, E\left(\epsilon^{3}\right)$ e $E\left(\epsilon^{4}\right)$ finitas.

Para construir um intervalo de confiança para $X_{0}$ consideramos que

$$
\frac{\hat{X}_{0 C}-X_{0}}{\hat{V}\left(\hat{X}_{0 C}\right)} \stackrel{D}{\longrightarrow} N(0,1)
$$

em que $\hat{V}\left(\hat{X}_{0 C}\right)$ é a variância dada em (2.10) ou (2.12) estimada. Logo, o intervalo de confiança aproximado para $X_{0}$ com um nível de confiança igual a $(1-\alpha)$ é dado por

$$
\left(\hat{X}_{0 C}-z_{\frac{\alpha}{2}} \sqrt{\hat{V}\left(\hat{X}_{0 C}\right)}, \hat{X}_{0 C}+z_{\frac{\alpha}{2}} \sqrt{\hat{V}\left(\hat{X}_{0 C}\right)}\right),
$$

em que $z_{\frac{\alpha}{2}}$ é o quantil de ordem $\left(1-\frac{\alpha}{2}\right)$ da distribuição normal padrão. 


\subsubsection{Estimador inverso}

O estimador inverso é obtido da regressão de $x$ em $Y$, apesar de $x$ não ser variável aleatória. Podemos reescrever o modelo (2.2) como:

$$
x_{i}=\gamma+\phi Y_{i}+\epsilon_{i}^{\prime}, \quad 1=1, \cdots, n,
$$

sendo $\epsilon_{i}^{\prime}=-\epsilon_{i} \phi, \gamma=-\frac{\alpha}{\beta}$, e $\phi=\frac{1}{\beta}$.

Os estimadores de $\gamma$ e $\phi$ obtidos pelo método de mínimos quadrados são dados, respectivamente, por

$$
\begin{aligned}
& \hat{\gamma}=\bar{x}+\hat{\phi} \bar{Y} \\
& \hat{\phi}=\frac{\frac{1}{n} \sum_{i=1}^{n}\left(x_{i}-\bar{x}\right)\left(Y_{i}-\bar{Y}\right)}{\sum_{i=1}^{n}\left(Y_{i}-\bar{Y}\right)^{2}}=\frac{S_{x Y}}{S_{Y Y}} .
\end{aligned}
$$

Notemos que $\hat{\gamma}=-\frac{\hat{\alpha}}{\hat{\hat{\beta}}}$ e $\hat{\phi}=\frac{1}{\hat{\boldsymbol{\beta}}}$.

Assim, o estimador inverso, $\hat{X}_{0 I}$, é dado por

$$
\hat{X}_{0 I}=\hat{\gamma}+\hat{\phi} \bar{Y}_{0}
$$

Shukla (1972), considerando $k$ fixo, obteve também, aproximações de ordem $n^{-1}$ para o valor esperado, a variância, o vício e o erro quadrático médio (EQM) de $\hat{X}_{0 I}$ que são dadas por

$$
\begin{aligned}
E\left(\hat{X}_{0 I}\right)= & X_{0}+\frac{(n-1) \sigma_{\epsilon}^{2}\left(\bar{x}-X_{0}\right)}{n \beta^{2} S_{x x} \rho}-\frac{2(n-1) \sigma_{\epsilon}^{2}\left(\bar{x}-X_{0}\right)}{n^{2} \beta^{2} S_{x x} \rho^{3}}, \\
V\left(\hat{X}_{0 I}\right)= & \frac{\sigma_{\epsilon}^{2}}{\beta^{2} \rho^{2}}\left[\frac{1}{n}+\frac{1}{k}+\frac{\left(\bar{x}-\hat{X}_{0 I}\right)^{2}}{n S_{x x}}+\frac{\sigma_{\epsilon}^{2}\left(\rho^{2}-2 \rho+6\right)}{k n S_{x x} \beta^{2} \rho^{2}}\right]-\frac{2 \sigma_{\epsilon}^{4}\left(\bar{x}-\hat{X}_{0 I}\right)^{2}}{\left(n^{3} /(n-1)^{2}\right) \rho^{4} \beta^{4} S_{x x}^{2}}, \\
\operatorname{Ví} \operatorname{cio}\left(\hat{X}_{0 I}\right)= & \frac{(n-1) \sigma_{\epsilon}^{2}\left(\bar{x}-X_{0}\right)}{n \beta^{2} S_{x x} \rho}-\frac{2(n-1) \sigma_{\epsilon}^{2}\left(\bar{x}-X_{0}\right)}{n^{2} \beta^{2} S_{x x} \rho^{3}}, \\
\operatorname{EQM}\left(\hat{X}_{0 I}\right)= & \frac{\sigma^{2}}{\beta^{2} \rho^{2}}\left[\frac{1}{n}+\frac{1}{k}+\frac{\left(\bar{x}-\hat{X}_{0 I}\right)^{2}}{n S_{x x}}+\frac{\sigma^{2}\left(\rho^{2}-2 \rho+6\right)}{k n S_{x x} \beta^{2} \rho^{2}}\right] \\
& -\frac{\sigma^{4}\left(\bar{x}-\hat{X}_{0 I}\right)^{2}}{\left(n^{2} /(n-1)^{2}\right) \rho^{2} \beta^{4} S_{x x}^{2}}\left(1-\frac{6}{n \rho^{2}}\right)
\end{aligned}
$$

sendo $\rho=1+\frac{\sigma^{2}}{\beta^{2}(n /(n-1)) S_{x x}}$. 
Notemos que o estimador inverso $\hat{X}_{0 I}$ é viciado mesmo assintoticamente e só terá vicio nulo quando $X_{0}=\bar{x}$.

Muitos pesquisadores tentaram estabelecer, adotando diversos critérios de comparação, propriedades estatísticas dos estimadores clássico e inverso, sendo bastante discutidas as limitações de cada estimador em função dos tamanhos da amostra no primeiro e segundo estágio. Williams (1969) provou que o erro quadrático médio do estimador clássico é infinito para $n \geq 4$, em que $n$ é o tamanho da amostra no primeiro estágio. Berkson (1969) e Shukla (1972) mostraram que o estimador clássico é consistente e que o erro quadrático médio assintótico do estimador clássico é menor que o do inverso, exceto quando o parâmetro de interesse está próximo à média dos valores iniciais de $x$. Outros pesquisadores com o seu próprio critério de comparação chegaram às mesmas conclusões de Berkson e Shukla. Shukla (1972) concluiu também que, quando n é pequeno, o estimador inverso produz um erro quadrático médio menor que o estimador clássico, particularmente para interpolação. Já, quando o número $k$, tamanho da amostra no segundo estágio, é grande, é improvável que o método inverso seja mais vantajoso que o método clássico. Os principais resultados podem ser encontrados em Brown (1993).

\subsubsection{Simulações}

Como vimos na Seção 2.2.1, temos duas aproximações para a variância de $\hat{X}_{0 C}$. O guia EURACHEM recomenda o uso da expressão (2.10), no entanto, a expressão (2.12) obtida por Shukla (1972) parece ser mais adequada pois considera $k$ fixo.

Nesta seção, nosso objetivo é verificar o comportamento dessas duas aproximações e estudar as propriedades dos estimadores de máxima verossimilhança em amostras finitas. Avaliamos, também, o desempenho dessas variâncias na porcentagem de cobertura e comprimento dos intervalos de confiança.

Para a obtenção dos resultados utilizamos o pacote "MATHCAD 2001 Professional". 
Foram geradas 5000 amostras do modelo de calibração usual dado em (2.2) e (2.3). Em todas as amostras os valores utilizados para os parâmetros $\alpha$ e $\beta$ foram 0,1 e 2, respectivamente. Consideramos a curva de calibração na faixa de concentração $[0,2]$. Os valores das concentrações padrões foram $x_{1}=0, x_{i}=x_{i-1}+\frac{2}{n-1}, i=2, \cdots, n$, e os valores considerados para o parâmetro $X_{0}$ foram 0,01 (valor no extremo inferior), 0,8 (valor próximo à média) e 1,9 (valor no extremo superior). Para $\sigma_{\epsilon}^{2}$ utilizamos dois valores, 0,04 considerada como uma variância "pequena", e 0,4 como uma variância "grande". Os tamanhos das amostras nos dois estágios do modelo de calibração foram $n=5,20,100$ e $k=2,20,100$.

Os vícios médios empíricos são dados por $\sum_{j=1}^{5000}(\hat{\psi}-\psi) / 5000$ e os erros quadráticos médios (EQMs) empíricos são dados por $\sum_{j=1}^{5000}(\hat{\psi}-\psi)^{2} / 5000$, em que $\hat{\psi}$ é um estimador de $\psi=\alpha$ ou $\beta$ ou $X_{0}$. A média das variâncias estimadas de $\hat{X}_{0}$ são dadas por $\sum_{j=1}^{5000} \hat{V}\left(\hat{X}_{0 C}\right) / 5000$, em que $\hat{V}\left(\hat{X}_{0 C}\right)=\hat{V}_{1}\left(\hat{X}_{0 C}\right)$ ou $\hat{V}_{2}\left(\hat{X}_{0 C}\right)$, sendo $\hat{V}_{1}\left(\hat{X}_{0 C}\right)$ e $\hat{V}_{2}\left(\hat{X}_{0 C}\right)$ as estimativas das variáveis obtidas a partir das expressões (2.10) e (2.12), respectivamente. As variâncias teóricas de $\hat{X}_{0 C}$ denotadas por $V_{1}\left(\hat{X}_{0 C}\right)$ e $V_{2}\left(\hat{X}_{0 C}\right)$, referem-se, respectivamente, às expressões (2.10) e (2.12) avaliadas nos parâmetros.

A Tabela 2.1 mostra o vício médio e o EQM empírico dos estimadores de $\alpha$ e $\beta$. Observando essa tabela, verificamos que os valores dos vícios e EQMs empíricos para $\sigma_{\epsilon}^{2}=0,4$ são maiores quando comparados com os resultados para $\sigma_{\epsilon}^{2}=0,04$. Como $\hat{\alpha}$ e $\hat{\beta}$ não dependem do segundo estágio, os resultados para $X_{0}=0,01 ; 0,8 ; 1,9$ são próximos e não variam com $k$. Observamos, também, que os EQMs diminuem com o aumento do tamanho da amostra do primeiro estágio.

Nas Tabelas 2.2 e 2.3 apresentamos os valores do vício e EQM empírico, a média das variâncias estimadas e as variâncias teóricas do estimador clássico, $\hat{X}_{0 C}$. Analisando a Tabela 2.2 observamos que os valores teóricos das variâncias do Shukla $\left(V_{2}\left(\hat{X}_{0}\right)\right)$ e do guia $\left(V_{1}\left(\hat{X}_{0}\right)\right)$ coincidem. Na Tabela 2.3 verificamos que esses valores são próximos, sendo que a variância do Shukla é um pouco maior do que a do guia quando a amostra do segundo estágio é pequena. Esse mesmo comportamento acontece com a variância estimada. As 
tabelas mostram, também, que o vício médio e o EQM diminuem quando aumentamos o tamanho das amostras dos dois estágios. Além disso, o EQM e as variâncias teóricas estão próximos, principalmente quando a amostra do primeiro estágio é grande. Observa-se ainda que os vícios médios e EQM da Tabela 2.3 são maiores quando comparados aos da Tabela 2.2 .

A Tabela 2.4 apresenta as porcentagens de cobertura e amplitude dos intervalos de confiança para o parâmetro $X_{0}$. Os intervalos foram construídos com nível de confiança de $95 \%$ segundo (3.21), em que $\hat{V}\left(\hat{X}_{0 C}\right)=\hat{V}_{1}\left(\hat{X}_{0 C}\right)$ ou $\hat{V}_{2}\left(\hat{X}_{0 C}\right)$ e foram denominados como "Guia"e "Shukla", respectivamente. Analisando essa tabela observamos que as porcentagens de cobertura e as amplitudes dos intervalos de confiança "Guia"e "Shukla" são sempre semelhantes. Verificamos que para todos os valores considerados de $X_{0}, n, \sigma_{\epsilon}^{2}$ e $k$, com exceção de $n=5$ e $k=2$, a porcentagem de cobertura está próxima de $95 \%$. No entanto, notamos que as amplitudes aumentam à medida que aumentamos a variância $\sigma_{\epsilon}^{2}$ e diminuem à medida que aumentamos os tamanhos das amostras do primeiro e segundo estágio. 
Tabela 2.1: Vício e erro quadrático médio empírico de $\hat{\alpha}$ e $\hat{\beta}$.

\begin{tabular}{|c|c|c|c|c|c|c|c|c|c|c|}
\hline \multirow{3}{*}{$X_{0}$} & \multirow{3}{*}{$n$} & \multirow{3}{*}{$k$} & \multicolumn{4}{|c|}{$\sigma_{\epsilon}^{2}=0,04$} & \multicolumn{4}{|c|}{$\sigma_{\epsilon}^{2}=0,4$} \\
\hline & & & \multicolumn{2}{|c|}{$\hat{\alpha}$} & \multicolumn{2}{|c|}{$\hat{\boldsymbol{\beta}}$} & \multicolumn{2}{|c|}{$\hat{\alpha}$} & \multicolumn{2}{|c|}{$\hat{\boldsymbol{\beta}}$} \\
\hline & & & Vício & EQM & Vício & EQM & Vício & EQM & Vício & EQM \\
\hline \multirow[t]{9}{*}{0,01} & \multirow[t]{3}{*}{5} & 2 & 0,0007 & 0,0245 & $-0,0001$ & 0,0163 & $-0,0120$ & 0,2313 & 0,0029 & 0,1535 \\
\hline & & 20 & $-0,0022$ & 0,0237 & 0,0017 & 0,0163 & $-0,0016$ & 0,2445 & $-0,0019$ & 0,1580 \\
\hline & & 100 & $-0,0004$ & 0,0237 & 0,0002 & 0,0158 & $-0,0025$ & 0,2387 & 0,0045 & 0,1583 \\
\hline & \multirow[t]{3}{*}{20} & 2 & $-0,0002$ & 0,0075 & $-0,0004$ & 0,0054 & $-0,0032$ & 0,0752 & 0,0045 & 0,0544 \\
\hline & & 20 & 0,0000 & 0,0075 & $-0,0002$ & 0,0054 & 0,0036 & 0,0737 & $-0,0014$ & 0,0540 \\
\hline & & 100 & 0,0007 & 0,0075 & $-0,0003$ & 0,0054 & 0,0027 & 0,0753 & $-0,0039$ & 0,0550 \\
\hline & \multirow[t]{3}{*}{100} & 2 & 0,0005 & 0,0015 & $-0,0004$ & 0,0012 & $-0,0012$ & 0,0159 & 0,0029 & 0,0119 \\
\hline & & 20 & 0,0008 & 0,0016 & $-0,0005$ & 0,0012 & $-0,0045$ & 0,0158 & 0,0045 & 0,0120 \\
\hline & & 100 & 0,0009 & 0,0016 & $-0,0005$ & 0,0012 & 0,0017 & 0,0163 & $-0,0012$ & 0,0122 \\
\hline \multirow[t]{9}{*}{0,8} & \multirow[t]{3}{*}{5} & 2 & $-0,0022$ & 0,0243 & 0,0032 & 0,0158 & 0,0028 & 0,2428 & $-0,0005$ & 0,1586 \\
\hline & & 20 & $-0,0018$ & 0,0241 & 0,0031 & 0,0156 & 0,0018 & 0,2415 & 0,0015 & 0,1609 \\
\hline & & 100 & 0,0017 & 0,0245 & $-0,0014$ & 0,0164 & $-0,0002$ & 0,2418 & 0,0027 & 0,1616 \\
\hline & \multirow[t]{3}{*}{20} & 2 & 0,0010 & 0,0076 & $-0,0004$ & 0,0054 & 0,0014 & 0,0755 & 0,0008 & 0,0550 \\
\hline & & 20 & $-0,0003$ & 0,0077 & 0,0003 & 0,0056 & $-0,0043$ & 0,0742 & 0,0031 & 0,0542 \\
\hline & & 100 & $-0,0013$ & 0,0073 & 0,0013 & 0,0053 & 0,0064 & 0,0743 & $-0,0014$ & 0,0531 \\
\hline & \multirow[t]{3}{*}{100} & 2 & 0,0004 & 0,0015 & $-0,0006$ & 0,0011 & 0,0022 & 0,0159 & $-0,0011$ & 0,0121 \\
\hline & & 20 & 0,0013 & 0,0016 & $-0,0010$ & 0,0012 & 0,0019 & 0,0159 & $-0,0015$ & 0,0121 \\
\hline & & 100 & 0,0006 & 0,0016 & $-0,0007$ & 0,0012 & $-0,0022$ & 0,0156 & 0,0023 & 0,0119 \\
\hline \multirow[t]{9}{*}{1,9} & \multirow[t]{3}{*}{5} & 2 & 0,0029 & 0,0235 & $-0,0004$ & 0,0159 & 0,0100 & 0,2438 & $-0,0071$ & 0,1621 \\
\hline & & 20 & 0,0000 & 0,0240 & $-0,0004$ & 0,0160 & 0,0072 & 0,2479 & $-0,0030$ & 0,1646 \\
\hline & & 100 & 0,0017 & 0,0236 & 0,0005 & 0,0156 & $-0,0145$ & 0,2438 & 0,0101 & 0,1644 \\
\hline & \multirow[t]{3}{*}{20} & 2 & 0,0030 & 0,0073 & $-0,0021$ & 0,0054 & $-0,0058$ & 0,0730 & 0,0021 & 0,0526 \\
\hline & & 20 & 0,0011 & 0,0074 & $-0,0011$ & 0,0054 & 0,0037 & 0,0739 & $-0,0020$ & 0,0526 \\
\hline & & 100 & 0,0010 & 0,0074 & $-0,0010$ & 0,0054 & $-0,0025$ & 0,0733 & 0,0013 & 0,0537 \\
\hline & \multirow[t]{3}{*}{100} & 2 & $-0,0006$ & 0,0016 & 0,0006 & 0,0012 & $-0,0026$ & 0,0161 & 0,0035 & 0,0121 \\
\hline & & 20 & 0,0000 & 0,0016 & $-0,0001$ & 0,0012 & $-0,0013$ & 0,0158 & 0,0017 & 0,0116 \\
\hline & & 100 & 0,0000 & 0,0016 & 0,0002 & 0,0012 & $-0,0014$ & 0,0159 & 0,0018 & 0,0119 \\
\hline
\end{tabular}


Tabela 2.2: Vício e erro quadrático médio empírico, média das variâncias estimadas e variância teórica de $\hat{X}_{0}$, para $\sigma_{\epsilon}^{2}=0,04$.

\begin{tabular}{|c|c|c|c|c|c|c|c|c|}
\hline \multirow[t]{2}{*}{$X_{0}$} & \multirow[t]{2}{*}{$n$} & \multirow[t]{2}{*}{$k$} & \multicolumn{2}{|c|}{ Empírico } & \multicolumn{2}{|c|}{ Variância estimada } & \multicolumn{2}{|c|}{ Variância teórica } \\
\hline & & & Vício & EQM & $\hat{V}_{1}\left(\hat{X}_{0}\right)$ & $\hat{V}_{2}\left(\hat{X}_{0}\right)$ & $V_{1}\left(\hat{X}_{0}\right)$ & $V_{2}\left(\hat{X}_{0}\right)$ \\
\hline \multirow[t]{9}{*}{0,01} & 5 & 2 & $-0,0032$ & 0,0109 & 0,0063 & 0,0064 & 0,0109 & 0,0110 \\
\hline & & 20 & $-0,0034$ & 0,0064 & 0,0059 & 0,0059 & 0,0064 & 0,0064 \\
\hline & & 100 & $-0,0036$ & 0,0061 & 0,0060 & 0,0060 & 0,0060 & 0,0060 \\
\hline & 20 & 2 & $-0,0022$ & 0,0067 & 0,0059 & 0,0059 & 0,0068 & 0,0069 \\
\hline & & 20 & $-0,0017$ & 0,0024 & 0,0022 & 0,0022 & 0,0023 & 0,0023 \\
\hline & & 100 & $-0,0017$ & 0,0020 & 0,0019 & 0,0019 & 0,0019 & 0,0019 \\
\hline & 100 & 2 & $-0,0001$ & 0,0056 & 0,0053 & 0,0053 & 0,0054 & 0,0054 \\
\hline & & 20 & $-0,0010$ & 0,0009 & 0,0009 & 0,0009 & 0,0009 & 0,0009 \\
\hline & & 100 & $-0,0007$ & 0,0005 & 0,0005 & 0,0005 & 0,0005 & 0,0005 \\
\hline \multirow[t]{9}{*}{0,80} & 5 & 2 & $-0,0016$ & 0,0072 & 0,0041 & 0,0041 & 0,0072 & 0,0072 \\
\hline & & 20 & $-0,0016$ & 0,0027 & 0,0024 & 0,0024 & 0,0027 & 0,0027 \\
\hline & & 100 & $-0,0012$ & 0,0023 & 0,0022 & 0,0022 & 0,0023 & 0,0023 \\
\hline & 20 & 2 & $-0,0003$ & 0,0055 & 0,0048 & 0,0048 & 0,0056 & 0,0056 \\
\hline & & 20 & $-0,0003$ & 0,0011 & 0,0010 & 0,0010 & 0,0011 & 0,0011 \\
\hline & & 100 & $-0,0002$ & 0,0006 & 0,0006 & 0,0006 & 0,0007 & 0,0007 \\
\hline & 100 & 2 & $-0,0003$ & 0,0053 & 0,0050 & 0,0050 & 0,0051 & 0,0051 \\
\hline & & 20 & $-0,0004$ & 0,0006 & 0,0006 & 0,0006 & 0,0006 & 0,0006 \\
\hline & & 100 & $-0,0002$ & 0,0002 & 0,0002 & 0,0002 & 0,0002 & 0,0002 \\
\hline \multirow[t]{9}{*}{1,90} & 5 & 2 & 0,0030 & 0,0099 & 0,0061 & 0,0061 & 0,0102 & 0,0103 \\
\hline & & 20 & 0,0031 & 0,0059 & 0,0052 & 0,0052 & 0,0057 & 0,0057 \\
\hline & & 100 & 0,0022 & 0,0053 & 0,0053 & 0,0053 & 0,0053 & 0,0053 \\
\hline & 20 & 2 & 0,0022 & 0,0065 & 0,0057 & 0,0058 & 0,0066 & 0,0066 \\
\hline & & 20 & 0,0021 & 0,0021 & 0,0020 & 0,0020 & 0,0021 & 0,0021 \\
\hline & & 100 & 0,0015 & 0,0017 & 0,0017 & 0,0017 & 0,0017 & 0,0017 \\
\hline & 100 & 2 & $-0,0013$ & 0,0053 & 0,0052 & 0,0052 & 0,0053 & 0,0053 \\
\hline & & 20 & 0,0009 & 0,0008 & 0,0008 & 0,0008 & 0,0008 & 0,0008 \\
\hline & & 100 & 0,0002 & 0,0005 & 0,0004 & 0,0004 & 0,0004 & 0,0004 \\
\hline
\end{tabular}


Tabela 2.3: Vício e erro quadrático médio empírico, média das variâncias estimadas e variância teórica de $\hat{X}_{0}$, para $\sigma_{\epsilon}^{2}=0,4$.

\begin{tabular}{|c|c|c|c|c|c|c|c|c|}
\hline \multirow[t]{2}{*}{$X_{0}$} & \multirow[t]{2}{*}{$n$} & \multirow[t]{2}{*}{$k$} & \multicolumn{2}{|c|}{ Empírico } & \multicolumn{2}{|c|}{ Variância estimada } & \multicolumn{2}{|c|}{ Variância teórica } \\
\hline & & & Vício & EQM & $\hat{V}_{1}\left(\hat{X}_{0}\right)$ & $\hat{V}_{2}\left(\hat{X}_{0}\right)$ & $V_{1}\left(\hat{X}_{0}\right)$ & $V_{2}\left(\hat{X}_{0}\right)$ \\
\hline \multirow[t]{9}{*}{0,01} & \multirow[t]{3}{*}{5} & 2 & $-0,0366$ & 0,1351 & 0,0845 & 0,0892 & 0,1092 & 0,1152 \\
\hline & & 20 & $-0,0434$ & 0,0920 & 0,0859 & 0,0868 & 0,0642 & 0,0648 \\
\hline & & 100 & $-0,0440$ & 0,0847 & 0,0908 & 0,0910 & 0,0602 & 0,0603 \\
\hline & \multirow[t]{3}{*}{20} & 2 & $-0,0077$ & 0,0716 & 0,0633 & 0,0653 & 0,0683 & 0,0703 \\
\hline & & 20 & $-0,0173$ & 0,0254 & 0,0243 & 0,0245 & 0,0233 & 0,0235 \\
\hline & & 100 & $-0,0161$ & 0,0218 & 0,0215 & 0,0216 & 0,0193 & 0,0193 \\
\hline & \multirow[t]{3}{*}{100} & 2 & $-0,0033$ & 0,0542 & 0,0526 & 0,0531 & 0,0539 & 0,0543 \\
\hline & & 20 & 0,0000 & 0,0091 & 0,0088 & 0,0088 & 0,0089 & 0,0089 \\
\hline & & 100 & $-0,0037$ & 0,0052 & 0,0049 & 0,0049 & 0,0049 & 0,0049 \\
\hline \multirow[t]{9}{*}{0,80} & \multirow[t]{3}{*}{5} & 2 & $-0,0138$ & 0,0846 & 0,0510 & 0,0562 & 0,0716 & 0,0776 \\
\hline & & 20 & $-0,0085$ & 0,0312 & 0,0292 & 0,0300 & 0,0266 & 0,0272 \\
\hline & & 100 & $-0,0114$ & 0,0263 & 0,0276 & 0,0278 & 0,0226 & 0,0227 \\
\hline & \multirow[t]{3}{*}{20} & 2 & $-0,0056$ & 0,0575 & 0,0509 & 0,0529 & 0,0555 & 0,0576 \\
\hline & & 20 & $-0,0020$ & 0,0108 & 0,0103 & 0,0105 & 0,0105 & 0,0107 \\
\hline & & 100 & $-0,0062$ & 0,0071 & 0,0068 & 0,0069 & 0,0065 & 0,0066 \\
\hline & \multirow[t]{3}{*}{100} & 2 & $-0,0040$ & 0,0509 & 0,0502 & 0,0507 & 0,0511 & 0,0516 \\
\hline & & 20 & 0,0009 & 0,0062 & 0,0061 & 0,0061 & 0,0061 & 0,0062 \\
\hline & & 100 & 0,0001 & 0,0021 & 0,0021 & 0,0021 & 0,0021 & 0,0021 \\
\hline \multirow[t]{9}{*}{1,90} & \multirow[t]{3}{*}{5} & 2 & 0,0464 & 0,1333 & 0,0836 & 0,0891 & 0,1024 & 0,1084 \\
\hline & & 20 & 0,0426 & 0,0805 & 0,0791 & 0,0800 & 0,0574 & 0,0580 \\
\hline & & 100 & 0,0393 & 0,0744 & 0,0777 & 0,0779 & 0,0534 & 0,0535 \\
\hline & \multirow[t]{3}{*}{20} & 2 & 0,0092 & 0,0703 & 0,0610 & 0,0629 & 0,0660 & 0,0680 \\
\hline & & 20 & 0,0099 & 0,0222 & 0,0215 & 0,0218 & 0,0210 & 0,0212 \\
\hline & & 100 & 0,0125 & 0,0182 & 0,0185 & 0,0186 & 0,0170 & 0,0170 \\
\hline & \multirow[t]{3}{*}{100} & 2 & 0,0014 & 0,0543 & 0,0524 & 0,0528 & 0,0534 & 0,0538 \\
\hline & & 20 & 0,0012 & 0,0082 & 0,0083 & 0,0083 & 0,0084 & 0,0084 \\
\hline & & 100 & 0,0015 & 0,0044 & 0,0044 & 0,0044 & 0,0044 & 0,0044 \\
\hline
\end{tabular}


Tabela 2.4: Porcentagem de cobertura (\%) e amplitude (A) dos intervalos de $95 \%$ de confiança para o parâmetro $X_{0}$.

\begin{tabular}{|c|c|c|c|c|c|c|c|c|c|c|}
\hline \multirow{3}{*}{$X_{0}$} & \multirow{3}{*}{$n$} & \multirow{3}{*}{$k$} & \multicolumn{4}{|c|}{$\sigma_{\epsilon}^{2}=0,04$} & \multicolumn{4}{|c|}{$\sigma_{\epsilon}^{2}=0,4$} \\
\hline & & & \multicolumn{2}{|c|}{ Guia } & \multicolumn{2}{|c|}{ Shukla } & \multicolumn{2}{|c|}{ Guia } & \multicolumn{2}{|c|}{ Shukla } \\
\hline & & & $\%$ & $\mathrm{~A}$ & $\%$ & A & $\%$ & $\mathrm{~A}$ & $\%$ & A \\
\hline \multirow[t]{9}{*}{0,01} & \multirow[t]{3}{*}{5} & 2 & 79,76 & 0,29 & 79,76 & 0,29 & 80,92 & 1,02 & 81,40 & 1,05 \\
\hline & & 20 & 92,12 & 0,29 & 92,12 & 0,29 & 92,32 & 1,05 & 92,38 & 1,05 \\
\hline & & 100 & 94,40 & 0,30 & 94,40 & 0,30 & 94,22 & 1,06 & 94,22 & 1,06 \\
\hline & \multirow[t]{3}{*}{20} & 2 & 91,56 & 0,30 & 91,58 & 0,30 & 92,04 & 0,96 & 92,28 & 0,98 \\
\hline & & 20 & 92,76 & 0,18 & 92,76 & 0,18 & 93,52 & 0,59 & 93,58 & 0,59 \\
\hline & & 100 & 94,54 & 0,17 & 94,54 & 0,17 & 94,38 & 0,56 & 94,40 & 0,56 \\
\hline & \multirow[t]{3}{*}{100} & 2 & 93,84 & 0,28 & 93,90 & 0,28 & 94,30 & 0,90 & 94,44 & 0,90 \\
\hline & & 20 & 93,64 & 0,12 & 93,64 & 0,12 & 94,24 & 0,37 & 94,26 & 0,37 \\
\hline & & 100 & 94,50 & 0,09 & 94,50 & 0,09 & 94,08 & 0,27 & 94,12 & 0,27 \\
\hline \multirow[t]{9}{*}{0,8} & \multirow[t]{3}{*}{5} & 2 & 78,98 & 0,24 & 79,02 & 0,24 & 79,52 & 0,80 & 80,04 & 0,83 \\
\hline & & 20 & 92,48 & 0,19 & 92,54 & 0,19 & 94,12 & 0,64 & 94,22 & 0,65 \\
\hline & & 100 & 94,76 & 0,18 & 94,76 & 0,18 & 96,18 & 0,62 & 96,18 & 0,63 \\
\hline & \multirow[t]{3}{*}{20} & 2 & 91,80 & 0,27 & 91,80 & 0,27 & 91,78 & 0,86 & 92,00 & 0,88 \\
\hline & & 20 & 93,40 & 0,12 & 93,44 & 0,12 & 94,16 & 0,39 & 94,30 & 0,40 \\
\hline & & 100 & 94,20 & 0,10 & 94,22 & 0,10 & 95,04 & 0,32 & 95,08 & 0,32 \\
\hline & \multirow[t]{3}{*}{100} & 2 & 94,52 & 0,28 & 94,52 & 0,28 & 95,10 & 0,87 & 95,20 & 0,88 \\
\hline & & 20 & 94,98 & 0,10 & 94,98 & 0,10 & 93,98 & 0,30 & 94,06 & 0,30 \\
\hline & & 100 & 94,66 & 0,06 & 94,66 & 0,06 & 94,38 & 0,18 & 94,38 & 0,18 \\
\hline \multirow[t]{9}{*}{1,9} & \multirow[t]{3}{*}{5} & 2 & 79,82 & 0,29 & 79,88 & 0,29 & 81,02 & 0,98 & 81,44 & 1,01 \\
\hline & & 20 & 92,32 & 0,28 & 92,34 & 0,28 & 92,68 & 0,99 & 92,78 & 0,99 \\
\hline & & 100 & 94,22 & 0,28 & 94,22 & 0,28 & 94,44 & 1,01 & 94,44 & 1,01 \\
\hline & \multirow[t]{3}{*}{20} & 2 & 91,56 & 0,29 & 91,56 & 0,29 & 92,02 & 0,94 & 92,40 & 0,96 \\
\hline & & 20 & 93,24 & 0,17 & 93,26 & 0,17 & 93,84 & 0,56 & 93,94 & 0,56 \\
\hline & & 100 & 94,68 & 0,16 & 94,68 & 0,16 & 94,70 & 0,52 & 94,70 & 0,52 \\
\hline & \multirow[t]{3}{*}{100} & 2 & 95,14 & 0,28 & 95,14 & 0,28 & 94,18 & 0,90 & 94,22 & 0,90 \\
\hline & & 20 & 94,90 & 0,11 & 94,96 & 0,11 & 94,62 & 0,36 & 94,64 & 0,36 \\
\hline & & 100 & 94,44 & 0,08 & 94,44 & 0,08 & 94,74 & 0,26 & 94,76 & 0,26 \\
\hline
\end{tabular}


CAPÍTUlO 3

\section{Modelo de calibração para}

\section{observações controladas}

Nesse capítulo, apresentamos uma breve descrição da preparação da amostra e da soluçãopadrão. O processo de preparação das soluções-padrão acarreta uma incerteza na obtenção da concentração requerida para essas soluções. O modelo de calibração usual apresentado no Capítulo 2 não contempla a existência dessa incerteza, e na literatura não se tem um modelo de calibração que descreva o processo de medições utilizado na análise química. Alguns modelos de calibração (homoscedástico e heteroscedástico) que consideram o erro da solução-padrão são propostos. Para finalizar o capítulo desenvolvemos estudos de simulação.

\subsection{Processo de preparação da amostra}

Uma amostra, chamada de amostra bruta ou amostra geral, é derivada de um lote e é fornecida pelo cliente do laboratório.

No laboratório a amostra bruta é transformada em amostra laboratorial seguindo o processo estabelecido pelas normas. Por exemplo, uma amostra bruta sólida pode passar por um procedimento que a transforma em partículas pequenas ou em pó fino. 
Da amostra laboratorial pequenas porções são pesadas numa balança analítica ${ }^{1}$ tentandose obter aproximadamente a mesma massa (geralmente, estão em um intervalo de 0,2 a $1 \mathrm{~g}$ ). As amostras pesadas são transformadas em estado líquido, chamada solução-amostra, para então serem analisadas. Por exemplo: deseja-se analisar o elemento sódio (Na) de um determinado material, então três amostras são obtidas da amostra laboratorial tentando-se obter $0,25 \mathrm{~g}$. As três amostras fornecem os pesos $0,2528 \mathrm{~g} ; 0,2523 \mathrm{~g}$ e $0,2522 \mathrm{~g}$ respectivamente. Depois, cada amostra é transformada na forma de solução.

O processo de transformação das massas pesadas na forma de solução é feito de acordo com o tipo de amostra. Por exemplo, em um determinado processo, num primeiro passo são adicionados ácidos tais como o ácido sulfúrico, fluorídrico, nítrico ou clorídrico, nas correspondentes amostras pesadas. Esta amostra acidificada (num béquer) é levada na chapa quente por um determinado tempo e a uma temperatura adequada. Após esfriado o béquer, o conteúdo é transferido para um balão volumétrico de $250-\mathrm{mL}$ e para obter-se a solução-amostra acrescenta-se água. Se for necessário são feitas diluições apropriadas para, finalmente, essas alíquotas, de concentração $X_{0}$, serem lidas pelo equipamento.

\subsection{Processo de preparação das soluções-padrão}

Suponha que o químico quer obter $n$ soluções-padrão com concentração fixada, $c_{2 i}, i=$ $1, \cdots, n$ (essas concentrações serão utilizadas no primeiro estágio do modelo de calibração, com $X_{i}=c_{2 i}$ ). Vejamos como esse processo é realizado: o fabricante fornece ao laboratório o material de referência tendo uma concentração estimada $c_{0}(\mathrm{mg} / \mathrm{L})$ e uma incerteza $u\left(c_{0}\right)$. Pipeta-se uma quantidade $p_{0}$ cuja incerteza é $u\left(p_{0}\right)$, transfere-se a um balão e adicionamse os reagentes, resultando um volume $b_{0}$ (sendo a sua incerteza $u\left(b_{0}\right)$ ). Deste último balão são pipetadas pequenas quantidades $p_{i}, i=1, \ldots, n$, (cuja incerteza é $u\left(p_{i}\right), i=$ $1, \ldots, n$, respectivamente) que são transferidas a $n$ balões. Os balões são completados

\footnotetext{
${ }^{1}$ Balança que possui em uma sensibilidade pelo menos igual a $0,1 \mathrm{mg}$.
} 
com reagentes convenientemente a fim de obter os volumes $b_{i}, i=1, \ldots, n$, (sendo a sua incerteza $u\left(b_{i}\right), i=1, \ldots, n$, respectivamente), para se conseguir as soluções-padrão com as concentrações desejadas.

Em cada fase do processo o que se quer medir é a densidade (massa/volume). Na fase 1 , tem-se que a concentração no balão $b_{0}$ é

$$
c_{1}=\frac{c_{0} p_{0}}{b_{0}}
$$

já na fase 2, temos que a concentração é

$$
c_{2 i}=\frac{c_{1} p_{i}}{b_{i}}
$$

ou

$$
c_{2 i}=\frac{c_{0} p_{0} p_{i}}{b_{0} b_{i}}
$$

Note que tem-se um erro proveniente do material de referência $u\left(c_{0}\right)$ e outros erros, $u\left(p_{0}\right), u\left(p_{i}\right), u\left(b_{0}\right), u\left(b_{i}\right)$, gerados dentro do processo, portanto a concentração $c_{2 i}$ não está isenta de erro.

Vejamos, graficamente, a explicação do processo de preparação do padrão.

O material de referência pode ser líquido ou sólido. Quando é sólido tem que ser transformado em solução de acordo com as indicações do fabricante.

O seguinte gráfico mostra o processo realizado na fase 1 para o caso em que o material de referência é sólido. 


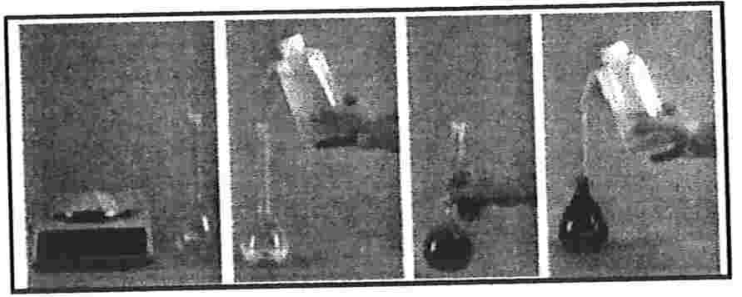

Figura 3.1: Transformação do material de referência (Fase 1)

Os passos seguidos no gráfico acima são: primeiramente o soluto é pesado, a seguir é transferido ao balão, onde adiciona-se o solvente e agita-se suavemente para obter-se uma solução homogênea. São extraídas pequenas quantidades para a preparação das soluçõespadrão.

As fases 1 e 2 são resumidas no seguinte gráfico:

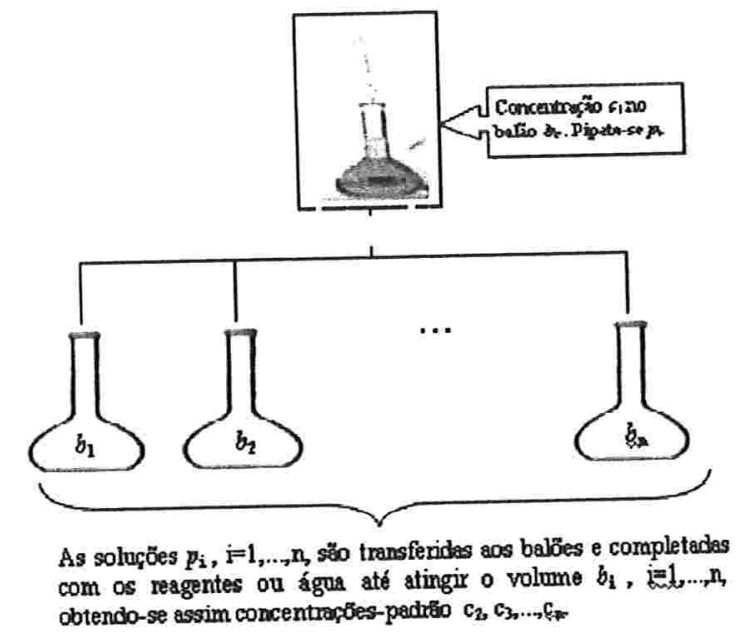

Figura 3.2: Preparação das soluções-padrão 


\subsection{Modelo de calibração controlada}

A variável independente $x$ da equação 2.2 representa, na análise química, as verdadeiras concentrações das soluções-padrão, mas como apresentamos na Seção 3.2, o processo de preparação das soluções-padrão envolve erros de medida, o que leva a não obtenção da verdadeira concentração.

Existem várias situações práticas em que a variável independente $x$ é medida com erro. Nesse caso, segundo Berkson (1950) torna-se necessário definir o tipo de observação: controlada e não controlada.

As observações são ditas não controladas quando o procedimento utilizado para se obter o verdadeiro valor da variável independente $x_{i}$ produz um erro e o valor observado passa a ser

$$
X_{i}=x_{i}+\delta_{i}
$$

Temos que $x_{i}$ é uma quantidade fixada, porém desconhecida, enquanto que o erro $\delta_{i}$ e, conseqüentemente, $X_{i}$ são variáveis aleatórias. O modelo definido por (2.2) e (3.4) é chamado de modelo com erro nas variáveis (Fuller, 1987) e nesse caso, existe correlação entre os erros do modelo e a variável $X_{i}$. Já, o modelo definido por (2.2), (2.3) e (3.4) é chamado de modelo de calibração com erro nas variáveis (Lima, 1996).

As observações são ditas controladas quando um valor $X_{i}$ é pré-definido de acordo com a conveniência do experimento e um procedimento é estabelecido tentando-se atingir esse valor. Esse experimento fornece $x_{i}$, mas que não é observado e é tal que

$$
x_{i}=X_{i}-\delta_{i}
$$

Nesse caso, a quantidade fixada é $X_{i}$ e o erro $\delta_{i}$ e $x_{i}$ são variáveis aleatórias. O modelo definido por (2.2) e (3.5) é conhecido como modelo de Berkson e nesse caso, os erros do modelo e $X_{i}$ são independentes.

Estamos interessados em estudar o caso onde as concentrações-padrão são fixadas pelo 
químico, portanto de acordo com Berkson estamos no caso da observação controlada.

O modelo definido pelas equações (2.2) e (2.3) não considera erro na preparação da solução-padrão, por isso deve ser redefinido considerando as observações controladas para a variável independente. Assim, considerando as equações (2.2) e (2.3) juntamente com a equação (3.5) temos que

$$
\begin{gathered}
Y_{i}=\alpha+\beta X_{i}+\left(\epsilon_{i}-\beta \delta_{i}\right), i=1, \cdots, n, \\
Y_{0 i}=\alpha+\beta X_{0}+\epsilon_{i}, \quad i=n+1, \cdots, n+k,
\end{gathered}
$$

com as seguintes suposições sobre os erros aleatórios:

- $\epsilon_{i}$ segue uma distribuição normal com média zero e variância $\sigma_{\epsilon}^{2}$,

- $E\left(\delta_{i}\right)=0, V\left(\delta_{i}\right)=\sigma_{\delta_{i}}^{2}$,

- $\operatorname{cov}\left(\epsilon_{i}, \epsilon_{j}\right)=0, i \neq j, \operatorname{cov}\left(\delta_{i}, \delta_{j}\right)=0, i \neq j$, e $\operatorname{cov}\left(\delta_{i}, \epsilon_{j}\right)=0$ para todo $i, j$.

A variável $X$ é controlada e $\epsilon_{i}-\beta \delta_{i}$ não depende de $X_{i}$. A equação (3.6), correspondente ao primeiro estágio com $\delta_{i} \sim N\left(0, \sigma_{\delta}^{2}\right)$ é conhecida como modelo de regressão de Berkson (Cheng, 1999). Se as variâncias $\sigma_{\delta_{i}}^{2}$ são iguais para $i=1, \cdots, n$ temos o modelo de calibração homoscedástico, caso contrário, temos o modelo de calibração heteroscedástico que serão estudados a seguir.

\subsubsection{Modelo homoscedástico}

O modelo definido por (3.6) e (3.7), com a suposição adicional de que $\delta_{i}, i=1, \cdots, n$ são identicamente distribuídos com distribuição normal com média zero e variância constante $\sigma_{\delta}^{2}$, caracteriza o modelo homoscedástico.

A seguir derivamos os estimadores de máxima verossimilhança para os parâmetros desse modelo. 
Para o modelo homoscedástico, o logaritmo da função de verosimilhança é dado por:

$$
\begin{aligned}
l\left(\alpha, \beta, X_{0}, \sigma_{\epsilon}^{2}, \sigma_{\delta}^{2}\right) \propto & -\frac{n}{2} \log \left(\sigma_{\epsilon}^{2}+\beta \sigma_{\delta}^{2}\right)-\frac{k}{2} \log \left(\sigma_{\epsilon}^{2}\right)-\frac{1}{2}\left[\frac{1}{\sigma_{\epsilon}^{2}+\beta \sigma_{\delta}^{2}} \sum_{i=1}^{n}\left(Y_{i}-\alpha-\beta X_{i}\right)^{2}\right. \\
& \left.+\frac{1}{\sigma_{\epsilon}^{2}} \sum_{i=n+1}^{n+k}\left(Y_{0 i}-\alpha-\beta X_{0}\right)^{2}\right]
\end{aligned}
$$

Resolvendo $\frac{\partial l}{\partial \alpha}=0$ e $\frac{\partial l}{\partial X_{0}}=0$ obtemos os EMV de $\alpha$ e $X_{0}$, dados respectivamente por

$$
\hat{\alpha}=\bar{Y}-\hat{\beta} \bar{X} \quad \text { e } \quad \hat{X}_{0}=\frac{\bar{Y}_{0}-\hat{\alpha}}{\hat{\beta}} .
$$

Assim, a expressão (3.8) pode ser escrita como uma função apenas de $\beta, \sigma_{\epsilon}^{2}$ e $\sigma_{\delta}^{2}$, ou seja:

$$
\begin{aligned}
l\left(\beta, \sigma_{\epsilon}^{2}, \sigma_{\delta}^{2}\right) \propto & -\frac{n}{2} \log \left(\sigma_{\epsilon}^{2}+\beta \sigma_{\delta}^{2}\right)-\frac{k}{2} \log \left(\sigma_{\epsilon}^{2}\right)-\frac{1}{2}\left[\frac { 1 } { \sigma _ { \epsilon } ^ { 2 } + \beta \sigma _ { \delta } ^ { 2 } } \sum _ { i = 1 } ^ { n } \left[\left(Y_{i}-\bar{Y}\right)\right.\right. \\
& \left.\left.-\beta\left(X_{i}-\bar{X}\right)\right]^{2}+\frac{1}{\sigma_{\epsilon}^{2}} \sum_{i=n+1}^{n+k}\left(Y_{0 i}-\bar{Y}_{0}\right)^{2}\right] .
\end{aligned}
$$

Inicialmente, vamos obter, a partir de (3.9), os EMV de $\beta, \sigma_{\epsilon}^{2}$ e $\sigma_{\delta}^{2}$. A seguir vamos considerar $\sigma_{\delta}^{2}$ conhecida e obter os EMV apenas para $\beta$ e $\sigma_{\epsilon}^{2}$.

\section{Caso 1: variância $\sigma_{\delta}^{2}$ desconhecida}

Derivando a expressão (3.9) em relação aos parâmetros $\beta, \sigma_{\epsilon}^{2}$ e $\sigma_{\delta}^{2}$ e igualando a zero temos, respectivamente, as seguintes equações

$$
\begin{aligned}
\hat{\beta} \hat{\sigma}_{\delta}^{2}\left(\hat{\sigma}_{\epsilon}^{2}+\hat{\beta}^{2} \hat{\sigma}_{\delta}^{2}-S_{Y Y}+\hat{\beta} S_{X Y}\right) & =\left(S_{X Y}-\hat{\beta} S_{X X}\right) \hat{\sigma}_{\epsilon}^{2} \\
\hat{\sigma}_{\epsilon}^{2}+\hat{\beta}^{2} \hat{\sigma}_{\delta}^{2} & =S_{Y Y}-2 \hat{\beta} S_{X Y}+\hat{\beta}^{2} S_{X X} \\
\frac{k S_{Y_{0} Y_{0}}}{\left(\hat{\sigma}_{\epsilon}^{2}\right)^{2}}-\frac{k}{\hat{\sigma}_{\epsilon}^{2}} & =\frac{n}{\hat{\sigma}_{\epsilon}^{2}+\hat{\beta}^{2} \hat{\sigma}_{\delta}^{2}}-\frac{n\left(S_{Y Y}-2 \hat{\beta} S_{X Y}+\hat{\beta}^{2} S_{X X}\right)}{\left(\hat{\sigma}_{\epsilon}^{2}+\hat{\beta}^{2} \hat{\sigma}_{\delta}^{2}\right)^{2}}
\end{aligned}
$$

em que $S_{Y_{0} Y_{0}}=\frac{1}{k} \sum_{i=n+1}^{n+k}\left(Y_{0 i}-\bar{Y}_{0}\right)^{2}$.

De (3.10) e (3.11) obtemos a seguinte equação

$$
\left(\hat{\beta} S_{X X}-S_{X Y}\right)\left(S_{Y Y}-2 \hat{\beta} S_{X Y}+\hat{\beta}^{2} S_{X X}\right)=0
$$

$\log 0$

$$
\begin{aligned}
\hat{\beta} S_{X X}-S_{X Y} & =0 \text { ou } \\
S_{Y Y}-2 \hat{\beta} S_{X Y}+\hat{\beta}^{2} S_{X X} & =0 .
\end{aligned}
$$


Mas, segundo a desigualdade de Cauchy-Schwarz, $S_{X X} S_{Y Y} \geq S_{X Y}^{2}$, então a equação (3.14) tem raízes reais se e somente se $Y_{i}=c X_{i}$, em que $c$ é constante. Portanto pela equação (3.13) temos que $\hat{\beta}=\frac{S_{X Y}}{S_{X X}}$.

Isolando o estimador de $\sigma_{\delta}^{2}$ na equação (3.11) temos que

$$
\hat{\sigma}_{\delta}^{2}=\frac{\left(S_{Y Y}-2 \hat{\beta} S_{X Y}+\hat{\beta}^{2} S_{X X}\right)-\hat{\sigma}_{\epsilon}^{2}}{\hat{\beta}^{2}} .
$$

Das equações (3.11) e (3.12) obtemos o estimador de $\sigma_{\epsilon}^{2}$ dado por

$$
\hat{\sigma}_{\epsilon}^{2}=S_{Y_{0} Y_{0}}
$$

A matriz de informação esperada de Fisher de $\theta=\left(\alpha, \beta, X_{0}, \sigma_{\delta}^{2}, \sigma_{\epsilon}^{2}\right)$ é dada por:

$$
I(\theta)=\left(\begin{array}{ccccc}
\frac{n}{\gamma}+\frac{k}{\sigma_{\epsilon}^{2}} & \frac{n \bar{X}}{\gamma}+\frac{k X_{0}}{\sigma_{\epsilon}^{2}} & \frac{k \beta}{\sigma_{\epsilon}^{2}} & 0 & 0 \\
\frac{n \bar{X}}{\gamma}+\frac{k X_{0}}{\sigma_{\epsilon}^{2}} & \frac{\sum_{i=1}^{n} X_{i}^{2}}{\gamma}+\frac{2 n \beta^{2} \sigma_{\delta}^{4}}{\gamma^{2}}+\frac{k X_{0}^{2}}{\sigma_{\epsilon}^{2}} & \frac{k \beta X_{0}}{\sigma_{\epsilon}^{2}} & \frac{n \beta^{3} \sigma_{\delta}^{2}}{\gamma^{2}} & \frac{n \beta \sigma_{\delta}^{2}}{\gamma^{2}} \\
\frac{k \beta}{\sigma_{\epsilon}^{2}} & \frac{k \beta X_{0}}{\sigma_{\epsilon}^{2}} & \frac{k \beta^{2}}{\sigma_{\epsilon}^{2}} & 0 & 0 \\
0 & \frac{n \beta^{3} \sigma_{\delta}^{2}}{\gamma^{2}} & 0 & \frac{n \beta^{4}}{2 \gamma^{2}} & \frac{n \beta^{2}}{2 \gamma^{2}} \\
0 & \frac{n \beta \sigma_{\delta}^{2}}{\gamma^{2}} & 0 & \frac{n \beta^{2}}{2 \gamma^{2}} & \frac{n}{2 \gamma^{2}}+\frac{k}{2 \sigma_{\epsilon}^{4}}
\end{array}\right)
$$

em que

$$
\gamma=\beta \sigma_{\delta}^{2}+\sigma_{\epsilon}^{2}
$$

Quando $k=q n, q \in Q^{+}$e para $n \longrightarrow \infty$ o estimador $\hat{\theta}$ tem distribuição aproximadamente normal com média $\theta$ e variância $I(\theta)^{-1}$, assim temos que a variância aproximada até ordem $n^{-1}$ de $\hat{X}_{0}$ é

$$
\begin{aligned}
V_{1}\left(\hat{X}_{0}\right) & =\frac{1}{n k \beta^{2}}\left[\frac{-n \sigma_{\epsilon}^{2} \sum_{i=1}^{n} X_{i}^{2}-n k X_{0}^{2} \gamma-k \sum_{i=1}^{n} X_{i}^{2} \gamma+n^{2} \bar{X}^{2} \sigma_{\epsilon}^{2}+2 n k \bar{X} X_{0} \gamma}{-n S_{X X}}\right] \\
& =\frac{\sigma_{\epsilon}^{2}}{\beta^{2}}\left[\frac{1}{k}+\frac{\gamma}{n \sigma_{\epsilon}^{2}}+\frac{\gamma}{\sigma_{\epsilon}^{2}} \frac{\left(\bar{X}-X_{0}\right)^{2}}{n S_{X X}}\right] .
\end{aligned}
$$

Considerando $k$ fixo e expandindo $\hat{X}_{0}$ em série de Taylor no ponto $(\alpha, \beta)$ obtemos as seguintes aproximações, ignorando os termos de ordem menor que $n^{-2}$, para a variância e 
o vício de $\hat{X}_{0}$ :

$$
\begin{aligned}
V_{2}\left(\hat{X}_{0}\right) & =\frac{\sigma_{\epsilon}^{2}}{\beta^{2}}\left[\frac{1}{k}+\frac{\gamma}{n \sigma_{\epsilon}^{2}}+\frac{\gamma\left(\bar{X}-\hat{X}_{0}\right)^{2}}{\sigma_{\epsilon}^{2} n S_{X X}}+\frac{3 \gamma}{k n \beta^{2} S_{X X}}\right] \\
\operatorname{Vício}\left(\hat{X}_{0}\right) & =\frac{\gamma\left(\bar{X}-\hat{X}_{0}\right)}{\beta^{2} n S_{X X}} .
\end{aligned}
$$

Podemos observar que o estimador $\hat{X}_{0}$ de $X_{0}$ é viciado, mas é assintoticamente não viciado.

Com relação à variância do estimador $\hat{X}_{0}$, notemos que, quando $k=q n, q \in Q^{+} \mathrm{e}$ ignorando os termos de ordem menor que $n^{-1}$, a variância obtida em (3.18) coincide com a variância dada em (3.17) encontrada através da informação de Fisher. A equação (3.17) considera tamanhos de amostras grandes na primeira e segunda etapa ( $n$ e $k$ ), já a equação (3.18) considera amostra grande apenas na primeira etapa e uma amostra de tamanho fixo na segunda etapa.

Note que quando $\sigma_{\delta}^{2}=0$ as expressões das variâncias (3.17) e (3.18) são iguais a (2.10) e (2.12), respectivamente.

Para construir um intervalo de confiança para $X_{0}$ consideramos que

$$
\frac{\hat{X}_{0 C}-X_{0}}{\hat{V}\left(\hat{X}_{0}\right)} \stackrel{D}{\longrightarrow} N(0,1)
$$

em que $\hat{V}\left(\hat{X}_{C}\right)$ é a variância dada em (3.17) ou (3.18) estimada. Logo, o intervalo de confiança aproximado para $X_{0}$ com um nível de confiança igual a $(1-\alpha)$ é dado por

$$
\left(\hat{X}_{0}-z_{\frac{\alpha}{2}} \sqrt{\hat{V}\left(\hat{X}_{0}\right)}, \hat{X}_{0}+z_{\frac{\alpha}{2}} \sqrt{\hat{V}\left(\hat{X}_{0}\right)}\right)
$$

em que $z_{\frac{\alpha}{2}}$ é o quantil de ordem $\left(1-\frac{\alpha}{2}\right)$ da distribuição normal padrão.

\section{Caso 2: variância $\sigma_{\delta}^{2}$ conhecida}

Supondo agora $\sigma_{\delta}^{2}$ conhecida e igualando a zero a derivada de (3.9) em relação a $\beta$ e $\sigma_{\epsilon}^{2}$, temos as seguintes equações, respectivamente,

$$
\begin{aligned}
\hat{\beta} \sigma_{\delta}^{2}\left(\hat{\sigma}_{\epsilon}^{2}+\hat{\beta}^{2} \sigma_{\delta}^{2}-S_{Y Y}+\hat{\beta} S_{X Y}\right) & =\left(S_{X Y}-\hat{\beta} S_{X X}\right) \hat{\sigma}_{\epsilon}^{2} \\
\frac{k S_{Y_{0} Y_{0}}}{\left(\hat{\sigma}_{\epsilon}^{2}\right)^{2}}-\frac{k}{\hat{\sigma}_{\epsilon}^{2}} & =\frac{n}{\hat{\sigma}_{\epsilon}^{2}+\hat{\beta}^{2} \sigma_{\delta}^{2}}-\frac{S_{Y Y}-2 \hat{\beta} S_{X Y}+\hat{\beta}^{2} S_{X X}}{\left(\hat{\sigma}_{\epsilon}^{2}+\hat{\beta}^{2} \sigma_{\delta}^{2}\right)^{2}} .
\end{aligned}
$$


As estimativas de $\beta$ e $\sigma_{\epsilon}^{2}$ são obtidas utilizando-se algum método iterativo para resolver as equações (3.22) e (3.23).

A matriz de informação esperada de Fisher de $\theta=\left(\alpha, \beta, X_{0}, \sigma_{\epsilon}^{2}\right)$ é dada por

$$
I(\theta)=\left(\begin{array}{clrr}
\frac{n}{\gamma}+\frac{k}{\sigma_{\epsilon}^{2}} & \frac{n \bar{X}}{\gamma}+\frac{k X_{0}}{\sigma_{\epsilon}^{2}} & \frac{k \beta}{\sigma_{\epsilon}^{2}} & 0 \\
\frac{n \bar{X}}{\gamma}+\frac{k X_{0}}{\sigma_{\epsilon}^{2}} & \frac{\sum_{i=1}^{n} X_{i}^{2}}{\gamma}+\frac{2 n \beta^{2} \sigma_{\delta}^{4}}{\gamma^{2}}+\frac{k X_{0}^{2}}{\sigma_{\epsilon}^{2}} & \frac{k \beta X_{0}}{\sigma_{\epsilon}^{2}} & \frac{n \beta \sigma_{\delta}^{2}}{\gamma^{2}} \\
\frac{k \beta}{\sigma_{\epsilon}^{2}} & \frac{k \beta X_{0}}{\sigma_{\epsilon}^{2}} & \frac{k \beta^{2}}{\sigma_{\epsilon}^{2}} & 0 \\
0 & \frac{n \beta \sigma_{\delta}^{2}}{\gamma^{2}} & 0 & \frac{n}{2 \gamma^{2}}+\frac{k}{2 \sigma_{\epsilon}^{4}}
\end{array}\right)
$$

sendo o parâmetro $\gamma$ definido como em (3.16).

A variância aproximada de $\hat{X}_{0}$ obtida invertendo-se a matriz de informação de Fisher é

$$
V\left(\hat{X}_{0}\right)=\frac{\sigma_{\epsilon}^{2}}{\beta^{2}}\left[\frac{1}{k}+\frac{\gamma}{n \sigma_{\epsilon}^{2}}+\frac{\gamma}{\sigma_{\epsilon}^{2}} E\right]
$$

em que,

$$
E=\frac{n X_{0}^{2} \sigma_{\epsilon}^{4}+k X_{0}^{2} \gamma^{2}-2 n X_{0} \bar{X} \sigma_{\epsilon}^{4}-2 k X_{0} \bar{X} \gamma^{2}+n \bar{X}^{2} \sigma_{\epsilon}^{4}+k \bar{X}^{2} \gamma^{2}}{\left(n \sigma_{\epsilon}^{4}+k \gamma^{2}\right) \sum_{i=1}^{n} X_{i}^{2}+2 n k \beta^{2} \gamma \sigma_{\delta}^{4}-n^{2} \bar{X}^{2} \sigma_{\epsilon}^{4}-n k \bar{X}^{2} \gamma^{2}}
$$

Observe que fazendo $\sigma_{\delta}^{2}=0$, ou seja, a preparação da solução-padrão é isenta de erro, a expressão (3.24) se reduz a (2.10). Os EMV dos parâmetros $\alpha, \beta$ e $X_{0}$ do modelo de calibração usual são também os EMQ do modelo de calibração homoscedástico. No Apêndice 1 mostramos que sob o modelo homoscedástico esses estimadores são lineares não viciados de variância mínima. Para construir um intervalo de confiança para $X_{0}$ consideramos o intervalo (3.21), em que $\hat{V}\left(\hat{X}_{0 C}\right)$ é a variância dada em (3.24) estimada.

\subsubsection{Modelo heteroscedástico}

O modelo heteroscedástico é definido considerando o modelo dado pelas equações (3.6) e (3.7) com a suposição de que os erros $\delta_{i}, i=1, \cdots, n$ têm distribuição normal com média zero e variância $\sigma_{\delta_{i}}^{2}$, respectivamente. 


\subsection{Modelo de calibração controlada}

Note que o modelo homoscedástico é um caso particular do modelo heteroscedástico, considerando $\sigma_{\delta_{i}}^{2}=\sigma_{\delta}^{2}$ para todo $i$. Vamos considerar apenas o caso em que as variâncias $\sigma_{\delta_{i}}^{2}, i=1, \cdots, n$ são conhecidas.

Para este modelo o logaritmo da função de verossimilhança é dado por:

$$
\begin{aligned}
l\left(\alpha, \beta, X_{0}, \sigma_{\epsilon}^{2}\right) & \propto-\frac{1}{2} \sum_{i=1}^{n} \log \left(\sigma_{\epsilon}^{2}+\beta^{2} \sigma_{\delta_{i}}^{2}\right)-\frac{k}{2} \log \left(\sigma_{\epsilon}^{2}\right) \\
& -\frac{1}{2}\left[\sum_{i=1}^{n} \frac{\left(Y_{i}-\alpha-\beta X_{i}\right)^{2}}{\sigma_{\epsilon}^{2}+\beta^{2} \sigma_{\delta_{i}}^{2}}+\sum_{i=n+1}^{n+k} \frac{\left(Y_{0 i}-\alpha-\beta X_{0}\right)^{2}}{\sigma_{\epsilon}^{2}}\right] .
\end{aligned}
$$

Resolvendo $\frac{\partial L}{\partial \alpha}=0$ e $\frac{\partial L}{\partial X_{0}}=0$ obtemos os EMV de $\alpha$ e $X_{0}$ dados, respectivamente, por

$$
\hat{\alpha}=\bar{Y}-\hat{\beta} \bar{X} \quad \text { e } \quad \hat{X}_{0}=\frac{\bar{Y}_{0}-\hat{\alpha}}{\hat{\beta}} .
$$

Para obter os EMV podemos reescrever a expressão (3.25) da seguinte forma

$$
\begin{aligned}
l\left(\alpha, \beta, X_{0}, \sigma_{\epsilon}^{2}\right) \quad & \propto-\frac{1}{2} \sum_{i=1}^{n} \log \left(\sigma_{\epsilon}^{2}+\beta^{2} \sigma_{\delta_{i}}^{2}\right)-\frac{k}{2} \log \left(\sigma_{\epsilon}^{2}\right) \\
& -\frac{1}{2}\left[\sum_{i=1}^{n} \frac{\left[\left(Y_{i}-\bar{Y}\right)-\beta\left(X_{i}-\bar{X}\right)\right]^{2}}{\sigma_{\epsilon}^{2}+\beta^{2} \sigma_{\delta_{i}}^{2}}+\frac{1}{\sigma_{\epsilon}^{2}} \sum_{i=n+1}^{n+k}\left(Y_{0 i}-\bar{Y}_{0}\right)^{2}\right] .
\end{aligned}
$$

Fazendo $\frac{\partial L}{\partial \boldsymbol{\beta}}=0$ e $\frac{\partial L}{\partial \sigma_{\epsilon}^{2}}=0$, a partir dessa expressão simplificada da verossimilhança temos o seguinte sistema de equações:

$$
\begin{aligned}
\sum_{i=1}^{n} \frac{\beta \sigma_{\delta_{i}}^{2}\left[\left(\beta^{2} \sigma_{\delta_{i}}^{2}+\sigma_{\epsilon}^{2}\right)-\left(Y_{i}-\alpha-\beta X_{i}\right)^{2}\right]}{\left(\beta^{2} \sigma_{\delta_{i}}^{2}+\sigma_{\epsilon}^{2}\right)^{2}} & =\sum_{i=1}^{n} \frac{X_{i}\left(Y_{i}-\alpha-\beta X_{i}\right)}{\beta^{2} \sigma_{\delta_{i}}^{2}+\sigma_{\epsilon}^{2}} \\
\sum_{i=1}^{n} \frac{\left(\beta^{2} \sigma_{\delta_{i}}^{2}+\sigma_{\epsilon}^{2}\right)-\left(Y_{i}-\alpha-\beta X_{i}\right)^{2}}{\left(\beta^{2} \sigma_{\delta_{i}}^{2}+\sigma_{\epsilon}^{2}\right)^{2}} & =\sum_{i=n+1}^{n+k} \frac{\left(Y_{0 i}-\bar{Y}_{0}\right)^{2}}{\sigma_{\epsilon}^{4}}-\frac{k}{\sigma_{\epsilon}^{2}}
\end{aligned}
$$

As estimativas de $\beta$ e $\sigma_{\epsilon}^{2}$ podem ser obtidas com ajuda de algum método iterativo que resolva o sistema de equações (3.27) e (3.28).

A informação esperada de Fisher $I(\theta)=I\left(\alpha, \beta, X_{0}, \sigma_{\epsilon}^{2}\right)$ é dada por:

$$
I(\theta)=\left(\begin{array}{cccc}
\sum_{i=1}^{n} \frac{1}{\gamma_{i}}+\frac{k}{\sigma_{\epsilon}^{2}} & \sum_{i=1}^{n} \frac{X_{i}}{\gamma_{i}}+\frac{k X_{0}}{\sigma_{\epsilon}^{2}} & \frac{k \beta}{\sigma_{\epsilon}^{2}} & 0 \\
\sum_{i=1}^{n} \frac{X_{i}}{\gamma_{i}}+\frac{k X_{0}}{\sigma_{\epsilon}^{2}} & \sum_{i=1}^{n} \frac{X_{i}^{2}}{\gamma_{i}}+2 \beta^{2} \sum_{i=1}^{n} \frac{\sigma_{\delta_{i}}^{4}}{\gamma_{i}^{2}}+\frac{k X_{0}^{2}}{\sigma_{\epsilon}^{2}} & \frac{k \beta X_{0}}{\sigma_{\epsilon}^{2}} & \beta \sum_{i=1}^{n} \frac{\sigma_{\delta_{i}}^{2}}{\gamma_{i}^{2}} \\
\frac{k \beta}{\sigma_{\epsilon}^{2}} & \frac{k \beta X_{0}}{\sigma_{\epsilon}^{2}} & \frac{k \beta^{2}}{\sigma_{\epsilon}^{2}} & 0 \\
0 & \beta \sum_{i=1}^{n} \frac{\sigma_{\delta_{i}}^{2}}{\gamma_{i}^{2}} & 0 & \sum_{i=1}^{n} \frac{1}{2 \gamma_{i}^{2}}+\frac{k}{2 \sigma_{\epsilon}^{4}}
\end{array}\right)
$$


em que $\gamma_{i}=\beta^{2} \sigma_{\delta_{i}}^{2}+\sigma_{\epsilon}^{2}$.

A variância aproximada de $\hat{X}_{0}$ é dada por:

$$
V\left(\hat{X}_{0}\right)=\frac{\sigma_{\epsilon}^{2}}{\beta^{2}}\left[\frac{1}{n}+\frac{1}{k}-\frac{E_{1}}{n \sigma_{\epsilon}^{2} E_{2}}\right]
$$

em que

$$
\begin{aligned}
E_{1}= & -n \sum_{i=1}^{n} \frac{X_{0}^{2} \sigma_{\epsilon}^{4}}{\gamma_{i}} \sum_{i=1}^{n} \frac{1}{\gamma_{i}^{2}}-n k \sum_{i=1}^{n} \frac{X_{0}^{2}}{\gamma_{i}}-n \sum_{i=1}^{n} \frac{X_{i}^{2} \sigma_{\epsilon}^{4}}{\gamma_{i}} \sum_{i=1}^{n} \frac{1}{\gamma_{i}^{2}}-n k \sum_{i=1}^{n} \frac{X_{i}^{2}}{\gamma_{i}} \\
& -2 n \beta^{2} \sum_{i=1}^{n} \frac{\sigma_{\delta_{i}}^{4} \sigma_{\epsilon}^{4}}{\gamma_{i}^{2}} \sum_{i=1}^{n} \frac{1}{\gamma_{i}^{2}}-2 n k \beta^{2} \sum_{i=1}^{n} \frac{\sigma_{\delta_{i}}^{4}}{\gamma_{i}^{2}}+2 n \beta^{2} \sigma_{\epsilon}^{2}\left[\sum_{i=1}^{n} \frac{\sigma_{\delta_{i}}^{2}}{\gamma_{i}^{2}}\right]^{2} \\
& +2 n X_{0} \sigma_{\epsilon}^{4} \sum_{i=1}^{n} \frac{X_{i}}{\gamma_{i}} \sum_{i=1}^{n} \frac{1}{\gamma_{i}^{2}}+2 n k X_{0} \sum_{i=1}^{n} \frac{X_{i}}{\gamma_{i}}+\sigma_{\epsilon}^{6} \sum_{i=1}^{n} \frac{X_{i}^{2}}{\gamma_{i}} \sum_{i=1}^{n} \frac{1}{\gamma_{i}^{2}} \sum_{i=1}^{n} \frac{1}{\gamma_{i}} \\
& +k \sigma_{\epsilon}^{2} \sum_{i=1}^{n} \frac{X_{i}^{2}}{\gamma_{i}} \sum_{i=1}^{n} \frac{1}{\gamma_{i}}+2 \beta^{2} \sigma_{\epsilon}^{6} \sum_{i=1}^{n} \frac{\sigma_{\delta_{i}}^{4}}{\gamma_{i}^{2}} \sum_{i=1}^{n} \frac{1}{\gamma_{i}^{2}} \sum_{i=1}^{n} \frac{1}{\gamma_{i}}+2 k \beta^{2} \sigma_{\epsilon}^{2} \sum_{i=1}^{n} \frac{1}{\gamma_{i}} \sum_{i=1}^{n} \frac{\sigma_{\delta_{i}}^{4}}{\gamma_{i}^{2}} \\
& -2 \beta^{2} \sigma_{\epsilon}^{6} \sum_{i=1}^{n}\left[\frac{\sigma_{\delta_{i}}^{2}}{\gamma_{i}^{2}}\right]^{2} \sum_{i=1}^{n} \frac{1}{\gamma_{i}}-2 \sigma_{\epsilon}^{6}\left[\sum_{i=1}^{n} \frac{X_{i}}{\gamma_{i}}\right]^{2} \sum_{i=1}^{n} \frac{1}{\gamma_{i}^{2}}-k \sigma_{\epsilon}^{2}\left[\sum_{i=1}^{n} \frac{X_{i}}{\gamma_{i}}\right]^{2}
\end{aligned}
$$

$$
\begin{aligned}
E_{2}= & \sigma_{\epsilon}^{4} \sum_{i=1}^{n} \frac{X_{i}^{2}}{\gamma_{i}} \sum_{i=1}^{n} \frac{1}{\gamma_{i}^{2}} \sum_{i=1}^{n} \frac{1}{\gamma_{i}}+k \sum_{i=1}^{n} \frac{X_{i}^{2}}{\gamma_{i}} \sum_{i=1}^{n} \frac{1}{\gamma_{i}}+2 \sigma_{\epsilon}^{4} \beta^{2} \sum_{i=1}^{n} \frac{\sigma_{\delta_{i}}^{4}}{\gamma_{i}^{2}} \sum_{i=1}^{n} \frac{1}{\gamma_{i}^{2}} \sum_{i=1}^{n} \frac{1}{\gamma_{i}} \\
& +2 k \beta^{2} \sum_{i=1}^{n} \frac{\sigma_{\delta_{i}}^{4}}{\gamma_{i}^{2}} \sum_{i=1}^{n} \frac{1}{\gamma_{i}}-2 \beta^{2} \sigma_{\epsilon}^{4}\left[\sum_{i=1}^{n} \frac{\sigma_{\delta_{i}}^{2}}{\gamma_{i}^{2}}\right]^{2} \sum_{i=1}^{n} \frac{1}{\gamma_{i}}-\sigma_{\epsilon}^{4}\left[\sum_{i=1}^{n} \frac{X_{i}}{\gamma_{i}}\right]^{2} \sum_{i=1}^{n} \frac{1}{\gamma_{i}^{2}}-k\left[\sum_{i=1}^{n} \frac{X_{i}}{\gamma_{i}}\right]^{2} .
\end{aligned}
$$

e

Note que quando $\sigma_{\delta_{i}}^{2}=0, i=1, \cdots, n$, a expressão (3.29) se reduz a variância do modelo usual dada em (2.10) e quando $\sigma_{\delta_{i}}^{2}=\sigma_{\delta}^{2}$ para todo $i$, a expressão (3.29) se reduz à variância do modelo homoscedástico quando $\sigma_{\delta}^{2}$ é conhecida dada em (3.24).

Para construir um intervalo de confiança para $X_{0}$ consideramos o intervalo (3.21), em que $\hat{V}\left(\hat{X}_{0 C}\right)$ é a variância dada em (3.29) estimada. 


\subsection{Simulações}

Nesta seção apresentamos os resultados das simulações realizadas para os dois modelos homoscedásticos apresentados na Seção 3.3. O primeiro modelo supõe que a variância $\sigma_{\delta}^{2}$ é desconhecida e o segundo supõe que a variância $\sigma_{\delta}^{2}$ é conhecida, sendo nos dois casos a variância $\sigma_{\epsilon}^{2}$ considerada desconhecida.

Com o objetivo de avaliar o desempenho dos estimadores propostos e verificar o impacto de considerar erroneamente o modelo usual, foram geradas 5000 amostras do modelo homoscedástico. Os parâmetros fixados são os mesmos considerados para a simulação feita no Capítulo 2, ou seja, $\alpha=0,1, \beta=2, \sigma_{\epsilon}^{2}=0,04$ e $0,4, X_{0}=0,01,0,8$ e $1,9, X_{1}=0$ e $X_{i}=X_{i-1}+\frac{2}{n-1}, i=2, \cdots, n$. Para a variância $\sigma_{\delta}^{2}$ designamos os valores $\sigma_{\delta}^{2}=0,01$ e $\sigma_{\delta}^{2}=0,1$, denominados de variância pequena e grande, respectivamente. No primeiro estágio consideramos amostras de tamanho $n=5,20$ e 100 e no segundo estágio, $k=2,20$ e 100 .

As Tabelas 3.1, 3.2, 3.11, 3.12, 3.13 e 3.14 apresentam o vício e EQM empírico dos estimadores de $\alpha$ e $\beta$. Nas Tabelas 3.11 a 3.14 utilizamos, também, os estimadores obtidos adotando-se o modelo usual (M-usual).

As Tabelas 3.3 a 3.6 e 3.15 a 3.18 apresentam o vício e EQM empírico, a variância teórica e a média das variâncias estimadas do estimador de $X_{0}$. Além de serem considerados os resultados obtidos sob os modelos propostos nas Seções 3.3 e 3.4, apresentamos, também, os resultados obtidos adotando-se erroneamente o modelo usual. Nas Tabelas 3.3 a 3.6 são consideradas as expressões (3.17) e (3.18) obtidas para a variância de $\hat{X}_{0}$.

As Tabelas 3.7 a 3.10, 3.19 e 3.20 apresentam as porcentagens de cobertura e amplitude dos intervalos de $95 \%$ de confiança para o parâmetro $X_{0}$. Nas Tabelas 3.7 a 3.10 , as porcentagens e amplitudes $\%_{1}$ e $A_{1}$ e $\%_{2}$ e $A_{2}$ referem-se, respectivamente, aos intervalos de confiança construídos utilizando-se as expressões (3.17) e (3.18). 
As Tabelas 3.1 a 3.10 consideram o modelo homoscedástico com a variância $\sigma_{\delta}^{2}$ desconhecida.

Observando as Tabelas 3.1 e 3.2 temos que os valores do vício e EQM de $\hat{\alpha}$ e $\hat{\beta}$ independem dos valores de $X_{0}$ e $k$, isso já era esperado pois mostramos na Seção 3.3.1 que os estimadores $\hat{\alpha}$ e $\hat{\beta}$ não dependem do segundo estágio. Verificamos, também, que os valores do vício dos estimadores $\hat{\alpha}$ e $\hat{\beta}$ são pequenos independentemente do tamanho da amostra, e isso ocorre para os diferentes valores de $\sigma_{\epsilon}^{2}$ e $\sigma_{\delta}^{2}$. Em relação ao EQM, notamos que os valores dos EQM aumentam quando as variâncias de $\delta$ e $\epsilon$ aumentam, mas diminuem com o aumento do tamanho da amostra do primeiro estágio.

Analisando as Tabelas 3.3 a 3.6 temos que, quando $\sigma_{\epsilon}^{2}$ e $\sigma_{\delta}^{2}$ são pequenas (Tabela 3.3), o vício e EQM de $\hat{X}_{0}$ são pequenos mesmo para $n$ e $k$ pequenos. Notamos, também, que o acréscimo nas variâncias $\sigma_{\epsilon}^{2}$ ou $\sigma_{\delta}^{2}$ acarretam um aumento no vício e EQM empírico. Além disso, temos que o vício e o EQM de $\hat{X}_{0}$ são menores quando $X_{0}$ está no centro do intervalo de variação da variável $X$, isto é, $X_{0}=0,8$ e diminuem à medida que $n$ e $k$ crescem. Essas tabelas mostram que os valores das variâncias teóricas obtidas utilizando as expressões (3.17) e (3.18) são muito próximos independentemente dos valores de $\sigma_{\epsilon}^{2}, \sigma_{\delta}^{2}, X_{0}, n$ e $k$. Isso também se verifica em relação às médias das variâncias estimadas. Verificamos, também, que as variâncias teóricas e a média das variâncias estimadas do modelo proposto estão muito próximas dos valores de EQM quando $n \geq 20$ e $k \geq 20$. Por meio dessas tabelas, notamos que, se adotamos erroneamente os resultados obtidos sob o modelo usual, existem diferenças entre as médias das variâncias estimadas dos modelos usual e proposto. Na Tabela 3.6, verificamos que, quando $n$ é pequeno, os valores da média das variâncias estimadas do modelo proposto e do usual são muito altos.

Analisando as Tabelas 3.7 a 3.10 , observamos que para todo $n$ e $X_{0}$, se adotarmos incorretamente o modelo usual, as amplitudes diminuem muito com o aumento de $k$. Isso faz com que as porcentagens de cobertura diminuam afastando-se de $95 \%$, exceto para o caso em que $\sigma_{\delta}^{2}=0,01$ e $\sigma_{\epsilon}^{2}=0,4$ (Tabela 3.8). Já, adotando-se o modelo proposto, à 


\subsection{Simulações}

medida que $k$ cresce, as amplitudes diminuem, mas as coberturas crescem se aproximando de $95 \%$. As porcentagens de cobertura $\%_{1}$ e $\%_{2}$ e as amplitudes $A_{1}$ e $A_{2}$ são sempre muito próximas, sendo que a amplitude é menor para $X_{0}=0,8$. Em todas as tabelas, observamos que quando $k=20$ ou 100, à medida que $n$ cresce, as amplitudes dos intervalos diminuem e as coberturas se aproximam de $95 \%$. Na maioria dos casos, as amplitudes obtidas sob o modelo proposto são maiores do que as do modelo usual e estão mais próximas de $95 \%$. Para $n=5$ e $k=2$ as tabelas indicam que as porcentagens de cobertura e as amplitudes dos intervalos de confiança sob ambos modelos são bem inferiores a $95 \%$.

As Tabelas 3.11 a 3.20 apresentam os resultados para o modelo homoscedástico com a variância $\sigma_{\delta}^{2}$ conhecida. O método iterativo utilizado foi Quasi-Newton (Polak, 1997).

Nas Tabelas 3.11 a 3.14 temos que os valores do vício e EQM dos estimadores $\hat{\alpha}$ e $\hat{\beta}$ independem dos valores de $X_{0}$ e $k$ e diminuem com o aumento do tamanho da amostra do primeiro estágio, isso ocorre para os diferentes valores de $\sigma_{\epsilon}^{2}$ e $\sigma_{\delta}^{2}$. Notamos, também, que os estimadores obtidos, adotando-se o modelo usual, apresentam um acréscimo no EQM.

Analisando as Tabelas 3.15 a 3.18 temos que o vício e EQM empírico diminuem à medida que $n$ ou $k$ cresce e são menores quando $X_{0}$ está no centro do intervalo de variação de $X$, isto é, $X_{0}=0,8$. O valor do EQM empírico, adotando-se o modelo usual e o modelo proposto, são próximos aos valores teóricos da variância para todo $n, k, \sigma_{\epsilon}^{2}$ e $\sigma_{\delta}^{2}$, exceto quando $\sigma_{\delta}^{2}$ é grande e $n=5$. Quando $\sigma_{\delta}^{2}$ é pequena (Tabelas 3.15 e 3.16 ) a média das variâncias estimadas do modelo usual são próximas aos valores fornecidos pelo modelo proposto e próximas dos valores teóricos. Quando $\sigma_{\delta}^{2}$ é grande (Tabelas 3.17 e 3.18 ), em geral, a média da variância estimada do modelo usual é diferente dos resultados obtidos para o modelo proposto, sendo que a média da variância do modelo proposto é próxima da teórica.

Analisando as Tabelas 3.19 e 3.20 , notamos que valem os mesmos comentários feitos em relação às Tabelas 3.7 a 3.10 . 
Tabela 3.1: Vício e erro quadrático médio empírico de $\hat{\alpha}$ e $\hat{\beta}$, quando $\sigma_{\delta}^{2}=0,01$ e desconhecida.

\begin{tabular}{|c|c|c|c|c|c|c|c|c|c|c|}
\hline \multirow{3}{*}{$X_{0}$} & \multirow{3}{*}{$n$} & \multirow{3}{*}{$k$} & \multicolumn{4}{|c|}{$\sigma_{\epsilon}^{2}=0,04$} & \multicolumn{4}{|c|}{$\sigma_{\epsilon}^{2}=0,40$} \\
\hline & & & \multicolumn{2}{|c|}{$\hat{\alpha}$} & \multicolumn{2}{|c|}{$\hat{\beta}$} & \multicolumn{2}{|c|}{$\hat{\alpha}$} & \multicolumn{2}{|c|}{$\hat{\boldsymbol{\beta}}$} \\
\hline & & & Vício & EQM & Vício & $\mathrm{EQM}$ & Vício & EQM & Vício & EQM \\
\hline \multirow[t]{9}{*}{0,01} & \multirow[t]{3}{*}{5} & 2 & $-0,0004$ & 0,0470 & $-0,0009$ & 0,0320 & 0,0009 & 0,2610 & 0,0029 & 0,1720 \\
\hline & & 20 & 0,0004 & 0,0470 & $-0,0045$ & 0,0320 & 0,0073 & 0,2620 & $-0,0018$ & 0,1820 \\
\hline & & 100 & $-0,0059$ & 0,0470 & 0,0035 & 0,0320 & $-0,0042$ & 0,2690 & 0,0064 & 0,1840 \\
\hline & \multirow[t]{3}{*}{20} & 2 & 0,0008 & 0,0150 & $-0,0009$ & 0,0110 & 0,0025 & 0,0840 & $-0,0020$ & 0,0600 \\
\hline & & 20 & 0,0008 & 0,0150 & $-0,0016$ & 0,0110 & 0,0044 & 0,0820 & $-0,0056$ & 0,0600 \\
\hline & & 100 & 0,0012 & 0,0150 & $-0,0002$ & 0,0110 & 0,0022 & 0,0800 & 0,0020 & 0,0600 \\
\hline & \multirow[t]{3}{*}{100} & 2 & 0,0005 & 0,0032 & $-0,0003$ & 0,0024 & $-0,0001$ & 0,0170 & $-0,0004$ & 0,0130 \\
\hline & & 20 & $-0,0003$ & 0,0032 & 0,0005 & 0,0024 & 0,0022 & 0,0180 & 0,0000 & 0,0130 \\
\hline & & 100 & 0,0013 & 0,0032 & $-0,0011$ & 0,0024 & $-0,0011$ & 0,0180 & 0,0011 & 0,0130 \\
\hline \multirow[t]{9}{*}{0,8} & \multirow[t]{3}{*}{5} & 2 & $-0,0004$ & 0,0470 & $-0,0009$ & 0,0320 & 0,0009 & 0,2610 & 0,0029 & 0,1720 \\
\hline & & 20 & 0,0004 & 0,0470 & $-0,0045$ & 0,0320 & 0,0073 & 0,2620 & $-0,0018$ & 0,1820 \\
\hline & & 100 & $-0,0059$ & 0,0470 & 0,0035 & 0,0320 & $-0,0042$ & 0,2690 & 0,0064 & 0,1840 \\
\hline & \multirow[t]{3}{*}{20} & 2 & $-0,0008$ & 0,0150 & 0,0003 & 0,0110 & $-0,0070$ & 0,0830 & 0,0020 & 0,0600 \\
\hline & & 20 & $-0,0003$ & 0,0150 & 0,0001 & 0,0110 & 0,0001 & 0,0810 & $-0,0030$ & 0,0590 \\
\hline & & 100 & $-0,0010$ & 0,0150 & 0,0010 & 0,0110 & $-0,0010$ & 0,0830 & 0,0011 & 0,0570 \\
\hline & \multirow[t]{3}{*}{100} & 2 & $-0,0009$ & 0,0031 & 0,0007 & 0,0023 & 0,0005 & 0,0170 & $-0,0008$ & 0,0130 \\
\hline & & 20 & 0,0002 & 0,0031 & $-0,0008$ & 0,0023 & $-0,0033$ & 0,0170 & 0,0014 & 0,0130 \\
\hline & & 100 & $-0,0004$ & 0,0032 & 0,0002 & 0,0024 & $-0,0003$ & 0,0180 & 0,0000 & 0,0130 \\
\hline \multirow[t]{9}{*}{1,9} & \multirow[t]{3}{*}{5} & 2 & $-0,0043$ & 0,0500 & 0,0033 & 0,0330 & 0,0000 & 0,2630 & $-0,0056$ & 0,1750 \\
\hline & & 20 & $-0,0047$ & 0,0460 & 0,0002 & 0,0300 & $-0,0058$ & 0,2580 & $-0,0068$ & 0,1740 \\
\hline & & 100 & $-0,0008$ & 0,0490 & $-0,0014$ & 0,0320 & $-0,0190$ & 0,2530 & 0,0069 & 0,1800 \\
\hline & \multirow[t]{3}{*}{20} & 2 & $-0,0001$ & 0,0150 & 0,0005 & 0,0110 & $-0,0071$ & 0,0810 & 0,0069 & 0,0600 \\
\hline & & 20 & $-0,0018$ & 0,0150 & 0,0009 & 0,0110 & $-0,0022$ & 0,0820 & 0,0016 & 0,0590 \\
\hline & & 100 & 0,0015 & 0,0140 & $-0,0003$ & 0,0100 & $-0,0017$ & 0,0810 & $-0,0016$ & 0,0570 \\
\hline & \multirow[t]{3}{*}{100} & 2 & 0,0013 & 0,0031 & $-0,0013$ & 0,0023 & 0,0023 & 0,0180 & $-0,0018$ & 0,0130 \\
\hline & & 20 & $-0,0005$ & 0,0032 & 0,0003 & 0,0024 & $-0,0009$ & 0,0180 & 0,0009 & 0,0140 \\
\hline & & 100 & 0,0002 & 0,0031 & 0,0004 & 0,0023 & $-0,0012$ & 0,0180 & 0,0005 & 0,0130 \\
\hline
\end{tabular}


Tabela 3.2: Vício e erro quadrático médio empírico de $\hat{\alpha}$ e $\hat{\beta}$, quando $\sigma_{\delta}^{2}=0,1 \mathrm{e}$ desconhecida.

\begin{tabular}{|c|c|c|c|c|c|c|c|c|c|c|}
\hline \multirow{3}{*}{$X_{0}$} & \multirow{3}{*}{$n$} & \multirow{3}{*}{$k$} & \multicolumn{4}{|c|}{$\sigma_{\epsilon}^{2}=0,04$} & \multicolumn{4}{|c|}{$\sigma_{\epsilon}^{2}=0,4$} \\
\hline & & & \multicolumn{2}{|c|}{$\hat{\alpha}$} & \multicolumn{2}{|c|}{$\hat{\beta}$} & \multicolumn{2}{|c|}{$\hat{\alpha}$} & \multicolumn{2}{|c|}{$\hat{\boldsymbol{\beta}}$} \\
\hline & & & Vício & EQM & Vício & EQM & Vício & EQM & Vício & EQM \\
\hline \multirow[t]{9}{*}{0,01} & \multirow[t]{3}{*}{5} & 2 & $-0,0110$ & 0,2520 & 0,0012 & 0,1700 & $-0,0130$ & 0,4760 & 0,0190 & 0,3190 \\
\hline & & 20 & 0,0320 & 0,2650 & $-0,0200$ & 0,1770 & 0,0064 & 0,4810 & $-0,0037$ & 0,3240 \\
\hline & & 100 & 0,0140 & 0,2680 & $-0,0071$ & 0,1700 & $-0,0380$ & 0,4840 & 0,0150 & 0,3170 \\
\hline & \multirow[t]{3}{*}{20} & 2 & 0,0033 & 0,0800 & $-0,0022$ & 0,0590 & 0,0037 & 0,1560 & $-0,0052$ & 0,1130 \\
\hline & & 20 & 0,0009 & 0,0820 & $-0,0047$ & 0,0610 & 0,0024 & 0,1440 & 0,0007 & 0,1070 \\
\hline & & 100 & 0,0033 & 0,0820 & $-0,0013$ & 0,0590 & 0,0036 & 0,1470 & 0,0031 & 0,1090 \\
\hline & \multirow[t]{3}{*}{100} & 2 & 0,0012 & 0,0180 & $-0,0003$ & 0,0130 & 0,0007 & 0,0320 & $-0,0011$ & 0,0240 \\
\hline & & 20 & $-0,0021$ & 0,0170 & 0,0023 & 0,0130 & 0,0001 & 0,0310 & 0,0013 & 0,0240 \\
\hline & & 100 & 0,0024 & 0,0170 & $-0,0016$ & 0,0130 & $-0,0021$ & 0,0320 & 0,0025 & 0,0240 \\
\hline \multirow[t]{9}{*}{0,8} & \multirow[t]{3}{*}{5} & 2 & 0,0035 & 0,2600 & $-0,0030$ & 0,1790 & 0,0010 & 0,4810 & $-0,0039$ & 0,3130 \\
\hline & & 20 & $-0,0098$ & 0,2610 & 0,0034 & 0,1780 & $-0,0063$ & 0,4950 & 0,0160 & 0,3230 \\
\hline & & 100 & $-0,0046$ & 0,2630 & 0,0100 & 0,1750 & 0,0083 & 0,4820 & 0,0002 & 0,3210 \\
\hline & \multirow[t]{3}{*}{20} & 2 & $-0,0061$ & 0,0810 & 0,0031 & 0,0590 & 0,0022 & 0,1480 & $-0,0012$ & 0,1070 \\
\hline & & 20 & 0,0040 & 0,0830 & $-0,0044$ & 0,0590 & 0,0002 & 0,1490 & $-0,0034$ & 0,1050 \\
\hline & & 100 & $-0,0007$ & 0,0840 & 0,0020 & 0,0610 & 0,0036 & 0,1490 & $-0,0033$ & 0,1100 \\
\hline & \multirow[t]{3}{*}{100} & 2 & $-0,0036$ & 0,0170 & 0,0029 & 0,0130 & 0,0011 & 0,0310 & $-0,0005$ & 0,0230 \\
\hline & & 20 & 0,0003 & 0,0170 & $-0,0004$ & 0,0130 & $-0,0030$ & 0,0320 & 0,0020 & 0,0240 \\
\hline & & 100 & $-0,0019$ & 0,0170 & 0,0017 & 0,0130 & $-0,0025$ & 0,0320 & 0,0018 & 0,0240 \\
\hline \multirow[t]{9}{*}{1,9} & \multirow[t]{3}{*}{5} & 2 & 0,0015 & 0,2650 & 0,0043 & 0,1790 & 0,0041 & 0,4700 & $-0,0026$ & 0,3200 \\
\hline & & 20 & $-0,0021$ & 0,2570 & 0,0074 & 0,1720 & $-0,0160$ & 0,4820 & 0,0140 & 0,3120 \\
\hline & & 100 & 0,0008 & 0,2620 & 0,0040 & 0,1730 & $-0,0120$ & 0,4870 & 0,0140 & 0,3340 \\
\hline & \multirow[t]{3}{*}{20} & 2 & 0,0017 & 0,0800 & $-0,0008$ & 0,0590 & 0,0044 & 0,1470 & $-0,0080$ & 0,1080 \\
\hline & & 20 & 0,0026 & 0,0830 & $-0,0024$ & 0,0590 & $-0,0033$ & 0,1480 & 0,0008 & 0,1080 \\
\hline & & 100 & $-0,0049$ & 0,0820 & 0,0034 & 0,0600 & 0,0003 & 0,1490 & 0,0002 & 0,1080 \\
\hline & \multirow[t]{3}{*}{100} & 2 & 0,0019 & 0,0170 & $-0,0026$ & 0,0130 & 0,0022 & 0,0310 & $-0,0022$ & 0,0230 \\
\hline & & 20 & $-0,0024$ & 0,0180 & 0,0012 & 0,0130 & $-0,0010$ & 0,0320 & 0,0013 & 0,0240 \\
\hline & & 100 & 0,0009 & 0,0170 & 0,0009 & 0,0130 & $-0,0014$ & 0,0330 & $-0,0004$ & 0,0250 \\
\hline
\end{tabular}


Tabela 3.3: Vício e erro quadrático médio empírico, média das variâncias estimadas e variância teórica do estimador $\hat{X}_{0}$, para $\sigma_{\delta}^{2}=0,01$ e desconhecida e $\sigma_{\epsilon}^{2}=0,04$.

\begin{tabular}{|c|c|c|c|c|c|c|c|c|c|}
\hline \multirow{3}{*}{$X_{0}$} & \multirow{3}{*}{$n$} & \multirow{3}{*}{$k$} & \multirow{2}{*}{\multicolumn{2}{|c|}{ Empírico }} & \multirow{2}{*}{\multicolumn{2}{|c|}{$\begin{array}{c}\text { Teórico } \\
\text { M-proposto }\end{array}$}} & \multicolumn{3}{|c|}{ Média de $\hat{V}\left(\hat{X}_{0}\right)$} \\
\hline & & & & & & & \multirow{2}{*}{$\frac{\text { M-usual }}{\hat{V}_{1}\left(\hat{X}_{0}\right)}$} & \multicolumn{2}{|c|}{ M-proposto } \\
\hline & & & Vício & EQM & $V_{1}\left(\hat{X}_{0}\right)$ & $V_{2}\left(\hat{X}_{0}\right)$ & & $\hat{V}_{1}\left(\hat{X}_{0}\right)$ & $\hat{V}_{2}\left(\hat{X}_{0}\right)$ \\
\hline \multirow[t]{9}{*}{0,01} & 5 & 2 & $-0,0060$ & 0,0180 & 0,0170 & 0,0170 & 0,0120 & 0,0100 & 0,0100 \\
\hline & & 20 & $-0,0087$ & 0,0130 & 0,0120 & 0,0120 & 0,0072 & 0,0120 & 0,0120 \\
\hline & & 100 & $-0,0060$ & 0,0130 & 0,0120 & 0,0120 & 0,0065 & 0,0120 & 0,0120 \\
\hline & 20 & 2 & $-0,0038$ & 0,0086 & 0,0087 & 0,0087 & 0,0120 & 0,0052 & 0,0053 \\
\hline & & 20 & $-0,0028$ & 0,0043 & 0,0042 & 0,0042 & 0,0033 & 0,0040 & 0,0040 \\
\hline & & 100 & $-0,0032$ & 0,0038 & 0,0038 & 0,0038 & 0,0022 & 0,0036 & 0,0036 \\
\hline & 100 & 2 & $-0,0023$ & 0,0058 & 0,0058 & 0,0058 & 0,0100 & 0,0027 & 0,0027 \\
\hline & & 20 & $-0,0002$ & 0,0013 & 0,0013 & 0,0013 & 0,0016 & 0,0012 & 0,0012 \\
\hline & & 100 & $-0,0007$ & 0,0008 & 0,0009 & 0,0009 & 0,0007 & 0,0009 & 0,0009 \\
\hline \multirow[t]{9}{*}{0,8} & 5 & 2 & $-0,0011$ & 0,0094 & 0,0093 & 0,0094 & 0,0079 & 0,0045 & 0,0046 \\
\hline & & 20 & $-0,0034$ & 0,0050 & 0,0048 & 0,0048 & 0,0029 & 0,0045 & 0,0046 \\
\hline & & 100 & $-0,0007$ & 0,0047 & 0,0044 & 0,0044 & 0,0024 & 0,0045 & 0,0045 \\
\hline & 20 & 2 & 0,0005 & 0,0063 & 0,0061 & 0,0061 & 0,0095 & 0,0028 & 0,0029 \\
\hline & & 20 & 0,0007 & 0,0016 & 0,0016 & 0,0016 & 0,0015 & 0,0015 & 0,0015 \\
\hline & & 100 & $-0,0001$ & 0,0012 & 0,0012 & 0,0012 & 0,0008 & 0,0012 & 0,0012 \\
\hline & 100 & 2 & 0,0005 & 0,0050 & 0,0052 & 0,0052 & 0,0099 & 0,0021 & 0,0021 \\
\hline & & 20 & $-0,0001$ & 0,0007 & 0,0007 & 0,0007 & 0,0011 & 0,0007 & 0,0007 \\
\hline & & 100 & $-0,0003$ & 0,0003 & 0,0003 & 0,0003 & 0,0003 & 0,0003 & 0,0003 \\
\hline \multirow[t]{9}{*}{1,9} & 5 & 2 & 0,0041 & 0,0160 & 0,0150 & 0,0160 & 0,0120 & 0,0093 & 0,0094 \\
\hline & & 20 & 0,0026 & 0,0110 & 0,0110 & 0,0110 & 0,0065 & 0,0110 & 0,0110 \\
\hline & & 100 & 0,0076 & 0,0110 & 0,0110 & 0,0110 & 0,0058 & 0,0110 & 0,0110 \\
\hline & 20 & 2 & 0,0006 & 0,0079 & 0,0082 & 0,0082 & 0,0110 & 0,0049 & 0,0049 \\
\hline & & 20 & 0,0040 & 0,0039 & 0,0037 & 0,0037 & 0,0030 & 0,0035 & 0,0035 \\
\hline & & 100 & 0,0008 & 0,0033 & 0,0033 & 0,0033 & 0,0019 & 0,0031 & 0,0031 \\
\hline & 100 & 2 & 0,0020 & 0,0057 & 0,0057 & 0,0057 & 0,0100 & 0,0025 & 0,0025 \\
\hline & & 20 & 0,0003 & 0,0012 & 0,0012 & 0,0012 & 0,0015 & 0,0011 & 0,0011 \\
\hline & & 100 & 0,0003 & 0,0008 & 0,0008 & 0,0008 & 0,0006 & 0,0008 & 0,0008 \\
\hline
\end{tabular}


Tabela 3.4: Vício e erro quadrático médio empírico, média das variâncias estimadas e variância teórica do estimador $\hat{X}_{0}$, para $\sigma_{\delta}^{2}=0,01$ e desconhecida e $\sigma_{\epsilon}^{2}=0,4$.

\begin{tabular}{|c|c|c|c|c|c|c|c|c|c|}
\hline \multirow{3}{*}{$X_{0}$} & \multirow{3}{*}{$n$} & \multirow{3}{*}{$k$} & \multirow{2}{*}{\multicolumn{2}{|c|}{ Empírico }} & \multirow{2}{*}{\multicolumn{2}{|c|}{$\begin{array}{c}\text { Teórico } \\
\text { M-proposto }\end{array}$}} & \multicolumn{3}{|c|}{ Média de $\hat{V}\left(\hat{X}_{0}\right)$} \\
\hline & & & & & & & \multirow{2}{*}{$\frac{\text { M-usual }}{\hat{V}_{1}\left(\hat{X}_{0}\right)}$} & \multicolumn{2}{|c|}{ M-proposto } \\
\hline & & & Vício & EQM & $V_{1}\left(\hat{X}_{0}\right)$ & $V_{2}\left(\hat{X}_{0}\right)$ & & $\hat{V}_{1}\left(\hat{X}_{0}\right)$ & $\hat{V}_{2}\left(\hat{X}_{0}\right)$ \\
\hline \multirow[t]{9}{*}{0,01} & \multirow[t]{3}{*}{5} & 2 & $-0,0550$ & 0,1550 & 0,1150 & 0,1220 & 0,0980 & 0,0900 & 0,0920 \\
\hline & & 20 & $-0,0630$ & 0,1060 & 0,0700 & 0,0710 & 0,0990 & 0,1410 & 0,1420 \\
\hline & & 100 & $-0,0570$ & 0,1020 & 0,0660 & 0,0660 & 0,1030 & 0,1510 & 0,1510 \\
\hline & \multirow[t]{3}{*}{20} & 2 & $-0,0084$ & 0,0740 & 0,0700 & 0,0720 & 0,0700 & 0,0350 & 0,0350 \\
\hline & & 20 & $-0,0180$ & 0,0280 & 0,0250 & 0,0250 & 0,0260 & 0,0290 & 0,0290 \\
\hline & & 100 & $-0,0130$ & 0,0230 & 0,0210 & 0,0210 & 0,0220 & 0,0270 & 0,0270 \\
\hline & \multirow[t]{3}{*}{100} & 2 & $-0,0066$ & 0,0560 & 0,0540 & 0,0550 & 0,0590 & 0,0180 & 0,0180 \\
\hline & & 20 & $-0,0027$ & 0,0093 & 0,0093 & 0,0093 & 0,0096 & 0,0085 & 0,0085 \\
\hline & & 100 & $-0,0042$ & 0,0051 & 0,0053 & 0,0053 & 0,0052 & 0,0055 & 0,0055 \\
\hline \multirow[t]{9}{*}{0,8} & \multirow[t]{3}{*}{5} & 2 & $-0,0130$ & 0,0880 & 0,0740 & 0,0800 & 0,0560 & 0,0360 & 0,0380 \\
\hline & & 20 & $-0,0150$ & 0,0340 & 0,0290 & 0,0290 & 0,0320 & 0,0430 & 0,0440 \\
\hline & & 100 & $-0,0061$ & 0,0310 & 0,0250 & 0,0250 & 0,0300 & 0,0430 & 0,0430 \\
\hline & \multirow[t]{3}{*}{20} & 2 & 0,0006 & 0,0580 & 0,0560 & 0,0580 & 0,0560 & 0,0200 & 0,0200 \\
\hline & & 20 & $-0,0014$ & 0,0110 & 0,0110 & 0,0110 & 0,0110 & 0,0110 & 0,0110 \\
\hline & & 100 & $-0,0066$ & 0,0076 & 0,0071 & 0,0071 & 0,0071 & 0,0086 & 0,0087 \\
\hline & \multirow[t]{3}{*}{100} & 2 & $-0,0004$ & 0,0520 & 0,0510 & 0,0520 & 0,0550 & 0,0150 & 0,0150 \\
\hline & & 20 & $-0,0019$ & 0,0063 & 0,0062 & 0,0063 & 0,0066 & 0,0053 & 0,0053 \\
\hline & & 100 & 0,0002 & 0,0024 & 0,0022 & 0,0022 & 0,0022 & 0,0022 & 0,0022 \\
\hline \multirow[t]{9}{*}{1,9} & \multirow[t]{3}{*}{5} & 2 & 0,0440 & 0,1400 & 0,1080 & 0,1140 & 0,0880 & 0,0780 & 0,0800 \\
\hline & & 20 & 0,0430 & 0,0840 & 0,0630 & 0,0630 & 0,0800 & 0,1130 & 0,1140 \\
\hline & & 100 & 0,0550 & 0,0880 & 0,0590 & 0,0590 & 0,0850 & 0,1290 & 0,1290 \\
\hline & \multirow[t]{3}{*}{20} & 2 & 0,0110 & 0,0720 & 0,0680 & 0,0700 & 0,0680 & 0,0320 & 0,0330 \\
\hline & & 20 & 0,0160 & 0,0250 & 0,0230 & 0,0230 & 0,0230 & 0,0260 & 0,0260 \\
\hline & & 100 & 0,0160 & 0,0210 & 0,0190 & 0,0190 & 0,0190 & 0,0240 & 0,0240 \\
\hline & \multirow[t]{3}{*}{100} & 2 & 0,0057 & 0,0560 & 0,0540 & 0,0540 & 0,0580 & 0,0170 & 0,0170 \\
\hline & & 20 & 0,0022 & 0,0088 & 0,0087 & 0,0088 & 0,0090 & 0,0079 & 0,0079 \\
\hline & & 100 & 0,0017 & 0,0049 & 0,0047 & 0,0047 & 0,0046 & 0,0049 & 0,0049 \\
\hline
\end{tabular}


Tabela 3.5: Vício e erro quadrático médio empírico, média das variâncias estimadas e variância teórica do estimador $\hat{X}_{0}$, para $\sigma_{\delta}^{2}=0,1$ e desconhecida e $\sigma_{\epsilon}^{2}=0,04$.

\begin{tabular}{|c|c|c|c|c|c|c|c|c|c|}
\hline \multirow{3}{*}{$X_{0}$} & \multirow{3}{*}{$n$} & \multirow{3}{*}{$k$} & \multirow{2}{*}{\multicolumn{2}{|c|}{ Empírico }} & \multirow{2}{*}{\multicolumn{2}{|c|}{$\frac{\text { Teórico }}{\text { M-proposto }}$}} & \multicolumn{3}{|c|}{ Média de $\hat{V}\left(\hat{X}_{0}\right)$} \\
\hline & & & & & & & \multirow{2}{*}{$\begin{array}{c}\text { M-usual } \\
\hat{V}_{1}\left(\hat{X}_{0}\right) \\
\end{array}$} & \multicolumn{2}{|c|}{ M-proposto } \\
\hline & & & Vício & EQM & $V_{1}\left(\hat{X}_{0}\right)$ & $V_{2}\left(\hat{X}_{0}\right)$ & & $\hat{V}_{1}\left(\hat{X}_{0}\right)$ & $\hat{V}_{2}\left(\hat{X}_{0}\right)$ \\
\hline \multirow[t]{9}{*}{0,01} & \multirow[t]{3}{*}{5} & 2 & $-0,0510$ & 0,1000 & 0,0700 & 0,0710 & 0,0770 & 0,0680 & 0,0690 \\
\hline & & 20 & $-0,0500$ & 0,0950 & 0,0660 & 0,0660 & 0,0220 & 0,0660 & 0,0660 \\
\hline & & 100 & $-0,0510$ & 0,0950 & 0,0650 & 0,0650 & 0,0130 & 0,0730 & 0,0730 \\
\hline & \multirow[t]{3}{*}{20} & 2 & $-0,0180$ & 0,0280 & 0,0250 & 0,0250 & 0,0670 & 0,0240 & 0,0240 \\
\hline & & 20 & $-0,0160$ & 0,0230 & 0,0210 & 0,0210 & 0,0140 & 0,0210 & 0,0210 \\
\hline & & 100 & $-0,0170$ & 0,0230 & 0,0200 & 0,0200 & 0,0055 & 0,0210 & 0,0210 \\
\hline & \multirow[t]{3}{*}{100} & 2 & $-0,0046$ & 0,0094 & 0,0093 & 0,0093 & 0,0580 & 0,0069 & 0,0069 \\
\hline & & 20 & $-0,0026$ & 0,0048 & 0,0048 & 0,0048 & 0,0082 & 0,0048 & 0,0048 \\
\hline & & 100 & $-0,0033$ & 0,0043 & 0,0044 & 0,0044 & 0,0029 & 0,0044 & 0,0044 \\
\hline \multirow[t]{9}{*}{0,8} & \multirow[t]{3}{*}{5} & 2 & $-0,0084$ & 0,0370 & 0,0290 & 0,0290 & 0,0460 & 0,0250 & 0,0250 \\
\hline & & 20 & $-0,0095$ & 0,0270 & 0,0240 & 0,0240 & 0,0071 & 0,0200 & 0,0200 \\
\hline & & 100 & $-0,0072$ & 0,0290 & 0,0240 & 0,0240 & 0,0037 & 0,0200 & 0,0200 \\
\hline & \multirow[t]{3}{*}{20} & 2 & $-0,0030$ & 0,0120 & 0,0110 & 0,0110 & 0,0530 & 0,0085 & 0,0086 \\
\hline & & 20 & $-0,0040$ & 0,0068 & 0,0066 & 0,0066 & 0,0061 & 0,0064 & 0,0064 \\
\hline & & 100 & $-0,0031$ & 0,0063 & 0,0062 & 0,0062 & 0,0017 & 0,0060 & 0,0060 \\
\hline & \multirow[t]{3}{*}{100} & 2 & $-0,0011$ & 0,0063 & 0,0062 & 0,0063 & 0,0550 & 0,0038 & 0,0038 \\
\hline & & 20 & $-0,0015$ & 0,0017 & 0,0017 & 0,0017 & 0,0057 & 0,0017 & 0,0017 \\
\hline & & 100 & $-0,0011$ & 0,0014 & 0,0013 & 0,0013 & 0,0013 & 0,0013 & 0,0013 \\
\hline \multirow[t]{9}{*}{1,9} & \multirow[t]{3}{*}{5} & 2 & 0,0430 & 0,1090 & 0,0630 & 0,0630 & 0,0830 & 0,0750 & 0,0750 \\
\hline & & 20 & 0,0450 & 0,0860 & 0,0580 & 0,0580 & 0,0210 & 0,0650 & 0,0650 \\
\hline & & 100 & 0,0410 & 0,0860 & 0,0580 & 0,0580 & 0,0110 & 0,0600 & 0,0600 \\
\hline & \multirow[t]{3}{*}{20} & 2 & 0,0160 & 0,0260 & 0,0230 & 0,0230 & 0,0650 & 0,0210 & 0,0210 \\
\hline & & 20 & 0,0140 & 0,0190 & 0,0180 & 0,0180 & 0,0130 & 0,0180 & 0,0180 \\
\hline & & 100 & 0,0170 & 0,0200 & 0,0180 & 0,0180 & 0,0048 & 0,0180 & 0,0180 \\
\hline & \multirow[t]{3}{*}{100} & 2 & 0,0050 & 0,0088 & 0,0087 & 0,0088 & 0,0570 & 0,0063 & 0,0063 \\
\hline & & 20 & 0,0030 & 0,0043 & 0,0042 & 0,0042 & 0,0078 & 0,0042 & 0,0042 \\
\hline & & 100 & 0,0020 & 0,0039 & 0,0038 & 0,0038 & 0,0026 & 0,0038 & 0,0038 \\
\hline
\end{tabular}


Tabela 3.6: Vício e erro quadrático médio empírico, média das variâncias estimadas e variância teórica do estimador $\hat{X}_{0}$, para $\sigma_{\delta}^{2}=0,1$ e desconhecida e $\sigma_{\epsilon}^{2}=0,4$.

\begin{tabular}{|c|c|c|c|c|c|c|c|c|c|}
\hline \multirow{3}{*}{$X_{0}$} & \multirow{3}{*}{$n$} & \multirow{3}{*}{$k$} & \multirow{2}{*}{\multicolumn{2}{|c|}{ Empírico }} & \multirow{2}{*}{\multicolumn{2}{|c|}{$\frac{\text { Teórico }}{\text { M-proposto }}$}} & \multicolumn{3}{|c|}{ Média de $\hat{V}\left(\hat{X}_{0}\right)$} \\
\hline & & & & & & & \multirow{2}{*}{$\begin{array}{c}\text { M-usual } \\
\hat{V}_{1}\left(\hat{X}_{0}\right) \\
\end{array}$} & \multicolumn{2}{|c|}{ M-proposto } \\
\hline & & & Vício & EQM & $V_{1}\left(\hat{X}_{0}\right)$ & $V_{2}\left(\hat{X}_{0}\right)$ & & $\hat{V}_{1}\left(\hat{X}_{0}\right)$ & $\hat{V}_{2}\left(\hat{X}_{0}\right)$ \\
\hline \multirow[t]{9}{*}{0,01} & \multirow[t]{3}{*}{5} & 2 & $-0,1610$ & 7,8790 & 0,1680 & 0,1800 & 653,0490 & 836,7600 & 839,6970 \\
\hline & & 20 & $-0,1430$ & 0,9220 & 0,1230 & 0,1250 & 16,3500 & 43,9950 & 44,2240 \\
\hline & & 100 & $-0,1040$ & 0,5110 & 0,1190 & 0,1200 & 14,3200 & 48,2670 & 48,5160 \\
\hline & \multirow[t]{3}{*}{20} & 2 & $-0,0230$ & 0,1010 & 0,0870 & 0,0910 & 0,1340 & 0,0640 & 0,0660 \\
\hline & & 20 & $-0,0310$ & 0,0530 & 0,0420 & 0,0420 & 0,0420 & 0,0510 & 0,0510 \\
\hline & & 100 & $-0,0260$ & 0,0450 & 0,0380 & 0,0380 & 0,0280 & 0,0450 & 0,0450 \\
\hline & \multirow[t]{3}{*}{100} & 2 & $-0,0110$ & 0,0600 & 0,0580 & 0,0590 & 0,1080 & 0,0280 & 0,0290 \\
\hline & & 20 & $-0,0037$ & 0,0130 & 0,0130 & 0,0130 & 0,0160 & 0,0130 & 0,0130 \\
\hline & & 100 & $-0,0056$ & 0,0090 & 0,0088 & 0,0088 & 0,0075 & 0,0090 & 0,0090 \\
\hline \multirow[t]{9}{*}{0,8} & \multirow[t]{3}{*}{5} & 2 & $-0,0260$ & 0,2290 & 0,0930 & 0,1050 & 4,4400 & 5,5290 & 7,9950 \\
\hline & & 20 & $-0,0380$ & 0,0990 & 0,0480 & 0,0490 & 0,3110 & 0,4490 & 0,5670 \\
\hline & & 100 & $-0,0170$ & 0,0760 & 0,0440 & 0,0440 & 0,1830 & 0,2500 & 0,2560 \\
\hline & \multirow[t]{3}{*}{20} & 2 & $-0,0054$ & 0,0670 & 0,0610 & 0,0650 & 0,1070 & 0,0340 & 0,0360 \\
\hline & & 20 & $-0,0064$ & 0,0180 & 0,0160 & 0,0160 & 0,0170 & 0,0170 & 0,0180 \\
\hline & & 100 & $-0,0089$ & 0,0130 & 0,0120 & 0,0120 & 0,0086 & 0,0130 & 0,0130 \\
\hline & \multirow[t]{3}{*}{100} & 2 & $-0,0041$ & 0,0510 & 0,0520 & 0,0530 & 0,1010 & 0,0220 & 0,0230 \\
\hline & & 20 & $-0,0006$ & 0,0069 & 0,0072 & 0,0073 & 0,0110 & 0,0071 & 0,0071 \\
\hline & & 100 & $-0,0012$ & 0,0033 & 0,0032 & 0,0033 & 0,0032 & 0,0033 & 0,0033 \\
\hline \multirow[t]{9}{*}{1,9} & \multirow[t]{3}{*}{5} & 2 & 0,0970 & 0,3220 & 0,1550 & 0,1670 & 0,4400 & 0,5060 & 0,5210 \\
\hline & & 20 & 0,1020 & 0,3300 & 0,1100 & 0,1110 & 0,9560 & 1,6610 & 1,6850 \\
\hline & & 100 & 0,1040 & 0,3080 & 0,1060 & 0,1060 & 0,4550 & 0,8030 & 0,8060 \\
\hline & \multirow[t]{3}{*}{20} & 2 & 0,0260 & 0,0950 & 0,0820 & 0,0860 & 0,1290 & 0,0600 & 0,0620 \\
\hline & & 20 & 0,0290 & 0,0450 & 0,0370 & 0,0370 & 0,0370 & 0,0450 & 0,0450 \\
\hline & & 100 & 0,0260 & 0,0390 & 0,0330 & 0,0330 & 0,0250 & 0,0400 & 0,0400 \\
\hline & \multirow[t]{3}{*}{100} & 2 & 0,0094 & 0,0590 & 0,0570 & 0,0580 & 0,1060 & 0,0270 & 0,0270 \\
\hline & & 20 & 0,0043 & 0,0120 & 0,0120 & 0,0120 & 0,0150 & 0,0120 & 0,0120 \\
\hline & & 100 & 0,0039 & 0,0080 & 0,0078 & 0,0078 & 0,0067 & 0,0079 & 0,0080 \\
\hline
\end{tabular}


Tabela 3.7: Porcentagem de cobertura (\%) e amplitude (A) dos intervalos de $95 \%$ de confiança para o parâmetro $X_{0}$, quando $\sigma_{\delta}^{2}=0,01$ e desconhecida e $\sigma_{\epsilon}^{2}=0,04$.

\begin{tabular}{|c|c|c|c|c|c|c|c|c|}
\hline \multirow[t]{2}{*}{$X_{0}$} & \multirow[t]{2}{*}{$n$} & \multirow[t]{2}{*}{$k$} & \multicolumn{2}{|c|}{ M-usual } & \multicolumn{4}{|c|}{ M-proposto } \\
\hline & & & $\%$ & A & $\% 1$ & $A_{1}$ & $\%_{2}$ & $\mathrm{~A}_{2}$ \\
\hline \multirow[t]{9}{*}{0,01} & \multirow[t]{3}{*}{5} & 2 & 83,04 & 0,40 & 79,34 & 0,36 & 79,37 & 0,36 \\
\hline & & 20 & 84,95 & 0,32 & 91,15 & 0,41 & 91,15 & 0,41 \\
\hline & & 100 & 83,72 & 0,31 & 92,24 & 0,42 & 92,24 & 0,42 \\
\hline & \multirow[t]{3}{*}{20} & 2 & 96,46 & 0,42 & 83,48 & 0,28 & 83,52 & 0,28 \\
\hline & & 20 & 90,19 & 0,22 & 92,47 & 0,24 & 92,47 & 0,24 \\
\hline & & 100 & 86,16 & 0,18 & 92,78 & 0,23 & 92,78 & 0,23 \\
\hline & \multirow[t]{3}{*}{100} & 2 & 98,84 & 0,40 & 73,16 & 0,19 & 73,16 & 0,19 \\
\hline & & 20 & 96,71 & 0,16 & 94,14 & 0,14 & 94,14 & 0,14 \\
\hline & & 100 & 91,90 & 0,11 & 94,68 & 0,12 & 94,68 & 0,12 \\
\hline \multirow[t]{9}{*}{0,8} & \multirow[t]{3}{*}{5} & 2 & 85,43 & 0,32 & 74,33 & 0,24 & 74,39 & 0,24 \\
\hline & & 20 & 85,16 & 0,21 & 91,34 & 0,26 & 91,34 & 0,26 \\
\hline & & 100 & 85,04 & 0,19 & 92,50 & 0,25 & 92,50 & 0,25 \\
\hline & \multirow[t]{3}{*}{20} & 2 & 97,90 & 0,38 & 73,55 & 0,20 & 73,55 & 0,20 \\
\hline & & 20 & 93,55 & 0,15 & 93,89 & 0,15 & 93,89 & 0,15 \\
\hline & & 100 & 86,53 & 0,11 & 93,12 & 0,13 & 93,12 & 0,13 \\
\hline & \multirow[t]{3}{*}{100} & 2 & 99,41 & 0,39 & 65,05 & 0,16 & 65,05 & 0,16 \\
\hline & & 20 & 98,54 & 0,13 & 94,05 & 0,10 & 94,05 & 0,10 \\
\hline & & 100 & 94,56 & 0,07 & 94,86 & 0,07 & 94,86 & 0,07 \\
\hline \multirow[t]{9}{*}{1,9} & \multirow[t]{3}{*}{5} & 2 & 82,47 & 0,39 & 78,05 & 0,35 & 78,13 & 0,35 \\
\hline & & 20 & 84,50 & 0,31 & 90,92 & 0,39 & 90,92 & 0,39 \\
\hline & & 100 & 84,75 & 0,29 & 92,83 & 0,39 & 92,83 & 0,39 \\
\hline & \multirow[t]{3}{*}{20} & 2 & 96,79 & 0,41 & 83,09 & 0,26 & 83,11 & 0,26 \\
\hline & & 20 & 91,32 & 0,21 & 93,29 & 0,23 & 93,31 & 0,23 \\
\hline & & 100 & 86,43 & 0,17 & 93,32 & 0,22 & 93,32 & 0,22 \\
\hline & \multirow[t]{3}{*}{100} & 2 & 98,88 & 0,40 & 73,06 & 0,19 & 73,06 & 0,19 \\
\hline & & 20 & 97,12 & 0,15 & 94,31 & 0,13 & 94,31 & 0,13 \\
\hline & & 100 & 92,56 & 0,10 & 94,74 & 0,11 & 94,74 & 0,11 \\
\hline
\end{tabular}


Tabela 3.8: Porcentagem de cobertura (\%) e amplitude (A) dos intervalos de $95 \%$ de confiança para o parâmetro $X_{0}$, quando $\sigma_{\delta}^{2}=0,01$ e desconhecida e $\sigma_{\epsilon}^{2}=0,4$.

\begin{tabular}{|c|c|c|c|c|c|c|c|c|}
\hline \multirow[t]{2}{*}{$X_{0}$} & \multirow[t]{2}{*}{$n$} & \multirow[t]{2}{*}{$k$} & \multicolumn{2}{|c|}{ M-usual } & \multicolumn{4}{|c|}{ M-proposto } \\
\hline & & & $\%$ & A & $\% 1$ & $\mathrm{~A}_{1}$ & $\% 2$ & $\mathrm{~A}_{2}$ \\
\hline \multirow[t]{9}{*}{0,01} & 5 & 2 & 81,85 & 1,06 & 78,68 & 0,99 & 78,95 & 1,00 \\
\hline & & 20 & 92,17 & 1,07 & 94,61 & 1,26 & 94,76 & 1,27 \\
\hline & & 100 & 93,80 & 1,11 & 96,36 & 1,33 & 96,36 & 1,33 \\
\hline & 20 & 2 & 92,72 & 1,02 & 77,52 & 0,70 & 77,84 & 0,71 \\
\hline & & 20 & 92,92 & 0,61 & 94,01 & 0,64 & 94,08 & 0,64 \\
\hline & & 100 & 93,61 & 0,57 & 95,42 & 0,63 & 95,42 & 0,63 \\
\hline & 100 & 2 & 95,46 & 0,94 & 65,12 & 0,49 & 65,14 & 0,49 \\
\hline & & 20 & 95,17 & 0,38 & 93,98 & 0,36 & 94,04 & 0,36 \\
\hline & & 100 & 94,37 & 0,28 & 94,89 & 0,29 & 94,95 & 0,29 \\
\hline \multirow[t]{9}{*}{0,8} & 5 & 2 & 81,57 & 0,84 & 71,76 & 0,66 & 72,18 & 0,68 \\
\hline & & 20 & 92,43 & 0,64 & 95,47 & 0,75 & 95,71 & 0,75 \\
\hline & & 100 & 94,19 & 0,63 & 97,37 & 0,76 & 97,37 & 0,76 \\
\hline & 20 & 2 & 93,61 & 0,91 & 68,63 & 0,52 & 69,18 & 0,52 \\
\hline & & 20 & 94,44 & 0,41 & 94,67 & 0,41 & 94,96 & 0,41 \\
\hline & & 100 & 95,13 & 0,32 & 96,89 & 0,35 & 96,94 & 0,36 \\
\hline & 100 & 2 & 95,75 & 0,92 & 59,96 & 0,42 & 60,08 & 0,42 \\
\hline & & 20 & 95,28 & 0,32 & 92,36 & 0,28 & 92,39 & 0,28 \\
\hline & & 100 & 94,55 & 0,19 & 94,64 & 0,19 & 94,67 & 0,19 \\
\hline \multirow[t]{9}{*}{1,9} & 5 & 2 & 81,81 & 1,04 & 78,22 & 0,95 & 78,43 & 0,96 \\
\hline & & 20 & 92,86 & 1,01 & 95,52 & 1,18 & 95,59 & 1,19 \\
\hline & & 100 & 92,77 & 1,02 & 95,50 & 1,23 & 95,50 & 1,23 \\
\hline & 20 & 2 & 93,54 & 1,00 & 78,57 & 0,67 & 78,77 & 0,68 \\
\hline & & 20 & 93,13 & 0,58 & 93,98 & 0,61 & 94,13 & 0,61 \\
\hline & & 100 & 93,38 & 0,53 & 95,10 & 0,58 & 95,10 & 0,59 \\
\hline & 100 & 2 & 95,24 & 0,94 & 65,27 & 0,48 & 65,39 & 0,48 \\
\hline & & 20 & 94,98 & 0,37 & 93,31 & 0,35 & 93,43 & 0,35 \\
\hline & & 100 & 94,55 & 0,27 & 95,06 & 0,27 & 95,06 & 0,27 \\
\hline
\end{tabular}


Tabela 3.9: Porcentagem de cobertura (\%) e amplitude (A) dos intervalos de $95 \%$ de confiança para o parâmetro $X_{0}$, quando $\sigma_{\delta}^{2}=0,1$ e desconhecida e $\sigma_{\epsilon}^{2}=0,04$.

\begin{tabular}{|c|c|c|c|c|c|c|c|c|}
\hline \multirow[t]{2}{*}{$X_{0}$} & \multirow[t]{2}{*}{$n$} & \multirow[t]{2}{*}{$k$} & \multicolumn{2}{|c|}{ M-usual } & \multicolumn{4}{|c|}{ M-proposto } \\
\hline & & & $\%$ & $\mathrm{~A}$ & $\% 1$ & $\mathrm{~A}_{1}$ & $\%_{2}$ & $\mathrm{~A}_{2}$ \\
\hline \multirow[t]{9}{*}{0,01} & \multirow[t]{3}{*}{5} & 2 & 84,89 & 0,94 & 80,73 & 0,85 & 80,79 & 0,86 \\
\hline & & 20 & 64,10 & 0,51 & 82,75 & 0,86 & 82,75 & 0,86 \\
\hline & & 100 & 52,09 & 0,39 & 82,42 & 0,86 & 82,42 & 0,86 \\
\hline & \multirow[t]{3}{*}{20} & 2 & 99,40 & 0,99 & 91,04 & 0,58 & 91,10 & 0,58 \\
\hline & & 20 & 87,10 & 0,45 & 92,60 & 0,55 & 92,62 & 0,55 \\
\hline & & 100 & 65,70 & 0,28 & 92,16 & 0,55 & 92,18 & 0,55 \\
\hline & \multirow[t]{3}{*}{100} & 2 & 100,00 & 0,94 & 87,60 & 0,32 & 87,66 & 0,32 \\
\hline & & 20 & 98,82 & 0,36 & 94,88 & 0,27 & 94,88 & 0,27 \\
\hline & & 100 & 89,12 & 0,21 & 94,84 & 0,26 & 94,84 & 0,26 \\
\hline \multirow[t]{9}{*}{0,8} & \multirow[t]{3}{*}{5} & 2 & 90,27 & 0,73 & 80,63 & 0,52 & 80,75 & 0,52 \\
\hline & & 20 & 64,39 & 0,31 & 82,68 & 0,51 & 82,74 & 0,51 \\
\hline & & 100 & 50,57 & 0,23 & 84,08 & 0,50 & 84,08 & 0,50 \\
\hline & \multirow[t]{3}{*}{20} & 2 & 99,92 & 0,88 & 87,30 & 0,35 & 87,50 & 0,35 \\
\hline & & 20 & 91,80 & 0,30 & 92,78 & 0,31 & 92,82 & 0,31 \\
\hline & & 100 & 67,46 & 0,16 & 92,38 & 0,30 & 92,38 & 0,30 \\
\hline & \multirow[t]{3}{*}{100} & 2 & 100,00 & 0,91 & 77,88 & 0,22 & 77,98 & 0,22 \\
\hline & & 20 & 99,94 & 0,29 & 94,98 & 0,16 & 95,04 & 0,16 \\
\hline & & 100 & 94,60 & 0,14 & 94,92 & 0,14 & 94,92 & 0,14 \\
\hline \multirow[t]{9}{*}{1,9} & \multirow[t]{3}{*}{5} & 2 & 85,63 & 0,91 & 81,42 & 0,81 & 81,42 & 0,81 \\
\hline & & 20 & 61,71 & 0,49 & 81,89 & 0,82 & 81,89 & 0,82 \\
\hline & & 100 & 50,71 & 0,37 & 81,24 & 0,81 & 81,24 & 0,81 \\
\hline & \multirow[t]{3}{*}{20} & 2 & 99,54 & 0,97 & 91,12 & 0,54 & 91,18 & 0,55 \\
\hline & & 20 & 88,10 & 0,43 & 92,74 & 0,52 & 92,76 & 0,52 \\
\hline & & 100 & 66,44 & 0,26 & 92,86 & 0,51 & 92,86 & 0,51 \\
\hline & \multirow[t]{3}{*}{100} & 2 & 100,00 & 0,93 & 86,38 & 0,30 & 86,38 & 0,30 \\
\hline & & 20 & 99,12 & 0,35 & 94,76 & 0,25 & 94,76 & 0,25 \\
\hline & & 100 & 89,40 & 0,20 & 95,00 & 0,24 & 95,00 & 0,24 \\
\hline
\end{tabular}


Tabela 3.10: Porcentagem de cobertura (\%) e amplitude (A) dos intervalos de $95 \%$ de confiança para o parâmetro $X_{0}$, quando $\sigma_{\delta}^{2}=0,1$ e desconhecida e $\sigma_{\epsilon}^{2}=0,4$.

\begin{tabular}{|c|c|c|c|c|c|c|c|c|}
\hline \multirow[t]{2}{*}{$X_{0}$} & \multirow[t]{2}{*}{$n$} & \multirow[t]{2}{*}{$k$} & \multicolumn{2}{|c|}{ M-usual } & \multicolumn{4}{|c|}{ M-proposto } \\
\hline & & & $\%$ & A & $\% 1$ & $\mathrm{~A}_{1}$ & $\% 2$ & $\mathrm{~A}_{2}$ \\
\hline \multirow[t]{9}{*}{0,01} & \multirow[t]{3}{*}{5} & 2 & 84,01 & 2,02 & 80,65 & 2,03 & 80,87 & 2,05 \\
\hline & & 20 & 86,99 & 1,42 & 91,15 & 1,83 & 91,26 & 1,83 \\
\hline & & 100 & 87,17 & 1,57 & 91,99 & 2,14 & 92,03 & 2,14 \\
\hline & \multirow[t]{3}{*}{20} & 2 & 97,25 & 1,38 & 84,32 & 0,93 & 84,67 & 0,94 \\
\hline & & 20 & 91,64 & 0,76 & 93,61 & 0,83 & 93,69 & 0,83 \\
\hline & & 100 & 87,55 & 0,62 & 93,13 & 0,79 & 93,17 & 0,79 \\
\hline & \multirow[t]{3}{*}{100} & 2 & 99,07 & 1,27 & 74,24 & 0,60 & 74,39 & 0,61 \\
\hline & & 20 & 96,92 & 0,50 & 94,48 & 0,44 & 94,50 & 0,44 \\
\hline & & 100 & 92,08 & 0,34 & 94,18 & 0,37 & 94,18 & 0,37 \\
\hline \multirow[t]{9}{*}{0,8} & \multirow[t]{3}{*}{5} & 2 & 87,07 & 1,25 & 79,12 & 1,01 & 79,61 & 1,05 \\
\hline & & 20 & 89,71 & 1,06 & 94,27 & 1,33 & 94,50 & 1,40 \\
\hline & & 100 & 88,60 & 0,73 & 94,73 & 0,98 & 94,77 & 0,99 \\
\hline & \multirow[t]{3}{*}{20} & 2 & 97,86 & 1,23 & 77,51 & 0,65 & 78,09 & 0,67 \\
\hline & & 20 & 93,85 & 0,49 & 94,40 & 0,50 & 94,73 & 0,51 \\
\hline & & 100 & 88,80 & 0,35 & 94,56 & 0,44 & 94,73 & 0,44 \\
\hline & \multirow[t]{3}{*}{100} & 2 & 99,29 & 1,24 & 64,46 & 0,51 & 64,61 & 0,51 \\
\hline & & 20 & 98,36 & 0,41 & 94,33 & 0,33 & 94,51 & 0,33 \\
\hline & & 100 & 94,52 & 0,22 & 94,74 & 0,22 & 94,76 & 0,22 \\
\hline \multirow[t]{9}{*}{1,9} & \multirow[t]{3}{*}{5} & 2 & 85,18 & 1,57 & 81,65 & 1,48 & 81,94 & 1,50 \\
\hline & & 20 & 86,52 & 1,45 & 91,12 & 1,91 & 91,15 & 1,92 \\
\hline & & 100 & 87,43 & 4,87 & 92,94 & 7,55 & 92,98 & 7,56 \\
\hline & \multirow[t]{3}{*}{20} & 2 & 97,12 & 1,36 & 83,94 & 0,89 & 84,22 & 0,90 \\
\hline & & 20 & 91,77 & 0,72 & 93,33 & 0,78 & 93,48 & 0,79 \\
\hline & & 100 & 87,67 & 0,58 & 93,50 & 0,74 & 93,54 & 0,74 \\
\hline & \multirow[t]{3}{*}{100} & 2 & 99,09 & 1,27 & 73,10 & 0,59 & 73,25 & 0,59 \\
\hline & & 20 & 97,43 & 0,49 & 94,74 & 0,42 & 94,82 & 0,42 \\
\hline & & 100 & 92,18 & 0,32 & 94,48 & 0,35 & 94,56 & 0,35 \\
\hline
\end{tabular}


Tabela 3.11: Vício e erro quadrático médio empírico de $\hat{\alpha}$ e $\hat{\beta}$, quando $\sigma_{\delta}^{2}=0,01$ e conhecida e $\sigma_{\epsilon}^{2}=0,04$.

\begin{tabular}{|c|c|c|c|c|c|c|c|c|c|c|}
\hline \multirow{3}{*}{$X_{0}$} & \multirow{3}{*}{$n$} & \multirow{3}{*}{$k$} & \multicolumn{4}{|c|}{$\hat{\alpha}$} & \multicolumn{4}{|c|}{$\hat{\boldsymbol{\beta}}$} \\
\hline & & & \multicolumn{2}{|c|}{ M-usual } & \multicolumn{2}{|c|}{ M-proposto } & \multicolumn{2}{|c|}{ M-usual } & \multicolumn{2}{|c|}{ M-proposto } \\
\hline & & & Vício & EQM & Vício & EQM & Vício & EQM & Vício & EQM \\
\hline \multirow[t]{9}{*}{0,01} & \multirow[t]{3}{*}{5} & 2 & 0,0300 & 0,0480 & $-0,0330$ & 0,0310 & 0,0280 & 0,0470 & $-0,0320$ & 0,0300 \\
\hline & & 20 & 0,0330 & 0,0490 & $-0,0300$ & 0,0320 & 0,0380 & 0,0490 & $-0,0350$ & 0,0320 \\
\hline & & 100 & 0,0310 & 0,0480 & $-0,0310$ & 0,0300 & 0,0360 & 0,0480 & $-0,0360$ & 0,0300 \\
\hline & \multirow[t]{3}{*}{20} & 2 & 0,0086 & 0,0150 & $-0,0077$ & 0,0110 & 0,0055 & 0,0150 & $-0,0046$ & 0,0110 \\
\hline & & 20 & 0,0053 & 0,0150 & $-0,0052$ & 0,0110 & 0,0072 & 0,0150 & $-0,0070$ & 0,0110 \\
\hline & & 100 & 0,0052 & 0,0140 & $-0,0049$ & 0,0110 & 0,0093 & 0,0140 & $-0,0090$ & 0,0110 \\
\hline & \multirow[t]{3}{*}{100} & 2 & 0,0006 & 0,0030 & $-0,0003$ & 0,0023 & $-0,0006$ & 0,0031 & 0,0009 & 0,0023 \\
\hline & & 20 & $-0,0001$ & 0,0031 & $-0,0002$ & 0,0023 & $-0,0003$ & 0,0030 & 0,0000 & 0,0023 \\
\hline & & 100 & 0,0005 & 0,0031 & $-0,0003$ & 0,0023 & 0,0011 & 0,0030 & $-0,0009$ & 0,0023 \\
\hline \multirow[t]{9}{*}{0,8} & \multirow[t]{3}{*}{5} & 2 & 0,0390 & 0,0490 & $-0,0390$ & 0,0330 & 0,0380 & 0,0480 & $-0,0380$ & 0,0320 \\
\hline & & 20 & 0,0240 & 0,0490 & $-0,0290$ & 0,0310 & 0,0290 & 0,0490 & $-0,0340$ & 0,0320 \\
\hline & & 100 & 0,0380 & 0,0480 & $-0,0370$ & 0,0320 & 0,0430 & 0,0490 & $-0,0420$ & 0,0320 \\
\hline & \multirow[t]{3}{*}{20} & 2 & 0,0091 & 0,0150 & $-0,0082$ & 0,0110 & 0,0059 & 0,0150 & $-0,0050$ & 0,0110 \\
\hline & & 20 & 0,0039 & 0,0150 & $-0,0038$ & 0,0110 & 0,0058 & 0,0140 & $-0,0057$ & 0,0110 \\
\hline & & 100 & 0,0042 & 0,0140 & $-0,0043$ & 0,0110 & 0,0083 & 0,0140 & $-0,0084$ & 0,0100 \\
\hline & \multirow[t]{3}{*}{100} & 2 & 0,0002 & 0,0032 & $-0,0005$ & 0,0024 & $-0,0010$ & 0,0032 & 0,0007 & 0,0024 \\
\hline & & 20 & 0,0004 & 0,0031 & $-0,0004$ & 0,0024 & 0,0003 & 0,0031 & $-0,0003$ & 0,0023 \\
\hline & & 100 & 0,0004 & 0,0032 & 0,0000 & 0,0024 & 0,0012 & 0,0031 & $-0,0007$ & 0,0023 \\
\hline \multirow[t]{9}{*}{1,9} & \multirow[t]{3}{*}{5} & 2 & 0,0310 & 0,0490 & $-0,0350$ & 0,0340 & 0,0310 & 0,0480 & $-0,0340$ & 0,0330 \\
\hline & & 20 & 0,0300 & 0,0470 & $-0,0310$ & 0,0330 & 0,0350 & 0,0470 & $-0,0360$ & 0,0330 \\
\hline & & 100 & 0,0390 & 0,0480 & $-0,0320$ & 0,0320 & 0,0450 & 0,0490 & $-0,0380$ & 0,0330 \\
\hline & \multirow[t]{3}{*}{20} & 2 & 0,0100 & 0,0150 & $-0,0093$ & 0,0110 & 0,0070 & 0,0150 & $-0,0059$ & 0,0110 \\
\hline & & 20 & 0,0032 & 0,0150 & $-0,0039$ & 0,0110 & 0,0047 & 0,0150 & $-0,0054$ & 0,0110 \\
\hline & & 100 & 0,0027 & 0,0140 & $-0,0035$ & 0,0100 & 0,0068 & 0,0140 & $-0,0076$ & 0,0099 \\
\hline & \multirow[t]{3}{*}{100} & 2 & 0,0012 & 0,0031 & $-0,0012$ & 0,0023 & 0,0000 & 0,0031 & 0,0000 & 0,0023 \\
\hline & & 20 & $-0,0005$ & 0,0032 & 0,0001 & 0,0024 & $-0,0006$ & 0,0032 & 0,0003 & 0,0023 \\
\hline & & 100 & 0,0002 & 0,0031 & 0,0004 & 0,0023 & 0,0007 & 0,0031 & $-0,0001$ & 0,0023 \\
\hline
\end{tabular}


Tabela 3.12: Vício e erro quadrático médio empírico de $\hat{\alpha}$ e $\hat{\beta}$, quando $\sigma_{\delta}^{2}=0,01$ e conhecida e $\sigma_{\epsilon}^{2}=0,4$.

\begin{tabular}{|c|c|c|c|c|c|c|c|c|c|c|}
\hline \multirow{3}{*}{$X_{0}$} & \multirow{3}{*}{$n$} & \multirow{3}{*}{$k$} & \multicolumn{4}{|c|}{$\hat{\alpha}$} & \multicolumn{4}{|c|}{$\hat{\beta}$} \\
\hline & & & \multicolumn{2}{|c|}{ M-usual } & \multicolumn{2}{|c|}{ M-proposto } & \multicolumn{2}{|c|}{ M-usual } & \multicolumn{2}{|c|}{ M-proposto } \\
\hline & & & Vício & EQM & Vício & EQM & Vício & EQM & Vício & EQM \\
\hline \multirow[t]{9}{*}{0,01} & \multirow[t]{3}{*}{5} & 2 & 0,0290 & 0,2700 & $-0,0220$ & 0,1760 & 0,0260 & 0,2700 & $-0,0190$ & 0,1770 \\
\hline & & 20 & 0,0230 & 0,2620 & $-0,0230$ & 0,1750 & 0,0350 & 0,2610 & $-0,0350$ & 0,1740 \\
\hline & & 100 & 0,0210 & 0,2540 & $-0,0150$ & 0,1640 & 0,0350 & 0,2530 & $-0,0300$ & 0,1630 \\
\hline & \multirow[t]{3}{*}{20} & 2 & 0,0017 & 0,0820 & $-0,0002$ & 0,0590 & $-0,0008$ & 0,0820 & 0,0023 & 0,0590 \\
\hline & & 20 & 0,0016 & 0,0820 & 0,0004 & 0,0590 & 0,0032 & 0,0810 & $-0,0012$ & 0,0590 \\
\hline & & 100 & 0,0054 & 0,0840 & $-0,0041$ & 0,0600 & 0,0097 & 0,0830 & $-0,0085$ & 0,0590 \\
\hline & \multirow[t]{3}{*}{100} & 2 & $-0,0016$ & 0,0170 & 0,0015 & 0,0130 & $-0,0022$ & 0,0170 & 0,0022 & 0,0130 \\
\hline & & 20 & 0,0008 & 0,0170 & 0,0002 & 0,0130 & 0,0005 & 0,0170 & 0,0005 & 0,0130 \\
\hline & & 100 & 0,0011 & 0,0170 & $-0,0009$ & 0,0130 & 0,0016 & 0,0170 & $-0,0013$ & 0,0130 \\
\hline \multirow[t]{9}{*}{0,8} & \multirow[t]{3}{*}{5} & 2 & 0,0320 & 0,2590 & $-0,0260$ & 0,1750 & 0,0300 & 0,2590 & $-0,0240$ & 0,1750 \\
\hline & & 20 & 0,0190 & 0,2630 & $-0,0240$ & 0,1660 & 0,0320 & 0,2610 & $-0,0360$ & 0,1650 \\
\hline & & 100 & 0,0170 & 0,2690 & $-0,0240$ & 0,1790 & 0,0320 & 0,2670 & $-0,0390$ & 0,1780 \\
\hline & \multirow[t]{3}{*}{20} & 2 & $-0,0050$ & 0,0820 & 0,0029 & 0,0600 & $-0,0075$ & 0,0820 & 0,0054 & 0,0610 \\
\hline & & 20 & $-0,0063$ & 0,0780 & 0,0048 & 0,0580 & $-0,0047$ & 0,0780 & 0,0031 & 0,0580 \\
\hline & & 100 & $-0,0097$ & 0,0820 & 0,0053 & 0,0600 & $-0,0051$ & 0,0810 & 0,0008 & 0,0600 \\
\hline & \multirow[t]{3}{*}{100} & 2 & $-0,0011$ & 0,0170 & 0,0013 & 0,0130 & $-0,0017$ & 0,0170 & 0,0019 & 0,0130 \\
\hline & & 20 & $-0,0018$ & 0,0170 & 0,0013 & 0,0130 & $-0,0021$ & 0,0170 & 0,0015 & 0,0130 \\
\hline & & 100 & $-0,0008$ & 0,0170 & 0,0006 & 0,0130 & $-0,0004$ & 0,0170 & 0,0002 & 0,0130 \\
\hline \multirow[t]{9}{*}{1,9} & \multirow[t]{3}{*}{5} & 2 & 0,0400 & 0,2680 & $-0,0350$ & 0,1790 & 0,0370 & 0,2690 & $-0,0320$ & 0,1800 \\
\hline & & 20 & 0,0290 & 0,2600 & $-0,0260$ & 0,1680 & 0,0410 & 0,2590 & $-0,0390$ & 0,1670 \\
\hline & & 100 & 0,0180 & 0,2630 & $-0,0180$ & 0,1740 & 0,0330 & 0,2620 & $-0,0330$ & 0,1730 \\
\hline & \multirow[t]{3}{*}{20} & 2 & $-0,0003$ & 0,0820 & 0,0012 & 0,0610 & $-0,0030$ & 0,0830 & 0,0039 & 0,0610 \\
\hline & & 20 & $-0,0050$ & 0,0790 & 0,0020 & 0,0590 & $-0,0030$ & 0,0790 & 0,0000 & 0,0590 \\
\hline & & 100 & 0,0022 & 0,0850 & 0,0007 & 0,0610 & 0,0066 & 0,0840 & $-0,0037$ & 0,0610 \\
\hline & \multirow[t]{3}{*}{100} & 2 & 0,0018 & 0,0170 & $-0,0011$ & 0,0130 & 0,0011 & 0,0170 & $-0,0005$ & 0,0130 \\
\hline & & 20 & 0,0004 & 0,0170 & $-0,0003$ & 0,0130 & 0,0000 & 0,0170 & 0,0001 & 0,0130 \\
\hline & & 100 & $-0,0012$ & 0,0180 & 0,0002 & 0,0130 & $-0,0008$ & 0,0180 & $-0,0002$ & 0,0130 \\
\hline
\end{tabular}


Tabela 3.13: Vício e erro quadrático médio empírico de $\hat{\alpha}$ e $\hat{\beta}$, quando $\sigma_{\delta}^{2}=0,1$ e conhecida e $\sigma_{\epsilon}^{2}=0,04$.

\begin{tabular}{|c|c|c|c|c|c|c|c|c|c|c|}
\hline \multirow{3}{*}{$X_{0}$} & \multirow{3}{*}{$n$} & \multirow{3}{*}{$k$} & \multicolumn{4}{|c|}{$\hat{\alpha}$} & \multicolumn{4}{|c|}{$\hat{\boldsymbol{\beta}}$} \\
\hline & & & \multicolumn{2}{|c|}{ M-usual } & \multicolumn{2}{|c|}{ M-proposto } & \multicolumn{2}{|c|}{ M-usual } & \multicolumn{2}{|c|}{ M-proposto } \\
\hline & & & Vício & EQM & Vício & EQM & Vício & EQM & Vício & EQM \\
\hline \multirow[t]{9}{*}{0,01} & \multirow[t]{3}{*}{5} & 2 & 0,4540 & 0,4720 & $-0,4340$ & 0,3880 & 0,4120 & 0,4150 & $-0,3910$ & 0,3300 \\
\hline & & 20 & 0,1490 & 0,2980 & $-0,1410$ & 0,2130 & 0,1647 & 0,6977 & $-1,6400$ & 0,6845 \\
\hline & & 100 & 0,2200 & 0,2710 & $-0,2170$ & 0,1820 & 0,3560 & 0,6330 & $-0,3530$ & 0,5230 \\
\hline & \multirow[t]{3}{*}{20} & 2 & 0,1470 & 0,0960 & $-0,1530$ & 0,0820 & 0,1250 & 0,0790 & $-0,1310$ & 0,0650 \\
\hline & & 20 & 0,0580 & 0,0710 & $-0,0650$ & 0,0440 & 0,0610 & 0,0700 & $-0,0680$ & 0,0440 \\
\hline & & 100 & 0,0610 & 0,0590 & $-0,0620$ & 0,0420 & 0,1060 & 0,1990 & $-0,1070$ & 0,1860 \\
\hline & \multirow[t]{3}{*}{100} & 2 & 0,0340 & 0,0180 & $-0,0350$ & 0,0140 & 0,0280 & 0,0150 & $-0,0290$ & 0,0110 \\
\hline & & 20 & 0,0180 & 0,0140 & $-0,0190$ & 0,0100 & 0,0180 & 0,0140 & $-0,0200$ & 0,0092 \\
\hline & & 100 & 0,0097 & 0,0130 & $-0,0069$ & 0,0085 & 0,0140 & 0,0130 & $-0,0110$ & 0,0083 \\
\hline \multirow[t]{9}{*}{0,8} & \multirow[t]{3}{*}{5} & 2 & 0,3770 & 0,4090 & $-0,3530$ & 0,3130 & 0,3400 & 0,3610 & $-0,3160$ & 0,2670 \\
\hline & & 20 & 0,1250 & 0,2980 & $-0,1110$ & 0,2110 & 1,7490 & 7,4140 & $-1,7350$ & 7,4020 \\
\hline & & 100 & 0,2220 & 0,3100 & $-0,2250$ & 0,2040 & 0,3870 & 0,6930 & $-0,3900$ & 0,6330 \\
\hline & \multirow[t]{3}{*}{20} & 2 & 0,1580 & 0,1050 & $-0,1530$ & 0,0800 & 0,1320 & 0,0880 & $-0,1270$ & 0,0630 \\
\hline & & 20 & 0,0800 & 0,0690 & $-0,0740$ & 0,0480 & 0,0830 & 0,0700 & $-0,0760$ & 0,0480 \\
\hline & & 100 & 0,0650 & 0,0610 & $-0,0660$ & 0,0460 & 0,0950 & 0,1620 & $-0,0960$ & 0,1450 \\
\hline & \multirow[t]{3}{*}{100} & 2 & 0,0320 & 0,0180 & $-0,0330$ & 0,0130 & 0,0260 & 0,0160 & $-0,0260$ & 0,0100 \\
\hline & & 20 & 0,0170 & 0,0130 & $-0,0170$ & 0,0093 & 0,0180 & 0,0130 & $-0,0180$ & 0,0087 \\
\hline & & 100 & 0,0140 & 0,0120 & $-0,0140$ & 0,0085 & 0,0180 & 0,0120 & $-0,0190$ & 0,0083 \\
\hline \multirow[t]{9}{*}{1,9} & \multirow[t]{3}{*}{5} & 2 & 0,4060 & 0,4180 & $-0,4130$ & 0,3510 & 0,3770 & 0,3700 & $-0,3830$ & 0,3030 \\
\hline & & 20 & 0,1340 & 0,2930 & $-0,1400$ & 0,2120 & 1,5940 & 6,7280 & $-1,6000$ & 6,6810 \\
\hline & & 100 & 0,2100 & 0,2650 & $-0,2190$ & 0,1840 & 0,3680 & 0,6810 & $-0,3760$ & 0,5760 \\
\hline & \multirow[t]{3}{*}{20} & 2 & 0,1450 & 0,1050 & $-0,1510$ & 0,0840 & 0,1180 & 0,0870 & $-0,1240$ & 0,0650 \\
\hline & & 20 & 0,1340 & 0,2930 & $-0,1400$ & 0,2120 & 1,5940 & 6,7280 & $-1,6000$ & 6,6810 \\
\hline & & 100 & 0,2100 & 0,2650 & $-0,2190$ & 0,1840 & 0,3680 & 0,6810 & $-0,3760$ & 0,5760 \\
\hline & \multirow[t]{3}{*}{100} & 2 & 0,0370 & 0,0190 & $-0,0380$ & 0,0140 & 0,0290 & 0,0160 & $-0,0300$ & 0,0110 \\
\hline & & 20 & 0,0160 & 0,0140 & $-0,0180$ & 0,0097 & 0,0170 & 0,0130 & $-0,0200$ & 0,0089 \\
\hline & & 100 & 0,0130 & 0,0150 & $-0,0120$ & 0,0098 & 0,0170 & 0,0140 & $-0,0160$ & 0,0095 \\
\hline
\end{tabular}


Tabela 3.14: Vício e erro quadrático médio empírico de $\hat{\alpha}$ e $\hat{\beta}$, quando $\sigma_{\delta}^{2}=0,1$ e conhecida e $\sigma_{\epsilon}^{2}=0,4$.

\begin{tabular}{|c|c|c|c|c|c|c|c|c|c|c|}
\hline \multirow{3}{*}{$X_{0}$} & \multirow{3}{*}{$n$} & \multirow{3}{*}{$k$} & \multicolumn{4}{|c|}{$\hat{\alpha}$} & \multicolumn{4}{|c|}{$\hat{\boldsymbol{\beta}}$} \\
\hline & & & \multicolumn{2}{|c|}{ M-usual } & \multicolumn{2}{|c|}{ M-proposto } & \multicolumn{2}{|c|}{ M-usual } & \multicolumn{2}{|c|}{ M-proposto } \\
\hline & & & Vício & EQM & Vício & EQM & Vício & EQM & Vício & EQM \\
\hline \multirow[t]{9}{*}{0,01} & \multirow[t]{3}{*}{5} & 2 & 0,3440 & 0,5610 & $-0,3490$ & 0,4020 & 0,3260 & 0,5390 & $-0,3310$ & 0,3800 \\
\hline & & 20 & 0,2760 & 0,5160 & $-0,2660$ & 0,3430 & 0,3720 & 0,8410 & $-0,3610$ & 0,6560 \\
\hline & & 100 & 0,2520 & 0,5020 & $-0,2590$ & 0,3460 & 0,3130 & 0,5890 & $-0,3200$ & 0,4420 \\
\hline & \multirow[t]{3}{*}{20} & 2 & 0,0830 & 0,1450 & $-0,0880$ & 0,1050 & 0,0560 & 0,1400 & $-0,0610$ & 0,0990 \\
\hline & & 20 & 0,0490 & 0,1390 & $-0,0510$ & 0,0980 & 0,0630 & 0,1290 & $-0,0650$ & 0,0870 \\
\hline & & 100 & 0,0360 & 0,1350 & $-0,0380$ & 0,0960 & 0,0850 & 0,2220 & $-0,0870$ & 0,1830 \\
\hline & \multirow[t]{3}{*}{100} & 2 & 0,0068 & 0,0310 & $-0,0057$ & 0,0230 & $-0,0050$ & 0,0320 & 0,0062 & 0,0240 \\
\hline & & 20 & $-0,0003$ & 0,0310 & 0,0002 & 0,0230 & $-0,0014$ & 0,0290 & 0,0013 & 0,0210 \\
\hline & & 100 & 0,0000 & 0,0320 & 0,0010 & 0,0240 & 0,0068 & 0,0270 & $-0,0057$ & 0,0190 \\
\hline \multirow[t]{9}{*}{0,8} & \multirow[t]{3}{*}{5} & 2 & 0,3460 & 0,5700 & $-0,3550$ & 0,4080 & 0,3200 & 0,5400 & $-0,3290$ & 0,3790 \\
\hline & & 20 & 0,2460 & 0,4930 & $-0,2500$ & 0,3560 & 0,3700 & 1,0080 & $-0,3730$ & 0,8660 \\
\hline & & 100 & 0,2570 & 0,5140 & $-0,2390$ & 0,3280 & 0,3350 & 0,6750 & $-0,3170$ & 0,4880 \\
\hline & \multirow[t]{3}{*}{20} & 2 & 0,0690 & 0,1450 & $-0,0690$ & 0,1060 & 0,0430 & 0,1400 & $-0,0420$ & 0,1010 \\
\hline & & 20 & 0,0590 & 0,1360 & $-0,0530$ & 0,0990 & 0,0710 & 0,1260 & $-0,0650$ & 0,0890 \\
\hline & & 100 & 0,0370 & 0,1370 & $-0,0380$ & 0,0970 & 0,0870 & 0,2340 & $-0,0890$ & 0,1920 \\
\hline & \multirow[t]{3}{*}{100} & 2 & 0,0003 & 0,0300 & $-0,0008$ & 0,0220 & $-0,0120$ & 0,0310 & 0,0120 & 0,0240 \\
\hline & & 20 & 0,0003 & 0,0310 & 0,0013 & 0,0230 & 0,0000 & 0,0280 & 0,0016 & 0,0200 \\
\hline & & 100 & $-0,0006$ & 0,0320 & 0,0001 & 0,0240 & 0,0048 & 0,0270 & $-0,0053$ & 0,0190 \\
\hline \multirow[t]{9}{*}{1,9} & \multirow[t]{3}{*}{5} & 2 & 0,3190 & 0,5130 & $-0,3320$ & 0,3710 & 0,2980 & 0,4950 & $-0,3120$ & 0,3530 \\
\hline & & 20 & 0,2710 & 0,5160 & $-0,2580$ & 0,3450 & 0,3900 & 0,9970 & $-0,3780$ & 0,8160 \\
\hline & & 100 & 0,0130 & 0,0150 & $-0,0120$ & 0,0098 & 0,0170 & 0,0140 & $-0,0160$ & 0,0095 \\
\hline & \multirow[t]{3}{*}{20} & 2 & 0,0840 & 0,1470 & $-0,0820$ & 0,1050 & 0,0560 & 0,1400 & $-0,0530$ & 0,0980 \\
\hline & & 20 & 0,2710 & 0,5160 & $-0,2580$ & 0,3450 & 0,3900 & 0,9970 & $-0,3780$ & 0,8160 \\
\hline & & 100 & 0,0350 & 0,1380 & $-0,0350$ & 0,0950 & 0,0850 & 0,2200 & $-0,0850$ & 0,1750 \\
\hline & \multirow[t]{3}{*}{100} & 2 & $-0,0001$ & 0,0320 & $-0,0004$ & 0,0240 & $-0,0120$ & 0,0330 & 0,0120 & 0,0250 \\
\hline & & 20 & $-0,0053$ & 0,0320 & 0,0057 & 0,0240 & $-0,0047$ & 0,0290 & 0,0051 & 0,0210 \\
\hline & & 100 & $-0,0021$ & 0,0310 & 0,0000 & 0,0230 & 0,0044 & 0,0270 & $-0,0066$ & 0,0190 \\
\hline
\end{tabular}


Tabela 3.15: Vício e erro quadrático médio empírico, média das variâncias estimadas e variância teórica do estimador $\hat{X}_{0}$, para $\sigma_{\delta}^{2}=0,01$ e conhecida e $\sigma_{\epsilon}^{2}=0,04$.

\begin{tabular}{|c|c|c|c|c|c|c|c|c|c|}
\hline \multirow{3}{*}{$X_{0}$} & \multirow{3}{*}{$n$} & \multirow{3}{*}{$k$} & \multicolumn{4}{|c|}{ Empírico } & \multirow{3}{*}{$\begin{array}{c}\text { Teórico } \\
\text { M-proposto } \\
V\left(\hat{X}_{0}\right)\end{array}$} & \multicolumn{2}{|c|}{ Média de $\hat{V}\left(X_{0}\right)$} \\
\hline & & & \multicolumn{2}{|c|}{ M-usual } & \multicolumn{2}{|c|}{ M-proposto } & & \multirow[t]{2}{*}{ M-usual } & \multirow[t]{2}{*}{ M-proposto } \\
\hline & & & Vício & EQM & Vício & EQM & & & \\
\hline \multirow[t]{9}{*}{0,01} & \multirow[t]{3}{*}{5} & 2 & $-0,0290$ & 0,0210 & $-0,0280$ & 0,0210 & 0,0170 & 0,0180 & 0,0160 \\
\hline & & 20 & $-0,0290$ & 0,0140 & $-0,0320$ & 0,0140 & 0,0120 & 0,0081 & 0,0130 \\
\hline & & 100 & $-0,0240$ & 0,0140 & $-0,0270$ & 0,0140 & 0,0120 & 0,0070 & 0,0130 \\
\hline & \multirow[t]{3}{*}{20} & 2 & $-0,0081$ & 0,0091 & $-0,0064$ & 0,0090 & 0,0086 & 0,0130 & 0,0076 \\
\hline & & 20 & $-0,0060$ & 0,0043 & $-0,0072$ & 0,0043 & 0,0041 & 0,0034 & 0,0041 \\
\hline & & 100 & $-0,0038$ & 0,0038 & $-0,0060$ & 0,0038 & 0,0037 & 0,0022 & 0,0038 \\
\hline & \multirow[t]{3}{*}{100} & 2 & $-0,0011$ & 0,0056 & $-0,0005$ & 0,0056 & 0,0058 & 0,0100 & 0,0053 \\
\hline & & 20 & $-0,0002$ & 0,0013 & $-0,0001$ & 0,0013 & 0,0013 & 0,0016 & 0,0012 \\
\hline & & 100 & $-0,0009$ & 0,0009 & $-0,0012$ & 0,0009 & 0,0009 & 0,0007 & 0,0009 \\
\hline \multirow[t]{9}{*}{0,8} & \multirow[t]{3}{*}{5} & 2 & $-0,0074$ & 0,0110 & $-0,0072$ & 0,0100 & 0,0093 & 0,0120 & 0,0085 \\
\hline & & 20 & $-0,0046$ & 0,0051 & $-0,0051$ & 0,0052 & 0,0048 & 0,0032 & 0,0049 \\
\hline & & 100 & $-0,0076$ & 0,0048 & $-0,0082$ & 0,0048 & 0,0044 & 0,0025 & 0,0046 \\
\hline & \multirow[t]{3}{*}{20} & 2 & $-0,0034$ & 0,0063 & $-0,0031$ & 0,0063 & 0,0061 & 0,0100 & 0,0052 \\
\hline & & 20 & 0,0001 & 0,0016 & 0,0000 & 0,0016 & 0,0016 & 0,0015 & 0,0016 \\
\hline & & 100 & $-0,0009$ & 0,0012 & $-0,0013$ & 0,0012 & 0,0012 & 0,0008 & 0,0012 \\
\hline & \multirow[t]{3}{*}{100} & 2 & 0,0000 & 0,0053 & 0,0002 & 0,0053 & 0,0052 & 0,0099 & 0,0048 \\
\hline & & 20 & 0,0000 & 0,0007 & 0,0000 & 0,0007 & 0,0007 & 0,0011 & 0,0007 \\
\hline & & 100 & $-0,0001$ & 0,0003 & $-0,0002$ & 0,0003 & 0,0003 & 0,0003 & 0,0003 \\
\hline \multirow[t]{9}{*}{1,9} & \multirow[t]{3}{*}{5} & 2 & 0,0200 & 0,0180 & 0,0200 & 0,0180 & 0,0150 & 0,0170 & 0,0140 \\
\hline & & 20 & 0,0240 & 0,0120 & 0,0260 & 0,0130 & 0,0110 & 0,0071 & 0,0120 \\
\hline & & 100 & 0,0200 & 0,0130 & 0,0230 & 0,0130 & 0,0100 & 0,0062 & 0,0110 \\
\hline & \multirow[t]{3}{*}{20} & 2 & 0,0037 & 0,0082 & 0,0021 & 0,0081 & 0,0082 & 0,0120 & 0,0071 \\
\hline & & 20 & 0,0059 & 0,0037 & 0,0066 & 0,0037 & 0,0037 & 0,0030 & 0,0036 \\
\hline & & 100 & 0,0033 & 0,0032 & 0,0051 & 0,0032 & 0,0032 & 0,0020 & 0,0033 \\
\hline & \multirow[t]{3}{*}{100} & 2 & 0,0020 & 0,0058 & 0,0015 & 0,0057 & 0,0057 & 0,0100 & 0,0053 \\
\hline & & 20 & 0,0003 & 0,0012 & 0,0002 & 0,0012 & 0,0012 & 0,0015 & 0,0011 \\
\hline & & 100 & 0,0003 & 0,0008 & 0,0006 & 0,0008 & 0,0008 & 0,0006 & 0,0008 \\
\hline
\end{tabular}


Tabela 3.16: Vício e erro quadrático médio empírico, média das variâncias estimadas e variância teórica do estimador $\hat{X}_{0}$, para $\sigma_{\delta}^{2}=0,01$ e conhecida e $\sigma_{\epsilon}^{2}=0,4$.

\begin{tabular}{|c|c|c|c|c|c|c|c|c|c|}
\hline \multirow{3}{*}{$X_{0}$} & \multirow{3}{*}{$n$} & \multirow{3}{*}{$k$} & \multicolumn{4}{|c|}{ Empírico } & \multirow{3}{*}{$\begin{array}{c}\text { Teórico } \\
\text { M-proposto } \\
V\left(\hat{X}_{0}\right) \\
\end{array}$} & \multicolumn{2}{|c|}{ Média de $\hat{V}\left(X_{0}\right)$} \\
\hline & & & \multicolumn{2}{|c|}{ M-usual } & \multicolumn{2}{|c|}{ M-proposto } & & \multirow[t]{2}{*}{ M-usual } & \multirow[t]{2}{*}{ M-proposto } \\
\hline & & & Vício & EQM & Vício & EQM & & & \\
\hline \multirow[t]{9}{*}{0,01} & \multirow[t]{3}{*}{5} & 2 & $-0,0700$ & 0,1740 & $-0,0680$ & 0,1740 & 0,1150 & 0,1220 & 0,1190 \\
\hline & & 20 & $-0,0640$ & 0,1150 & $-0,0700$ & 0,1170 & 0,0700 & 0,1640 & 0,1740 \\
\hline & & 100 & $-0,0560$ & 0,0970 & $-0,0640$ & 0,0990 & 0,0660 & 0,1050 & 0,1130 \\
\hline & \multirow[t]{3}{*}{20} & 2 & $-0,0150$ & 0,0760 & $-0,0140$ & 0,0760 & 0,0700 & 0,0700 & 0,0650 \\
\hline & & 20 & $-0,0190$ & 0,0280 & $-0,0200$ & 0,0280 & 0,0250 & 0,0260 & 0,0270 \\
\hline & & 100 & $-0,0150$ & 0,0240 & $-0,0170$ & 0,0240 & 0,0210 & 0,0220 & 0,0240 \\
\hline & \multirow[t]{3}{*}{100} & 2 & $-0,0056$ & 0,0540 & $-0,0053$ & 0,0540 & 0,0540 & 0,0580 & 0,0530 \\
\hline & & 20 & $-0,0045$ & 0,0098 & $-0,0043$ & 0,0098 & 0,0093 & 0,0096 & 0,0092 \\
\hline & & 100 & $-0,0027$ & 0,0057 & $-0,0029$ & 0,0057 & 0,0053 & 0,0052 & 0,0053 \\
\hline \multirow[t]{9}{*}{0,8} & \multirow[t]{3}{*}{5} & 2 & $-0,0120$ & 0,0920 & $-0,0120$ & 0,0920 & 0,0740 & 0,0650 & 0,0610 \\
\hline & & 20 & $-0,0091$ & 0,0360 & $-0,0100$ & 0,0370 & 0,0290 & 0,0320 & 0,0340 \\
\hline & & 100 & $-0,0090$ & 0,0290 & $-0,0110$ & 0,0290 & 0,0250 & 0,0290 & 0,0320 \\
\hline & \multirow[t]{3}{*}{20} & 2 & $-0,0019$ & 0,0600 & $-0,0017$ & 0,0600 & 0,0560 & 0,0570 & 0,0510 \\
\hline & & 20 & 0,0006 & 0,0120 & 0,0005 & 0,0120 & 0,0110 & 0,0110 & 0,0110 \\
\hline & & 100 & $-0,0027$ & 0,0073 & $-0,0031$ & 0,0074 & 0,0071 & 0,0070 & 0,0075 \\
\hline & \multirow[t]{3}{*}{100} & 2 & 0,0090 & 0,0530 & 0,0090 & 0,0530 & 0,0510 & 0,0550 & 0,0500 \\
\hline & & 20 & $-0,0006$ & 0,0062 & $-0,0006$ & 0,0062 & 0,0062 & 0,0065 & 0,0061 \\
\hline & & 100 & $-0,0008$ & 0,0023 & $-0,0008$ & 0,0023 & 0,0022 & 0,0022 & 0,0022 \\
\hline \multirow[t]{9}{*}{1,9} & \multirow[t]{3}{*}{5} & 2 & 0,0750 & 0,1740 & 0,0730 & 0,1730 & 0,1080 & 0,1150 & 0,1110 \\
\hline & & 20 & 0,0530 & 0,0870 & 0,0590 & 0,0890 & 0,0630 & 0,0840 & 0,0910 \\
\hline & & 100 & 0,0530 & 0,0850 & 0,0600 & 0,0870 & 0,0590 & 0,0870 & 0,0950 \\
\hline & \multirow[t]{3}{*}{20} & 2 & 0,0130 & 0,0730 & 0,0110 & 0,0730 & 0,0680 & 0,0680 & 0,0630 \\
\hline & & 20 & 0,0130 & 0,0240 & 0,0130 & 0,0240 & 0,0230 & 0,0230 & 0,0230 \\
\hline & & 100 & 0,0130 & 0,0200 & 0,0140 & 0,0200 & 0,0190 & 0,0190 & 0,0210 \\
\hline & \multirow[t]{3}{*}{100} & 2 & 0,0072 & 0,0550 & 0,0069 & 0,0550 & 0,0540 & 0,0580 & 0,0530 \\
\hline & & 20 & 0,0026 & 0,0086 & 0,0024 & 0,0086 & 0,0087 & 0,0090 & 0,0086 \\
\hline & & 100 & 0,0022 & 0,0048 & 0,0024 & 0,0048 & 0,0047 & 0,0046 & 0,0048 \\
\hline
\end{tabular}


Tabela 3.17: Vício e erro quadrático médio empírico, média das variâncias estimadas e variância teórica do estimador $\hat{X}_{0}$, para $\sigma_{\delta}^{2}=0,1$ e conhecida e $\sigma_{\epsilon}^{2}=0,04$.

\begin{tabular}{|c|c|c|c|c|c|c|c|c|c|}
\hline \multirow{3}{*}{$X_{0}$} & \multirow{3}{*}{$n$} & \multirow{3}{*}{$k$} & \multicolumn{4}{|c|}{ Empírico } & \multirow{3}{*}{$\begin{array}{c}\text { Teórico } \\
\text { M-proposto } \\
V\left(\hat{X}_{0}\right)\end{array}$} & \multicolumn{2}{|c|}{ Média de $\hat{V}\left(X_{0}\right)$} \\
\hline & & & \multicolumn{2}{|c|}{ M-usual } & \multicolumn{2}{|c|}{ M-proposto } & & \multirow[t]{2}{*}{ M-usual } & \multirow[t]{2}{*}{ M-proposto } \\
\hline & & & Vício & EQM & Vício & EQM & & & \\
\hline \multirow[t]{9}{*}{0,01} & \multirow[t]{3}{*}{5} & 2 & $-0,4330$ & 0,5590 & $-0,3830$ & 0,4580 & 0,0590 & 0,3650 & 0,2310 \\
\hline & & 20 & $-0,1140$ & 0,1310 & 0,4140 & 1,0880 & 0,0540 & 0,0250 & 0,0760 \\
\hline & & 100 & $-0,1890$ & 0,1540 & $-0,0290$ & 0,7730 & 0,0540 & 0,0200 & 0,1250 \\
\hline & \multirow[t]{3}{*}{20} & 2 & $-0,0930$ & 0,0430 & $-0,0770$ & 0,0360 & 0,0210 & 0,0950 & 0,0380 \\
\hline & & 20 & $-0,0490$ & 0,0210 & $-0,0510$ & 0,0210 & 0,0160 & 0,0150 & 0,0180 \\
\hline & & 100 & $-0,0430$ & 0,0190 & $-0,0250$ & 0,0640 & 0,0150 & 0,0058 & 0,0170 \\
\hline & \multirow[t]{3}{*}{100} & 2 & $-0,0200$ & 0,0110 & $-0,0160$ & 0,0097 & 0,0084 & 0,0630 & 0,0110 \\
\hline & & 20 & $-0,0077$ & 0,0041 & $-0,0085$ & 0,0039 & 0,0037 & 0,0084 & 0,0038 \\
\hline & & 100 & $-0,0066$ & 0,0037 & $-0,0087$ & 0,0037 & 0,0033 & 0,0029 & 0,0033 \\
\hline \multirow[t]{9}{*}{0,8} & \multirow[t]{3}{*}{5} & 2 & $-0,0500$ & 0,0620 & $-0,0430$ & 0,0550 & 0,0280 & 0,1150 & 0,0620 \\
\hline & & 20 & $-0,0450$ & 0,0380 & 0,0820 & 0,0810 & 0,0240 & 0,0086 & 0,0320 \\
\hline & & 100 & $-0,0530$ & 0,0510 & $-0,0069$ & 0,0980 & 0,0230 & 0,0055 & 0,0360 \\
\hline & \multirow[t]{3}{*}{20} & 2 & $-0,0280$ & 0,0150 & $-0,0240$ & 0,0140 & 0,0110 & 0,0740 & 0,0210 \\
\hline & & 20 & $-0,0079$ & 0,0068 & $-0,0085$ & 0,0069 & 0,0064 & 0,0065 & 0,0067 \\
\hline & & 100 & $-0,0055$ & 0,0065 & $-0,0030$ & 0,0079 & 0,0060 & 0,0018 & 0,0062 \\
\hline & \multirow[t]{3}{*}{100} & 2 & $-0,0030$ & 0,0067 & $-0,0021$ & 0,0065 & 0,0062 & 0,0600 & 0,0091 \\
\hline & & 20 & $-0,0018$ & 0,0018 & $-0,0018$ & 0,0017 & 0,0017 & 0,0058 & 0,0017 \\
\hline & & 100 & $-0,0021$ & 0,0013 & $-0,0025$ & 0,0013 & 0,0013 & 0,0013 & 0,0013 \\
\hline \multirow[t]{9}{*}{1,9} & \multirow[t]{3}{*}{5} & 2 & 0,3480 & 0,3770 & 0,3070 & 0,3000 & 0,0530 & 0,2850 & 0,1760 \\
\hline & & 20 & 0,1400 & 0,1630 & $-0,3310$ & 0,9370 & 0,0490 & 0,0300 & 0,0780 \\
\hline & & 100 & 0,0410 & 0,0180 & 0,0270 & 0,0510 & 0,0140 & 0,0051 & 0,0150 \\
\hline & \multirow[t]{3}{*}{20} & 2 & 0,0970 & 0,0430 & 0,0790 & 0,0350 & 0,0190 & 0,0930 & 0,0350 \\
\hline & & 20 & 0,1400 & 0,1630 & $-0,3310$ & 0,9370 & 0,0490 & 0,0300 & 0,0780 \\
\hline & & 100 & 0,1670 & 0,1300 & $-0,0005$ & 0,7090 & 0,0480 & 0,0160 & 0,1120 \\
\hline & \multirow[t]{3}{*}{100} & 2 & 0,0200 & 0,0093 & 0,0160 & 0,0087 & 0,0080 & 0,0620 & 0,0110 \\
\hline & & 20 & 0,0120 & 0,0039 & 0,0130 & 0,0037 & 0,0033 & 0,0080 & 0,0035 \\
\hline & & 100 & 0,0072 & 0,0031 & 0,0089 & 0,0031 & 0,0029 & 0,0027 & 0,0030 \\
\hline
\end{tabular}


Tabela 3.18: Vício e erro quadrático médio empírico, média das variâncias estimadas e variância teórica do estimador $\hat{X}_{0}$, para $\sigma_{\delta}^{2}=0,1$ e conhecida e $\sigma_{\epsilon}^{2}=0,4$.

\begin{tabular}{|c|c|c|c|c|c|c|c|c|c|}
\hline \multirow{3}{*}{$X_{0}$} & \multirow{3}{*}{$n$} & \multirow{3}{*}{$k$} & \multicolumn{4}{|c|}{ Empírico } & \multirow{3}{*}{$\begin{array}{c}\text { Teórico } \\
\text { M-proposto } \\
V\left(\hat{X}_{0}\right) \\
\end{array}$} & \multicolumn{2}{|c|}{ Média de $V\left(\hat{X}_{0}\right)$} \\
\hline & & & \multicolumn{2}{|c|}{ M-usual } & \multicolumn{2}{|c|}{ M-proposto } & & \multirow[t]{2}{*}{ M-usual } & \multirow[t]{2}{*}{ M-proposto } \\
\hline & & & Vício & EQM & Vício & EQM & & & \\
\hline \multirow[t]{9}{*}{0,01} & 5 & 2 & $-0,3850$ & 0,9430 & $-0,3570$ & 0,8450 & 0,1600 & 4,7870 & 3,6080 \\
\hline & & 20 & $-0,3150$ & 1,2140 & $-0,3360$ & 1,3540 & 0,1110 & 26,1720 & 30,2710 \\
\hline & & 100 & $-0,3160$ & 1,7180 & $-0,3490$ & 1,7960 & 0,1060 & 79,4050 & 88,5540 \\
\hline & 20 & 2 & $-0,0790$ & 0,1100 & $-0,0610$ & 0,1030 & 0,0850 & 0,1610 & 0,1020 \\
\hline & & 20 & $-0,0530$ & 0,0510 & $-0,0590$ & 0,0490 & 0,0370 & 0,0430 & 0,0460 \\
\hline & & 100 & $-0,0500$ & 0,0480 & $-0,0600$ & 0,0540 & 0,0320 & 0,0300 & 0,0410 \\
\hline & 100 & 2 & $-0,0130$ & 0,0590 & $-0,0066$ & 0,0580 & 0,0580 & 0,1070 & 0,0550 \\
\hline & & 20 & $-0,0071$ & 0,0130 & $-0,0059$ & 0,0120 & 0,0120 & 0,0170 & 0,0120 \\
\hline & & 100 & $-0,0074$ & 0,0093 & $-0,0095$ & 0,0083 & 0,0077 & 0,0076 & 0,0079 \\
\hline \multirow[t]{9}{*}{0,8} & 5 & 2 & $-0,0640$ & 0,3540 & $-0,0580$ & 0,3370 & 0,0930 & 16,4330 & 15,1470 \\
\hline & & 20 & $-0,0580$ & 0,1270 & $-0,0610$ & 0,1330 & 0,0480 & 0,2000 & 0,2160 \\
\hline & & 100 & $-0,0680$ & 0,1050 & $-0,0760$ & 0,1180 & 0,0440 & 0,1260 & 0,1930 \\
\hline & 20 & 2 & $-0,0240$ & 0,0730 & $-0,0200$ & 0,0710 & 0,0610 & 0,1210 & 0,0680 \\
\hline & & 20 & $-0,0120$ & 0,0190 & $-0,0130$ & 0,0190 & 0,0160 & 0,0180 & 0,0180 \\
\hline & & 100 & $-0,0098$ & 0,0140 & $-0,0120$ & 0,0140 & 0,0120 & 0,0089 & 0,0130 \\
\hline & 100 & 2 & $-0,0040$ & 0,0540 & $-0,0028$ & 0,0540 & 0,0520 & 0,1020 & 0,0500 \\
\hline & & 20 & $-0,0019$ & 0,0074 & $-0,0016$ & 0,0074 & 0,0072 & 0,0110 & 0,0071 \\
\hline & & 100 & $-0,0015$ & 0,0033 & $-0,0017$ & 0,0033 & 0,0032 & 0,0032 & 0,0032 \\
\hline \multirow[t]{9}{*}{1,9} & 5 & 2 & 0,3580 & 0,9920 & 0,3360 & 0,9210 & 0,1480 & 3,5950 & 3,1260 \\
\hline & & 20 & 0,2680 & 0,4810 & 0,2840 & 0,5570 & 0,1000 & 0,5930 & 0,7550 \\
\hline & & 100 & 0,0072 & 0,0031 & 0,0089 & 0,0031 & 0,0029 & 0,0027 & 0,0030 \\
\hline & 20 & 2 & 0,0690 & 0,1100 & 0,0530 & 0,1030 & 0,0800 & 0,1520 & 0,0950 \\
\hline & & 20 & 0,2680 & 0,4810 & 0,2840 & 0,5570 & 0,1000 & 0,5930 & 0,7550 \\
\hline & & 100 & 0,0410 & 0,0400 & 0,0500 & 0,0440 & 0,0280 & 0,0260 & 0,0360 \\
\hline & 100 & 2 & 0,0049 & 0,0590 & $-0,0005$ & 0,0580 & 0,0570 & 0,1060 & 0,0540 \\
\hline & & 20 & 0,0036 & 0,0120 & 0,0033 & 0,0120 & 0,0110 & 0,0150 & 0,0110 \\
\hline & & 100 & 0,0052 & 0,0079 & 0,0068 & 0,0071 & 0,0069 & 0,0067 & 0,0070 \\
\hline
\end{tabular}


Tabela 3.19: Porcentagem de cobertura (\%) e amplitude (A) dos intervalos de $95 \%$ de confiança para o parâmetro $X_{0}$, para $\sigma_{\delta}^{2}=0,01$.

\begin{tabular}{|c|c|c|c|c|c|c|c|c|c|c|}
\hline \multirow{3}{*}{$X_{0}$} & \multirow{3}{*}{$n$} & \multirow{3}{*}{$k$} & \multicolumn{4}{|c|}{$\sigma_{\epsilon}^{2}=0,04$} & \multicolumn{4}{|c|}{$\sigma_{\epsilon}^{2}=0,4$} \\
\hline & & & \multicolumn{2}{|c|}{ M-usual } & \multicolumn{2}{|c|}{ M-proposto } & \multicolumn{2}{|c|}{ M-usual } & \multicolumn{2}{|c|}{ M-proposto } \\
\hline & & & $\%$ & $\mathrm{~A}$ & $\%$ & A & $\%$ & A & $\%$ & $\mathrm{~A}$ \\
\hline \multirow[t]{9}{*}{0,01} & \multirow[t]{3}{*}{5} & 2 & 92,10 & 0,51 & 91,20 & 0,48 & 84,64 & 1,16 & 83,40 & 1,13 \\
\hline & & 20 & 87,32 & 0,34 & 95,18 & 0,44 & 92,74 & 1,09 & 93,88 & 1,14 \\
\hline & & 100 & 84,50 & 0,32 & 95,19 & 0,44 & 93,60 & 1,10 & 94,92 & 1,16 \\
\hline & \multirow[t]{3}{*}{20} & 2 & 97,46 & 0,43 & 90,12 & 0,33 & 92,90 & 1,02 & 91,52 & 0,98 \\
\hline & & 20 & 91,00 & 0,23 & 94,03 & 0,25 & 93,42 & 0,61 & 93,98 & 0,62 \\
\hline & & 100 & 86,73 & 0,19 & 95,21 & 0,24 & 94,25 & 0,56 & 95,35 & 0,59 \\
\hline & \multirow[t]{3}{*}{100} & 2 & 97,01 & 0,43 & 90,00 & 0,33 & 92,52 & 1,01 & 91,04 & 0,97 \\
\hline & & 20 & 92,34 & 0,23 & 95,26 & 0,25 & 93,68 & 0,61 & 94,50 & 0,62 \\
\hline & & 100 & 85,93 & 0,19 & 94,55 & 0,24 & 93,81 & 0,56 & 94,63 & 0,59 \\
\hline \multirow[t]{9}{*}{0,8} & \multirow[t]{3}{*}{5} & 2 & 94,33 & 0,41 & 89,28 & 0,35 & 84,41 & 0,90 & 82,11 & 0,86 \\
\hline & & 20 & 86,84 & 0,22 & 95,03 & 0,27 & 92,96 & 0,66 & 94,68 & 0,69 \\
\hline & & 100 & 85,65 & 0,19 & 95,56 & 0,27 & 95,35 & 0,64 & 96,35 & 0,67 \\
\hline & \multirow[t]{3}{*}{20} & 2 & 98,35 & 0,39 & 88,44 & 0,27 & 92,70 & 0,91 & 91,18 & 0,86 \\
\hline & & 20 & 93,84 & 0,15 & 94,41 & 0,15 & 94,28 & 0,40 & 94,38 & 0,41 \\
\hline & & 100 & 85,98 & 0,11 & 94,32 & 0,14 & 94,27 & 0,32 & 95,21 & 0,34 \\
\hline & \multirow[t]{3}{*}{100} & 2 & 98,08 & 0,39 & 88,22 & 0,27 & 92,80 & 0,91 & 91,22 & 0,86 \\
\hline & & 20 & 92,75 & 0,15 & 93,96 & 0,15 & 93,66 & 0,40 & 93,76 & 0,41 \\
\hline & & 100 & 87,17 & 0,11 & 94,60 & 0,14 & 94,58 & 0,32 & 95,60 & 0,34 \\
\hline \multirow[t]{9}{*}{1,9} & \multirow[t]{3}{*}{5} & 2 & 92,30 & 0,50 & 90,61 & 0,46 & 84,38 & 1,09 & 82,63 & 1,06 \\
\hline & & 20 & 86,96 & 0,33 & 95,13 & 0,42 & 93,30 & 1,02 & 94,74 & 1,07 \\
\hline & & 100 & 85,84 & 0,30 & 94,73 & 0,42 & 94,05 & 1,04 & 95,36 & 1,10 \\
\hline & \multirow[t]{3}{*}{20} & 2 & 97,61 & 0,43 & 89,92 & 0,32 & 93,28 & 1,00 & 91,90 & 0,96 \\
\hline & & 20 & 91,60 & 0,21 & 94,27 & 0,23 & 93,20 & 0,58 & 93,50 & 0,59 \\
\hline & & 100 & 86,16 & 0,17 & 94,99 & 0,23 & 94,17 & 0,53 & 95,25 & 0,55 \\
\hline & \multirow[t]{3}{*}{100} & 2 & 97,04 & 0,43 & 89,70 & 0,32 & 93,44 & 0,99 & 92,06 & 0,95 \\
\hline & & 20 & 91,21 & 0,21 & 93,85 & 0,23 & 93,78 & 0,58 & 94,04 & 0,58 \\
\hline & & 100 & 87,04 & 0,17 & 95,11 & 0,23 & 94,07 & 0,53 & 95,01 & 0,55 \\
\hline
\end{tabular}


Tabela 3.20: Porcentagem de cobertura (\%) e amplitude (A) dos intervalos de $95 \%$ de confiança para o parâmetro $X_{0}$, para $\sigma_{\delta}^{2}=0,1$.

\begin{tabular}{|c|c|c|c|c|c|c|c|c|c|c|}
\hline \multirow{3}{*}{$X_{0}$} & \multirow{3}{*}{$n$} & \multirow{3}{*}{$k$} & \multicolumn{4}{|c|}{$\sigma_{\epsilon}^{2}=0,04$} & \multicolumn{4}{|c|}{$\sigma_{\epsilon}^{2}=0,4$} \\
\hline & & & \multicolumn{2}{|c|}{ M-usual } & \multicolumn{2}{|c|}{ M-proposto } & \multicolumn{2}{|c|}{ M-usual } & \multicolumn{2}{|c|}{ M-proposto } \\
\hline & & & $\%$ & $\mathrm{~A}$ & $\%$ & A & $\%$ & A & $\%$ & A \\
\hline \multirow[t]{9}{*}{0,01} & 5 & 2 & 95,06 & 1,89 & 92,40 & 1,49 & 96,47 & 2,95 & 95,54 & 2,69 \\
\hline & & 20 & 63,38 & 0,59 & 64,47 & 1,09 & 94,45 & 1,91 & 96,44 & 2,28 \\
\hline & & 100 & 52,69 & 0,48 & 89,24 & 1,18 & 93,77 & 3,66 & 97,34 & 3,68 \\
\hline & 20 & 2 & 99,87 & 1,17 & 96,27 & 0,71 & 97,90 & 1,49 & 90,92 & 1,13 \\
\hline & & 20 & 92,76 & 0,48 & 94,48 & 0,52 & 94,24 & 0,78 & 95,84 & 0,81 \\
\hline & & 100 & 70,38 & 0,30 & 90,35 & 0,51 & 91,43 & 0,63 & 96,91 & 0,77 \\
\hline & 100 & 2 & 100,00 & 0,98 & 93,41 & 0,40 & 99,37 & 1,28 & 92,63 & 0,90 \\
\hline & & 20 & 99,77 & 0,36 & 95,09 & 0,24 & 96,96 & 0,50 & 94,26 & 0,43 \\
\hline & & 100 & 92,70 & 0,21 & 94,42 & 0,23 & 92,83 & 0,34 & 95,11 & 0,35 \\
\hline \multirow[t]{9}{*}{0,8} & 5 & 2 & 98,77 & 1,31 & 97,54 & 0,98 & 95,50 & 1,78 & 92,22 & 1,56 \\
\hline & & 20 & 61,85 & 0,34 & 81,25 & 0,69 & 91,31 & 0,94 & 97,20 & 1,13 \\
\hline & & 100 & 49,29 & 0,27 & 87,82 & 0,69 & 91,83 & 0,89 & 98,03 & 1,12 \\
\hline & 20 & 2 & 100,00 & 1,03 & 94,09 & 0,53 & 98,96 & 1,33 & 90,35 & 0,96 \\
\hline & & 20 & 94,44 & 0,32 & 95,11 & 0,32 & 94,86 & 0,51 & 95,41 & 0,52 \\
\hline & & 100 & 70,38 & 0,17 & 92,41 & 0,31 & 88,72 & 0,36 & 95,38 & 0,45 \\
\hline & 100 & 2 & 100,00 & 0,95 & 92,25 & 0,34 & 99,11 & 1,25 & 91,92 & 0,86 \\
\hline & & 20 & 99,89 & 0,30 & 94,89 & 0,16 & 98,14 & 0,41 & 94,17 & 0,33 \\
\hline & & 100 & 95,00 & 0,14 & 94,88 & 0,14 & 94,63 & 0,22 & 94,77 & 0,22 \\
\hline \multirow[t]{9}{*}{1,9} & 5 & 2 & 96,26 & 1,78 & 92,14 & 1,45 & 96,02 & 2,54 & 93,51 & 2,27 \\
\hline & & 20 & 63,58 & 0,56 & 67,24 & 1,03 & 93,41 & 1,60 & 96,51 & 1,90 \\
\hline & & 100 & 49,30 & 0,46 & 83,75 & 1,21 & 93,71 & 1,54 & 97,19 & 1,91 \\
\hline & 20 & 2 & 100,00 & 1,17 & 96,27 & 0,70 & 98,36 & 1,48 & 92,10 & 1,13 \\
\hline & & 20 & 94,26 & 0,46 & 94,95 & 0,49 & 93,99 & 0,74 & 95,62 & 0,77 \\
\hline & & 100 & 72,44 & 0,28 & 94,18 & 0,48 & 90,11 & 0,60 & 96,40 & 0,72 \\
\hline & 100 & 2 & 100,00 & 0,97 & 94,27 & 0,39 & 99,07 & 1,27 & 92,15 & 0,89 \\
\hline & & 20 & 99,59 & 0,35 & 95,18 & 0,23 & 97,48 & 0,48 & 94,33 & 0,41 \\
\hline & & 100 & 93,38 & 0,20 & 94,98 & 0,21 & 92,91 & 0,32 & 94,91 & 0,33 \\
\hline
\end{tabular}




\section{Comparação dos métodos de}

\section{cálculo de incerteza expandida}

Muitos termos utilizados na metrologia, não estão difundidos entre os estatísticos, por exemplo, mensurando, valor do mensurando, erro e incerteza. Segundo o Vocabulário Internacional de Metrologia Iso (VIM, 1993) define-se mensurando ao atributo sujeito à medição de um fenômeno, corpo ou substância. Em termos estatísticos mensurando (ou grandeza) é equivalente à definição de variável aleatória e o valor do mensurando referese ao parâmetro populacional que é uma quantidade desconhecida. Termos como erro e incerteza devem ser distingüidos, pois têm conceitos diferentes. O erro de medição é a diferença entre um resultado individual da medição e o valor do mensurando. Desde que o valor do mensurando é uma quantidade desconhecida, então, o erro de medição também, será desconhecido. Segundo VIM (1993) e INMETRO (1995), a incerteza de medição é um parâmetro associado ao resultado, que caracteriza a dispersão dos valores obtidos em torno da média. O parâmetro pode ser um desvio padrão ou um múltiplo como, por exemplo, o comprimento de um intervalo de confiança.

Nesta seção apresentamos algumas terminologias e métodos, existentes na literatura estatística, referentes à avaliação e expressão da incerteza de medição. Também, mediante um 
estudo de simulação observamos o comportamento das incertezas fornecidas pelos métodos. Particularmente, esses métodos servem para estimar as incertezas das concentrações das soluções-padrão.

\subsection{Lei de propagação de incertezas}

Suponha que um processo possa ser descrito como uma função $f$ de um conjunto de variáveis aleatórias, denominadas variáveis de entrada. A relação entre as variáveis de entrada e o mensurando $Z$ é representada por:

$$
Z=f\left(W_{1}, W_{2}, \cdots, W_{m}\right)
$$

O valor do mensurando $\eta$ é a avaliação da função $f$ nos parâmetros $\mu_{i}=E\left(W_{i}\right), i=$ $1, \cdots, m$.

O desvio padrão da variável aleatória $W_{i}$ é denominado pelo Comitê Internacional de Pesos e Medidas (CIPM) de incerteza padrão e a sua estimativa é representada por $u\left(W_{i}\right)$. O guia ISOGUM categoriza as incertezas padrão em dois tipos:

Incerteza tipo A: a avaliação é feita por meio de uma análise estatística clássica baseada numa série de medições.

Incerteza tipo B: a avaliação é feita com base no conhecimento disponível, experiência do cientista ou da documentação existente. As fontes de informação podem ser:

- certificado de calibração,

- normas, manuais de instrumento de medição ou literatura,

- medições anteriores,

- conhecimento do técnico sobre as características do sistema de medição.

Os dois tipos de incerteza estão baseados em distribuições de probabilidade. A diferença é 
que no tipo A, a incerteza é estimada baseando-se nas medições repetidas, enquanto que no tipo B, a distribuição é baseada na experiência ou informação externa ao metrólogo.

O desvio padrão do mensurando $Z$ é denominado incerteza padrão combinada (ou incerteza combinada) e sua estimativa é representada por $u_{c}(Z)$. A incerteza combinada é função das incertezas padrão $u\left(W_{i}\right)$ que podem ser do tipo A ou B.

O produto da incerteza padrão combinada por um fator de abrangência $k$ é denominado de incerteza expandida e é representado por $U(Z)=k u_{c}(Z)$. A incerteza expandida define um intervalo em torno do resultado de medição com probabilidade de abrangência $p$ (Kyriasis, 2004). O guia ISOGUM utiliza o termo nível de confiança como um sinônimo para probabilidade de abrangência, mas a literatura de metrologia recomenda utilizar essa última denominação, já que a incerteza padrão combinada pode ser função de incertezas do tipo B. Em termos estatísticos, se a incerteza padrão combinada depender só de incertezas do tipo A, então a incerteza expandida define um intervalo de confiança.

A seguir apresentamos uma forma de combinar incertezas padrão para a obtenção da incerteza padrão combinada.

$\mathrm{Na}$ equação (4.1), em geral, a função $f$ não é linear. Se $f$ e as primeiras derivadas parciais em relação a $W_{i}$ em torno dos parâmetros $\mu_{i}, i=1, \cdots, m$, são contínuas, então, podemos obter por meio de expansão em série de Taylor até a primeira ordem ao redor da média populacional $\mu=\left(\mu_{1}, \mu_{2}, \cdots, \mu_{m}\right)$ uma aproximação para $Z$

$$
Z \approx f(\mu)+\sum_{i=1}^{m}\left(\left.\frac{\partial f}{\partial W_{i}}\right|_{W=\mu}\right)\left(W_{i}-\mu_{i}\right) .
$$

A partir da equação (4.2) temos uma aproximação para a média e a variância de $Z$ dadas por

$$
E(Z) \approx f(\mu)
$$

e

$$
E\left[(Z-f(\mu))^{2}\right] \approx E\left[\left(\sum_{i=1}^{m}\left(\left.\frac{\partial f}{\partial W_{i}}\right|_{W=\mu}\right)\left(W_{i}-\mu_{i}\right)\right)^{2}\right]
$$


Logo, a variância de $Z$, denotada por $\sigma_{Z}^{2}$ é dada por

$$
\begin{gathered}
\sigma_{Z}^{2} \approx E\left[\sum_{i=1}^{m}\left(\left.\frac{\partial f}{\partial W_{i}}\right|_{W=\mu}\right)^{2}\left(W_{i}-\mu_{i}\right)^{2}+\left.\left.2 \sum_{i=1}^{m-1} \sum_{j=i+1}^{m} \frac{\partial f}{\partial W_{i}}\right|_{W=\mu} \frac{\partial f}{\partial W_{j}}\right|_{W=\mu}\left(W_{i}-\mu_{i}\right)\left(W_{j}-\mu_{j}\right)\right] \\
=\sum_{i=1}^{m}\left(\left.\frac{\partial f}{\partial W_{i}}\right|_{W=\mu}\right)^{2} \sigma_{W_{i}}^{2}+\left.\left.2 \sum_{i=1}^{m-1} \sum_{j=i+1}^{m} \frac{\partial f}{\partial W_{i}}\right|_{W=\mu} \frac{\partial f}{\partial W_{j}}\right|_{W=\mu} \operatorname{cov}\left(W_{i}, W_{j}\right)
\end{gathered}
$$

em que $\sigma_{W_{i}}^{2}$ é a variância da variável $W_{i}$ e $\operatorname{cov}\left(W_{i}, W_{j}\right)$ é a covariância entre as variáveis $W_{i}$ e $W_{j}$. No caso em que as variáveis $W_{i}$ são independentes, a expressão (4.5) se reduz a

$$
\sigma_{Z}^{2} \approx \sum_{i=1}^{m}\left(\left.\frac{\partial f}{\partial W_{i}}\right|_{W=\mu}\right)^{2} \sigma_{W_{i}}^{2},
$$

sendo as derivadas parciais, $\left.\frac{\partial f}{\partial W_{i}}\right|_{W=\mu}$, chamadas de coeficientes de sensibilidade. $\mathrm{O}$ quadrado da estimativa da incerteza padrão de $W_{i}, u\left(W_{i}\right)$, é uma estimativa de $\sigma_{W_{i}}^{2}$ EURACHEM/ CITAC(2002). Assim, a expressão (4.6) é estimada por

$$
u_{c}(Z)^{2}=\sum_{i=1}^{m} \lambda_{i} u\left(W_{i}\right)^{2},
$$

em que $\lambda_{i}=\left(\left.\frac{\partial f}{\partial W_{i}}\right|_{W=\mu}\right)^{2}$.

A equação (4.7) é conhecida como lei de propagação de incertezas.

\subsubsection{Exemplo}

Exemplificamos a utilidade da Lei de propagação de incertezas no cálculo da incerteza expandida da concentração das soluções-padrão.

Na Seção 3.2 , vimos que, a concentração fixada $c_{i 2}$ é função de $c_{0}, p_{0}, p_{i}, b_{0}$ e $b_{i}$ que, também, são quantidades fixadas. No entanto, devido aos erros cometidos no processo de preparação não se consegue obter exatamente as quantidades fixadas. Portanto temos que no processo, a concentração certificada $\left(C_{0}\right)$, volume da primeira e segunda pipetagem $\left(P_{0} \mathrm{e}\right.$ $P_{i}$, respectivamente) e o volume do primeiro e segundo balão $\left(P_{0}\right.$ e $P_{i}$, respectivamente) são variáveis aleatórias e conseqüentemente a concentração da solução-padrão também, logo:

$$
C_{i 2}=\frac{C_{0} P_{0} P_{i}}{B_{0} B_{i}}
$$


em que as letras maiúsculas indicam variáveis aleatórias tais que $E\left(C_{i 2}\right)=c_{i 2}, E\left(C_{0}\right)=$ $c_{0}, E\left(P_{0}\right)=p_{0}, E\left(P_{i}\right)=p_{i}, E\left(B_{o}\right)=b_{0}, E\left(B_{i}\right)=b_{i}$.

Seja $W=\left(C_{0}, P_{0}, P_{i}, B_{0}, B_{i}\right)$ e $\mu=\left(c_{0}, p_{0}, p_{i}, b_{0}, b_{i}\right)$. Pelos resultados apresentados na Seção 4.1 temos que uma aproximação para a concentração da solução-padrão é dada por

$$
\begin{aligned}
C_{2 i}= & \frac{c_{0} p_{0} p_{i}}{b_{0} b_{i}}+\left(\left.\frac{\partial C_{2 i}}{\partial C_{0}}\right|_{W=\mu}\right)\left(C_{0}-c_{0}\right)+\left(\left.\frac{\partial C_{2 i}}{\partial P_{0}}\right|_{W=\mu}\right)\left(P_{0}-p_{0}\right) \\
& +\left(\left.\frac{\partial C_{2 i}}{\partial P_{i}}\right|_{W=\mu}\right)\left(P_{i}-p_{i}\right)+\left(\left.\frac{\partial C_{2 i}}{\partial B_{0}}\right|_{W=\mu}\right)\left(B_{0}-b_{0}\right)+\left(\left.\frac{\partial C_{2 i}}{\partial B_{i}}\right|_{W=\mu}\right)\left(B_{i}-b_{i}\right) .
\end{aligned}
$$

Considerando a independência entre as variáveis $C_{0}, P_{0}, P_{i}, B_{0}$ e $B_{i}$, a variância de $C_{2 i}$ é dada por

$$
\begin{aligned}
\sigma_{C_{2 i}}^{2}= & \left(\left.\frac{\partial C_{2 i}}{\partial C_{0}}\right|_{W=\mu}\right)^{2} \sigma_{C_{0}}^{2}+\left(\left.\frac{\partial C_{2 i}}{\partial P_{0}}\right|_{W=\mu}\right)^{2} \sigma_{P_{0}}^{2}+\left(\left.\frac{\partial C_{2 i}}{\partial P_{1}}\right|_{W=\mu}\right)^{2} \sigma_{P_{1}}^{2} \\
& +\left(\left.\frac{\partial C_{2 i}}{\partial B_{0}}\right|_{W=\mu}\right)^{2} \sigma_{B_{0}}^{2}+\left(\left.\frac{\partial C_{2 i}}{\partial B_{i}}\right|_{W=\mu}\right)^{2} \sigma_{B_{i}}^{2},
\end{aligned}
$$

em que $\sigma_{C_{0}}^{2}, \sigma_{P_{0}}^{2}, \sigma_{P_{i}}^{2}, \sigma_{B_{0}}^{2}$ e $\sigma_{B_{i}}^{2}$ são as variâncias de $C_{0}, P_{0}, P_{i}, B_{0}$ e $B_{i}$, respectivamente. Logo,

$$
\sigma_{C_{2}}^{2}=\lambda_{C_{0}} \sigma_{C_{0}}^{2}+\lambda_{P_{0}} \sigma_{P_{0}}^{2}+\lambda_{P_{i}} \sigma_{P_{i}}^{2}+\lambda_{B_{0}} \sigma_{B_{0}}^{2}+\lambda_{B_{i}} \sigma_{B_{i}}^{2}
$$

em que $\sqrt{\lambda_{C_{0}}}, \sqrt{\lambda_{P_{0}}}, \sqrt{\lambda_{P_{i}}}, \sqrt{\lambda_{B_{0}}}, \sqrt{\lambda_{B_{1}}}$ são os coeficientes de sensibilidade.

Segundo a lei de propagação de incertezas temos que a equação (4.9) é estimada por

$$
u_{c}\left(C_{2}\right)^{2}=\lambda_{C_{0}} u\left(C_{0}\right)^{2}+\lambda_{P_{0}} u\left(P_{0}\right)^{2}+\lambda_{P_{i}} u\left(P_{i}\right)^{2}+\lambda_{B_{0}} u\left(B_{0}\right)^{2}+\lambda_{B_{i}} u\left(B_{i}\right)^{2}
$$

em que $u\left(C_{0}\right), u\left(P_{0}\right), u\left(P_{i}\right), u\left(B_{0}\right)$ e $u\left(B_{i}\right)$ são as incertezas padrão de $C_{0}, P_{0}, P_{i}, B_{0}$ e $B_{i}$, respectivamente.

\subsection{Métodos para o cálculo de incertezas expandi- das}

Um dos problemas na metrologia é obter um intervalo de confiança para o valor do mensurando (parâmetro populacional $\eta$ ), associado a um mensurando (variável dependente) $Z$, 
que é função de variáveis independentes $W_{i}, i=1, \cdots, m$, ou seja,

$$
Z=f\left(W_{1}, W_{2}, \cdots, W_{m}\right)
$$

Em geral, $f$ não é linear, e não é fácil conseguir analiticamente a distribuição exata de $Z$. Neste caso, como vimos na Seção 4.1, a partir de uma expansão em série de Taylor até primeira ordem em torno da média $\mu=\left(\mu_{1}, \mu_{2}, \cdots, \mu_{m}\right)$, sendo $\mu_{i}$ a média da variável $W_{i}$, temos uma aproximação para $Z$ e para sua variância, dadas em (4.2) e (4.6), respectivamente.

A aproximação (4.2) é uma combinação linear das variáves $\left(W_{i}-\mu_{i}\right), i=1, \cdots, m$. No caso em que todas as variáveis $W_{i}$ têm distribuição normal então a variável $Z$ tem distribuição aproximadamente normal. É comun que algumas variáveis forneçam incertezas do tipo A e outras do tipo B. O guia ISOGUM recomenda supor distribuição uniforme ou triangular para as variáveis associadas às incertezas do tipo B. Ainda assim, temos que $Z$ tem distribuição aproximadamente normal, pois a soma de variáveis com distribuição uniforme (e/ou triangular) é aproximadamente normal (Dietrich, 1991) e segundo o teorema de limite central a soma de variáveis associadas as incertezas do tipo A, também pode ser aproximada uma distribuição normal.

Apesar da distribuição da variável aleatória $Z=f\left(W_{1}, W_{2}, \cdots, W_{m}\right)$ ser aproximadamente normal apenas quando o número de variáveis do tipo A e ou do tipo B são grandes, os métodos desenvolvidos a partir de uma variável $Z$ com distribuição normal, que apresentamos a seguir, são utilizados, na metrologia, para obter incerteza expandida.

Seja $Z$ uma variável aleatória com distribuição normal com média $\eta$ e variância $\sigma_{Z}^{2}=$ $\sum_{i=1}^{m} \lambda_{i} \sigma_{i}^{2}$, em que $\lambda_{i}$ e $\sigma_{i}^{2}, i=1, \cdots, m$, são, respectivamente, constantes conhecidas e variâncias desconhecidas. Para obter um intervalo de confiança para o parâmetro $\eta$, é necessário encontrar uma função $h\left(S_{1}^{2}, S_{2}^{2}, \cdots, S_{m}^{2}\right)$, sendo $S_{i}^{2}$ um estimador não viesado de $\sigma_{i}^{2}$, tal que, para um nível de confiança $p$ se tenha

$$
P\left[(Z-\eta) \leq h\left(S_{1}^{2}, S_{2}^{2}, \cdots, S_{m}^{2}, p\right)\right]=p
$$

A solução de (9), para $m=2$, inicialmente foi proposta por Behrens (1929) e logo for- 
malizada por Fisher $(1935$, 1941) e Jeffreys (1940). Para resolver esse problema Jeffreys introduziu uma função de distribuição a priori para o desvio padrão desconhecido, $\sigma_{i}$. Essa solução encontrada sob o ponto de vista Bayessiano é igual ao resultado obtido por Fisher. A metodologia de Fisher dá validade ao uso da integral de Behrens para obter o limite fiducial para a diferença de médias de duas populações normais quando não se tem informação acerca das variâncias dessas populações.

Por outro lado Welch (1947 (a) e 1947 (b)) propõem duas metodologias para a solução de (9), baseadas na teoria freqüentista. No primeiro método, uma solução numérica é obtida aplicando uma solução em série de Taylor. O outro método (recomendado pelo guia ISOGUM) baseia-se na distribuição aproximada de $\sum_{i=1}^{m} \lambda_{i} S_{i}^{2}$. Só estamos interessados em estudar os métodos clássicos propostos por Welch.

\subsubsection{Incerteza expandida obtida por meio da solução em séries}

Welch (1947 (a)) e Aspin (1948) utilizando expansão em série de Taylor encontraram uma solução numérica para a equação (9). Detalhes da demonstração encontram-se no Apêndice 2.

Welch considera a solução para a equação (9) definindo a função $h\left(S_{1}^{2}, S_{2}^{2}, \cdots, S_{m}^{2}, p\right)$ como

$$
\begin{aligned}
h\left(S_{1}^{2}, S_{2}^{2}, \cdots, S_{m}^{2}, p\right) & =h_{0}\left(S_{1}^{2}, S_{2}^{2}, \cdots, S_{m}^{2}, p\right)+h_{1}\left(S_{1}^{2}, S_{2}^{2}, \cdots, S_{m}^{2}, p\right) \\
& +h_{2}\left(S_{1}^{2}, S_{2}^{2}, \cdots, S_{m}^{2}, p\right)+\cdots
\end{aligned}
$$


em que

$$
\begin{aligned}
& h_{0}(\mathbf{V})=\xi \sqrt{\sum_{i=1}^{m} \lambda_{i} S_{i}^{2}}, \\
& \frac{h_{1}(\mathbf{V})}{h_{0}(\mathbf{V})}=\frac{1}{4}\left(1+\xi^{2}\right) V_{21} \text {, } \\
& \frac{h_{2}(\mathbf{V})}{h_{0}(\mathbf{V})}=\left[-\frac{1}{2}\left(1+\xi^{2}\right) V_{22}+\frac{1}{3}\left(3+5 \xi^{2}+\xi^{4}\right) V_{32}-\frac{1}{32}\left(15+32 \xi^{2}+9 \xi^{4}\right) V_{21}^{2}\right] \text {, } \\
& \frac{h_{3}(\mathbf{V})}{h_{0}(\mathbf{V})}=\left[\left(1+\xi^{2}\right) V_{23}-2\left(3+5 \xi^{2}+\xi^{4}\right) V_{33}-\frac{1}{3}\left(15+32 \xi^{2}+9 \xi^{4}\right) V_{22} V_{21}\right. \\
& +\frac{1}{8}\left(75+173 \xi^{2}+63 \xi^{4}+5 \xi^{6}\right) V_{43}-\frac{1}{12}\left(105+298 \xi^{2}+140 \xi^{4}+15 \xi^{6}\right) V_{32} V_{21} \\
& \left.+\frac{1}{384}\left(945+3169 \xi^{2}+1811 \xi^{4}+243 \xi^{6}\right) V_{21}^{3}\right] \text {, } \\
& \frac{h_{4}(\mathrm{~V})}{h_{0}(\mathrm{~V})}=\left[-2\left(1+\xi^{2}\right) V_{24}+\frac{28}{3}\left(3+5 \xi^{2}+\xi^{4}\right) V_{34}\right. \\
& -\frac{1}{4}\left(15+32 \xi^{2}+9 \xi^{4}\right)\left\{V_{23} V_{21}+\frac{1}{2} V_{22}^{2}\right\} \\
& -\frac{3}{2}\left(75+173 \xi^{2}+63 \xi^{4}+5 \xi^{6}\right) V_{44} \\
& +\frac{1}{2}\left(105+298 \xi^{2}+140 \xi^{4}+15 \xi^{6}\right)\left\{\frac{1}{3} V_{22} V_{32}+V_{21} V_{33}\right\} \\
& +\frac{1}{4}\left(15+33 \xi^{2}+11 \xi^{4}+\xi^{6}\right) V_{44} \\
& +\frac{1}{5}\left(735+2170 \xi^{2}+1126 \xi^{4}+168 \xi^{6}+7 \xi^{8}\right) V_{54} \\
& -\frac{1}{64}\left(945+3169 \xi^{2}+1811 \xi^{4}+243 \xi^{6}\right) V_{42} V_{21}^{2} \\
& -\frac{1}{18}\left(945+3354 \xi^{2}+2166 \xi^{4}+425 \xi^{6}+25 \xi^{8}\right) V_{32}^{2} \\
& -\frac{1}{32}\left(4725+16586 \xi^{2}+10514 \xi^{4}+1974 \xi^{6}+105 \xi^{8}\right) V_{21} V_{43} \\
& +\frac{1}{96}\left(10395+42429 \xi^{2}+31938 \xi^{4}+7335 \xi^{6}+495 \xi^{8}\right) V_{32} V_{21}^{2} \\
& \left.-\frac{1}{6144}\left(135135+626144 \xi^{2}+542026 \xi^{4}+145320 \xi^{6}+11583 \xi^{8}\right) V_{21}^{4}\right] \text {, } \\
& \mathrm{V}=\left(S_{1}^{2}, S_{2}^{2}, \cdots, S_{m}^{2}, p\right)
\end{aligned}
$$

e $\xi$ é o quantil $\left(1-\frac{\alpha}{2}\right)$ da distribuição normal padrão, sendo $\alpha=1-p$, e

$$
V_{r u}=\frac{\left(\sum_{i=1}^{m} \frac{\lambda_{i}^{r} S_{i}^{2 r}}{f_{i}^{u}}\right)}{\left(\sum_{i=1}^{m} \lambda_{i} S_{i}^{2}\right)^{r}}, r=2, \cdots, 5 \text { e } u=1, \cdots, 4 .
$$




\subsubsection{Incerteza expandida recomendada pelo guia ISOGUM}

Reescrevendo (9) como

$$
P\left[\frac{Z-\eta}{\sqrt{\sum_{i=1}^{m} \lambda_{i} S_{i}^{2}}} \leq \frac{h\left(S_{1}^{2}, S_{2}^{2}, \cdots, S_{m}^{2}, p\right)}{\sqrt{\sum_{i=1}^{m} \lambda_{i} S_{i}^{2}}}\right]=p
$$

o problema se reduz em encontrar a distribuição da variável aleatória

$$
\frac{Z-\eta}{\sqrt{\sum_{i=1}^{m} \lambda_{i} S_{i}^{2}}}
$$

Considerando que $\sum_{i=1}^{k} \lambda_{i} S_{i}^{2}=\sum_{i=1}^{k} a_{i} \chi_{i}^{2}$, em que $a_{i}=\frac{\lambda_{i} \sigma_{i}^{2}}{f_{i}}$ e $\chi_{i}^{2}=\frac{f_{i}}{\sigma_{i}^{2}} S_{i}^{2}$, e que a distribuição de $\chi_{i}^{2}$ pode ser aproximada por uma curva do tipo III de Pearson, Welch (1947(a)) provou que a variável aleatória (4.18) segue uma distribuição t-Student com $f$ graus de liberdade, em que

$$
f=\frac{\left(\sum_{i=1}^{m} \lambda_{i} \sigma_{i}^{2}\right)^{2}}{\sum_{i=1}^{m} \frac{1}{f_{i}}\left(\lambda_{i} \sigma_{i}^{2}\right)^{2}} .
$$

Os detalhes da demonstração encontram-se no Apêndice 3.

Fairfield-Smith (1936) denomina a equação (4.19) de fórmula de Welch-Satterthwaite.

Como (4.6) é aproximada por (4.7), temos uma aproximação para os graus de liberdade (4.19), chamado pelo guia ISOGUM de graus de liberdade efetivos. sendo essa aproximação dada por

$$
f_{e}=\frac{\left(\sum_{i=1}^{m} \lambda_{i} u_{i}^{2}\right)^{2}}{\sum_{i=1}^{m} \frac{1}{f_{i}}\left(\lambda_{i} u_{i}^{2}\right)^{2}} .
$$

Assim, a incerteza expandida para $Z$ é definida como:

$$
U(Z)=t_{f_{e}, p} u_{c}(Z)
$$

em que $t_{f_{e}, p}$ é o quantil $\frac{(1-p)}{2}$ da distribuição t-Student com $f_{e}$ graus de liberdade e $u_{c}(z)=$ $\sqrt{\sum_{i=1}^{m} \lambda_{i} u_{i}^{2}}$ é a incerteza combinada.

O intervalo $\left(-t_{f_{e}, p} u_{c}(Z) ; t_{f_{e}, p} u_{c}(Z)\right)$ é conhecido como intervalo de abrangência, por estar em função da incerteza combinada. 
Embora esse intervalo, obtido utilizando-se métodos clássicos, seja aplicado na prática, existe muita discussão entre os metrólogos pois a incerteza combinada utiliza métodos estatísticos clássicos e Bayessianos.

\subsection{Comparação dos métodos para obter as in- certezas expandidas}

Para comparar os métodos de cálculo de incertezas expandidas apresentados na Seção 4.1, vamos supor que $Z-\eta=W_{A}+W_{B}$, em que $W_{A}$ e $W_{B}$ são variáveis aleatórias independentes. A variável $W_{A}$, associada à incerteza do tipo $\mathrm{A}$, tem uma distribuição normal com média 0 e variância $\sigma^{2}$, e $W_{B}$, associada à incerteza do tipo $\mathrm{B}$, provém de uma distribuição normal padrão.

Denotamos por $W S$, a incerteza expandida recomendada pelo guia ISOGUM dada em (4.21).

Utilizando o método de solução em séries obtemos as incertezas expandidas

$$
\begin{aligned}
& H 2=h_{0}+h_{1}+h_{2}, \\
& H 3=h_{0}+h_{1}+h_{2}+h_{3}, \\
& H 4=h_{0}+h_{1}+h_{2}+h_{3}+h_{4},
\end{aligned}
$$

em que $h_{0}, h_{1}, h_{2}, h_{3}$, e $h_{4}$ são dadas em (4.13), (4.14), (4.15), (4.16), e (4.17), respectivamente.

Além disso, considerando $\sigma^{2}$ conhecido e como o desvio padrão de $Z$ é $\sqrt{1+\sigma^{2}}$ temos que o erro do intervalo é $V=\xi \sqrt{1+\sigma^{2}}$.

Foram geradas 500 amostras de tamanho $n_{A}$ da distribuição normal com média $0 \mathrm{e}$ variância $\sigma^{2}$ e de tamanho $n_{B}$ da distribuição normal padrão. Os valores considerados 
foram $n_{A}=3$ e $100, n_{B}=3$ e 100 e $\sigma^{2}=0,01 ; 0,20 ; 0,39 ; \cdots ; 3$. Para cada amostra foram calculadas as estimativas das incertezas expandidas $W S, H 2, H 3, H 4$ considerando uma probabilidade de abrangência de $95 \%$. As Figuras 4.1 a 4.4 apresentam os gráficos da média das 500 incertezas expandidas $W S, H 2, H 3, H 4$ e a o intervalo $V$ em função do desvio padrão $\sigma$.

Analisando as Figuras 4.1 a 4.3 observamos que os valores das incertezas expandidas são maiores do que os valores de $V$ para todo $\sigma$. Isso era esperado pois, apenas, $V$ supõe que a variância $\sigma^{2}$ é conhecida. Já na Figura 4.4, quando $n_{A}=100$ e $n_{B}=100$, observamos que todas as incertezas expandidas são próximas do valor de $V$ para todo valor de $\sigma$.

Nas Figuras 4.1, 4.2 e 4.3 a incerteza expandida $H 4$ apresenta valores bem maiores do que as demais para alguns valores de $\sigma$. Nas Figuras 4.1 e 4.3, verificamos que, entre as incertezas expandidas, $H 2$ é a que apresenta os menores valores para $\sigma \leq 0,5$. Quando $\sigma \geq 0,5$ os menores valores são dados por $W S$, sendo que na Figura 4.3 todas as incertezas expandidas, inclusive $V$, são próximas quando $\sigma \geq 2$.

Observamos que, em geral $W S<H 2<H 3<H 4$. Logo, se a cobertura dos intervalos obtidos a partir dessas incertezas estiverem próximas de $p$, então $W S$ poderia ser considerado a melhor aproximação.

Ballico (2000) fez a comparação de $W S, H 2$ e $H 3$, além dessas incertezas expandidas considerou, também, o intervalo "fiducial"de Fisher-Behrens (Fisher, 1941). Considerando a incerteza expandida $H 3$ como a melhor aproximação e concluiu que a fórmula de WelchSatterthwaite subestima a incerteza expandida e portanto não fornece uma boa aproximação. 


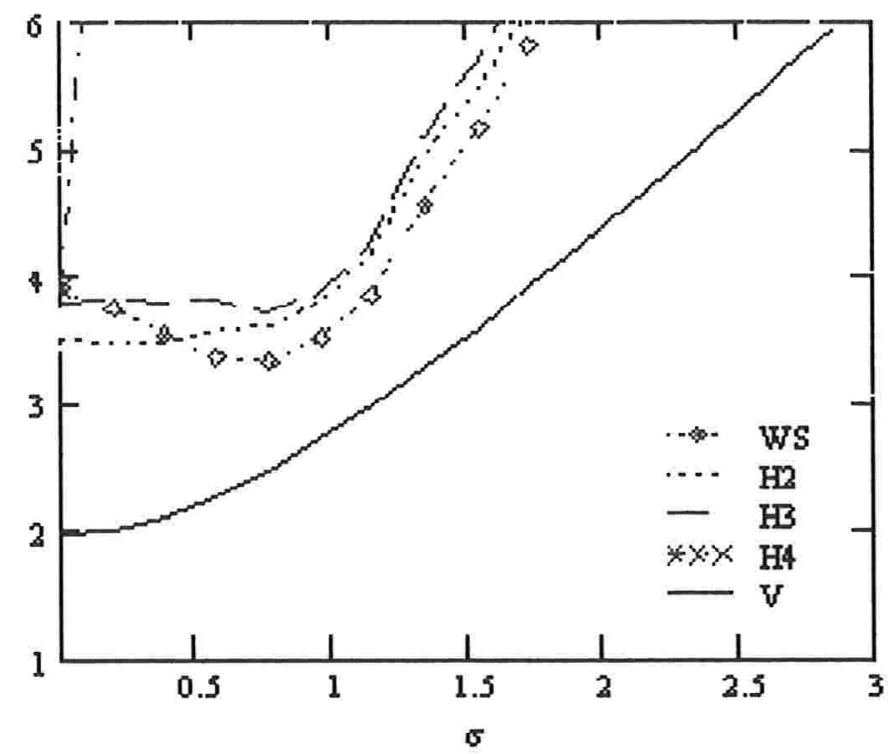

Figura 4.1: Incertezas expandidas quando $n_{A}=3$ e $n_{B}=3$ em função do desvio padrão $\sigma$.

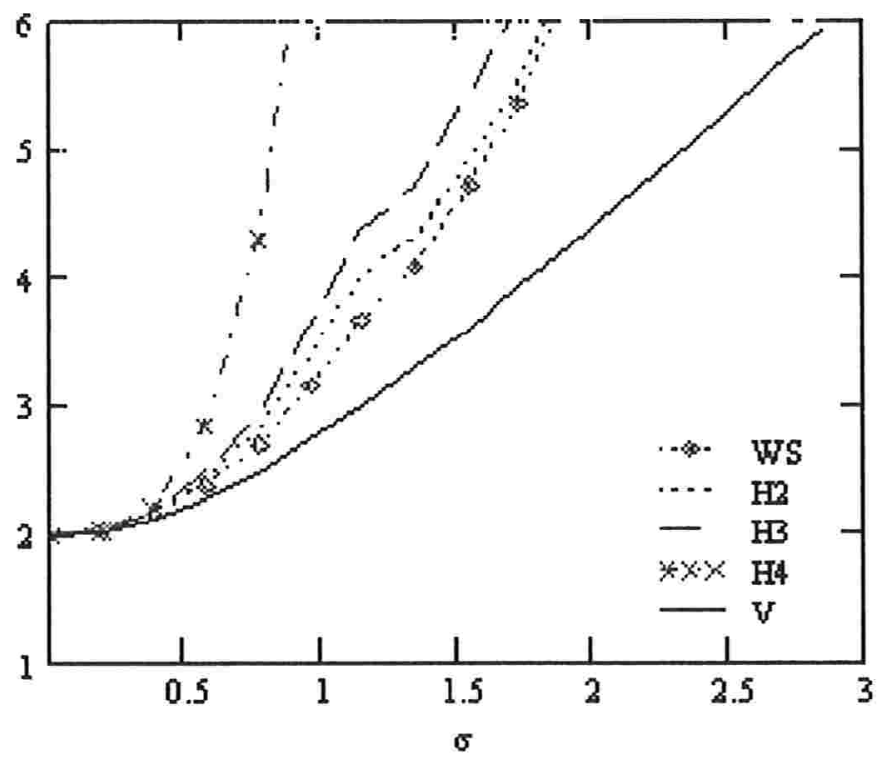

Figura 4.2: Incertezas expandidas quando $n_{A}=3$ e $n_{B}=100$ em função do desvio padrão $\sigma$. 


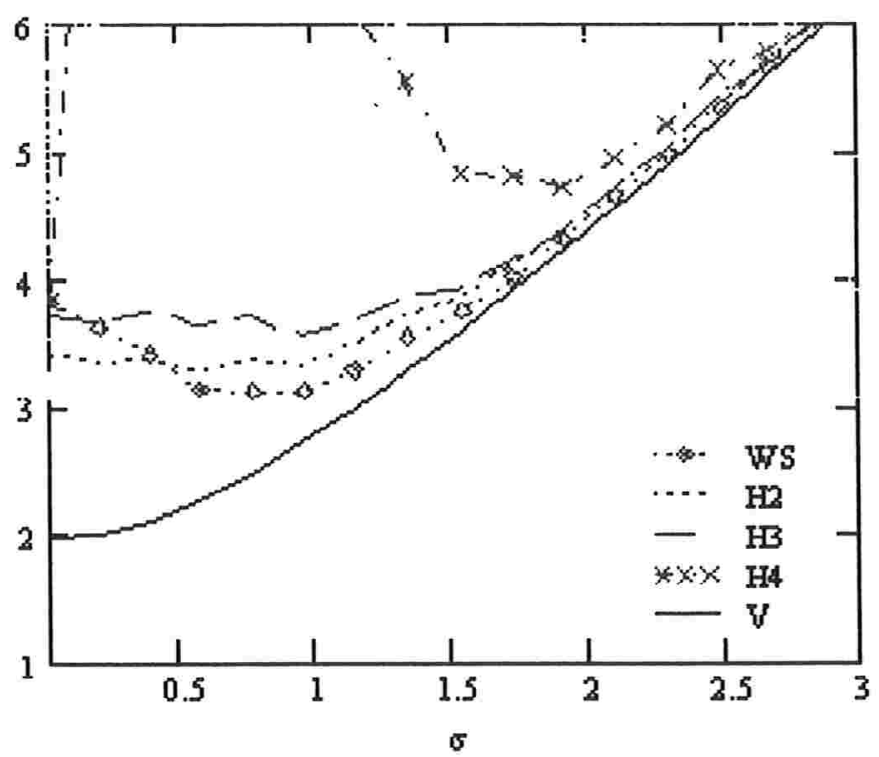

Figura 4.3: Incertezas expandidas quando $n_{A}=100$ e $n_{B}=3$ em função do desvio padrão $\sigma$.

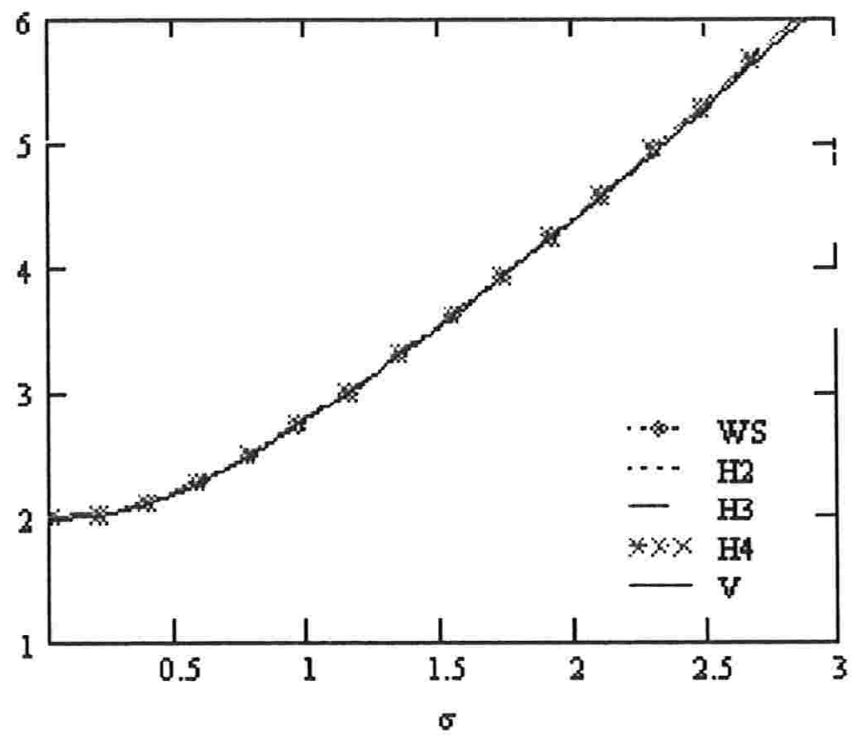

Figura 4.4: Incertezas expandidas quando $n_{A}=100$ e $n_{B}=100$ em função do desvio padrão $\sigma$. 


\section{CAPÍtulo 5}

\section{Aplicações}

Neste capítulo, aplicamos os resultados apresentados nos Capítulos 2, 3 e 4 a conjuntos de dados obtidos no laboratório de química do IPT e do Instituto Adolfo Lutz.

\subsection{Aplicação do cálculo de incerteza}

Nesta seção, mostramos uma aplicação da lei de propagação de erros e dos métodos de cálculo de incerteza expandida para obter as incertezas associadas à concentração das soluções-padrão. Os dados utilizados se referem ao elemento alumínio e foram fornecidos pelo Instituto Adolfo Lutz.

Como vimos na Seção 3.2 , a concentração $c_{2 i}$, da $i$-ésima solução-padrão é função da concentração certificada $c_{0}$, do volume da primeira pipetagem $p_{0}$, do volume do balão $b_{0}$, do volume da segunda pipetagem $p_{i}$ e do volume do balão $b_{i}$.

Para preparar 7 soluções-padrão com as seguintes concentrações:

$$
4,96 E-3 ; 7,439 E-3 ; 9,915 E-3 ; 0,015 ; 0,017 ; 0,022 \text { e } 0,025
$$

é necessário estabelecer os valores de $p_{i}$ e $b_{i}, i=1, \cdots, 7$. Conforme já comentamos no Capítulo 3, existem incertezas associadas aos volumes das pipetas e dos balões que 
acarretam incertezas relacionadas à concentração das soluções-padrão.

Segundo o guia EURACHEM as fontes de incerteza que devem ser consideradas para o cálculo de incerteza dos volumes são: a incerteza proveniente da calibração feita pelo fabricante, repetitividade e efeitos de temperatura. Utilizando a lei de propagação de incertezas, apresentada no Capítulo 4 , as incertezas padrão de $p_{0}, b_{0}, p_{i}$ e $b_{i}$ são obtidas e utilizadas para obter a incerteza expandida da concentração $c_{2 i}$.

$\mathrm{Na}$ aplicação consideramos como fontes de incerteza dos volumes, somente a incerteza devida à calibração feita pelo fabricante e a temperatura, pois não se tinha informação de repetitividade. As fórmulas, sugeridas no Guia EURACHEM, para o cálculo das incertezas padrão dos volumes das pipetas e balões são dadas por:

- $u\left(p_{i}\right)=\sqrt{\frac{0,0002 p_{i}}{100}+\frac{4(2,1 E-4) p_{i}}{\sqrt{3}}}$

- $u\left(b_{i}\right)=\sqrt{\frac{U\left(b_{i}\right)}{2}+\frac{4(2,1 E-4) b_{i}}{\sqrt{3}}}$

em que as incertezas padrão devidas à calibração, dos volumes da pipeta $p_{i}$ e do balão $b_{i}$ são $\frac{0,0002 p_{i}}{100}$ e $\frac{U\left(b_{i}\right)}{2}$, respectivamente, sendo $U\left(b_{i}\right)$ a incerteza expandida fornecida pelo fabricante. A expressão $\frac{4(2,1 E-04) V_{i}}{\sqrt{3}}$, em que $V_{i}=p_{i}$ ou $b_{i}$ é a incerteza-padrão devida a efeitos de temperatura asumindo que a distribuição da temperatura é retangular e a faixa de variação da temperatura do reagente utilizado é $\pm 4^{\circ} C$. O coeficiente de expansão do volume da água é $2,1 E-4$.

As informações relativas aos volumes das pipetas e balões inclusive os valores de $p_{0}$ e $b_{0}$ estão apresentadas na Tabela 5.1.

Consideramos como incerteza padrão da concentração de referência $u\left(c_{0}\right)=\frac{U\left(c_{0}\right)}{2}=\frac{3}{2}$, em que a incerteza expandida $U\left(c_{0}\right)=3$ foi declarada pelo fabricante. Consideramos que $u\left(c_{0}\right), u\left(p_{0}\right), u\left(b_{0}\right) u\left(p_{i}\right)$ e $u\left(b_{i}\right), i=1, \cdots, 7$, estão baseadas em $3,10,10,10,10$ graus de liberdade, respectivamente. Fornecemos poucos graus de liberdade para $c_{0}, 3$, porque essa informação é externa (variável do tipo B), para o caso de $p_{0}, b_{0}, p_{i}$, e $b_{i}$ asumimos 10 graus 
Tabela 5.1: Incertezas-padrão dos volumes das pipetas e dos balões

\begin{tabular}{|c|c|c|c|c|}
\hline $\mathrm{i}$ & $p_{i}$ & $u\left(p_{i}\right)$ & $b_{i}$ & $u\left(b_{i}\right)$ \\
\hline 0 & 0,40 & $1,94 \mathrm{E}-04$ & 200,70 & 0,17 \\
1 & 0,25 & $1,21 \mathrm{E}-04$ & 100,35 & 0,09 \\
2 & 0,75 & $3,64 \mathrm{E}-04$ & 200,73 & 0,17 \\
3 & 0,50 & $2,43 \mathrm{E}-04$ & 100,40 & 0,09 \\
4 & 0,75 & $3,64 \mathrm{E}-04$ & 100,19 & 0,09 \\
5 & 0,88 & $4,24 \mathrm{E}-04$ & 100,25 & 0,09 \\
6 & 0,55 & $2,67 \mathrm{E}-04$ & 49,94 & 0,04 \\
7 & 0,63 & $3,03 \mathrm{E}-04$ & 49,89 & 0,04 \\
\hline
\end{tabular}

de liberdade porque no laboratório se fez 11 medições dos volumes, somente com água.

A Tabela 5.2 apresenta as incertezas expandidas relacionadas à concentração das 7 soluções-padrão considerando um nível de confiança de $95 \%$. As incertezas expandidas foram obtidas segundo a solução recomendada pelo ISOGUM, dada em (4.21), e também através da solução em séries, em que $H_{2}=\sum_{i=0}^{2} h_{i}, H_{3}=\sum_{i=0}^{3} h_{i}$ e $H_{4}=\sum_{i=0}^{4} h_{i}$, sendo $h_{i}, i=0, \cdots, 4$ definidas em (4.13), (4.14), (4.15), (4.16) e (4.17), respectivamente.

Observamos na Tabela 5.2 que as incertezas expandidas crescem com o aumento da concentração da solução-padrão. Observamos também, que a incerteza expandida recomendada pelo ISOGUM apresenta os menores valores e que $H_{2}<H_{3}<H_{4}$ para todas as concentrações.

Tabela 5.2: Incertezas expandidas da concentração das soluções-padrão

\begin{tabular}{|c|c|c|c|c|}
\hline $\mathrm{C}_{2 i}$ & ISOGUM & $\mathrm{H}_{2}$ & $\mathrm{H}_{3}$ & $H_{4}$ \\
\hline $4.960 \mathrm{E}-3$ & $2,34 \mathrm{E}-5$ & $2,38 \mathrm{E}-5$ & $2,41 \mathrm{E}-5$ & $2,70 \mathrm{E}-5$ \\
$7.439 \mathrm{E}-3$ & $3,50 \mathrm{E}-5$ & $3,56 \mathrm{E}-5$ & $3,61 \mathrm{E}-5$ & $4,05 \mathrm{E}-5$ \\
$9.915 \mathrm{E}-3$ & $4,67 \mathrm{E}-5$ & $4,75 \mathrm{E}-5$ & $4,82 \mathrm{E}-5$ & $5,39 \mathrm{E}-5$ \\
0.015 & $7,02 \mathrm{E}-5$ & $7,14 \mathrm{E}-5$ & $7,24 \mathrm{E}-5$ & $8,12 \mathrm{E}-5$ \\
0.017 & $8,19 \mathrm{E}-5$ & $8,33 \mathrm{E}-5$ & $8.44 \mathrm{E}-5$ & $9,45 \mathrm{E}-5$ \\
0.022 & $1,03 \mathrm{E}-4$ & $1,05 \mathrm{E}-4$ & $1,07 \mathrm{E}-4$ & $1,19 \mathrm{E}-4$ \\
0.025 & $1,18 \mathrm{E}-4$ & $1,20 \mathrm{E}-4$ & $1,21 \mathrm{E}-4$ & $1,36 \mathrm{E}-4$ \\
\hline
\end{tabular}




\subsection{Aplicação do modelo heteroscedástico}

Nas aplicações apresentadas nesta seção, o interese é estimar a concentração de duas amostras A e B dos elementos químicos cromo, cádmio, chumbo e bário. Utilizamos dados fornecidos pelo IPT, para comparar os resultados obtidos considerando o modelo de calibração heteroscedástico proposto no Capítulo 3 e o modelo usual apresentado no Capítulo 2.

As Tabelas 5.3, 5.6, 5.9 e 5.12 apresentam os valores fixados para as concentrações das soluções-padrão com suas incertezas-padrão e as respectivas intensidades, fornecidas pelo método de espectrometria de plasma. Esses dados correspondem ao primeiro estágio.

As Tabelas 5.4, 5.7, 5.10 e 5.13 apresentam as intensidades correspondentes a 3 soluçõesamostra provenientes das amostras A e B. Esses são os dados do segundo estágio.

Observando as Tabelas 5.3, 5.6, 5.9 e 5.12, verificamos que os valores das incertezaspadrão crescem com o aumento das concentrações. Dessa forma, o modelo de calibração controlada heteroscedástco parece ser mais adequado.

No modelo heteroscedástico proposto no Capítulo 3 , consideramos que as variâncias $\sigma_{\delta_{i}}^{2}$ são conhecidas, portanto assumimos que $\sigma_{\delta_{i}}^{2}=u\left(X_{i}\right)^{2}$, ou seja, $\sigma_{\delta_{i}}^{2}$ é igual ao quadrado da incerteza-padrão correspondente a $i$-ésima solução-padrão.

Como vimos no Capítulo 3, para obter as estimativas dos parâmetros do modelo heteroscedástico, inicialmente temos que estimar $\sigma_{\epsilon}^{2}$ e $\beta$ por meio de um processo iterativo. $\mathrm{O}$ método iterativo utilizado para resolver o sistema de equações (3.27) e (3.28) foi o método de Quasi-Newton (Press, 1992). Utilizamos como ponto inicial para $\beta$ a estimativa obtida do modelo usual e para $\sigma_{\epsilon}^{2}$ a estimativa obtida através da equação (3.15).

As Tabelas 5.5, 5.8, 5.11 e 5.14 apresentam estimativas de $\alpha, \beta, X_{0}, V\left(\hat{X}_{0}\right)$ e a incerteza expandida, $U\left(X_{0}\right)$, sob o modelo heteroscedástico, para as amostras $\mathrm{A}$ e $\mathrm{B}$, do elemento cromo, cádmio, chumbo e bário, respectivamente. São apresentadas, também, as estimativas 
obtidas a partir do modelo usual para compararmos os resultados obtidos sob os dois modelos. As estimativas das variâncias de $\hat{X}_{0}$ foram calculadas segundo (2.10) e (3.29) respectivamente. A estimativa da incerteza expandida, $U\left(X_{0}\right)$, é obtida multiplicando-se a raiz quadrada da estimativa da variância de $\hat{X}_{0}$ por 1,96 .

Pelas Tabelas 5.5, 5.8, 5.11 e 5.14 observamos que as estimativas de $\alpha$ e $\beta$ fornecidas pelo modelo usual são as mesmas para a amostra A e B. Isso ocorre pois sob esse modelo os estimadores de $\alpha$ e $\beta$ só dependem do primeiro estágio. Para o modelo heteroscedástico, vemos que existem diferenças entre as estimativas das duas amostras. Para o chumbo e cádmio as diferenças são muito pequenas e estão próximas das estimativas obtidas usando o modelo usual. Para o bário e cromo parece existir uma diferença maior entre as estimativas das duas amostras e, também, em relação à estimativa obtida sob o modelo usual. Observamos, também, que, para as duas amostras, em geral não existem diferenças entre as estimativas de $X_{0}$ obtidas pelo modelo usual e heteroscedástico para todos os elementos químicos. Notamos que as estimativas da concentração da amostra A do cromo, cádmio e chumbo estão fora dos respectivos intervalos de variação da concentração das soluções-padrão. Verificamos que, com exceção das amostras A e B do chumbo e amostra B do cromo, em geral, as estimativas da variância de $\hat{X}_{0}$ e a incerteza expandida $U\left(X_{0}\right)$ fornecidas pelo modelo usual são maiores. 
Tabela 5.3: Concentração $(m g / g)$, incerteza-padrão e intensidade das soluções-padrão do elemento cromo.

\begin{tabular}{|c|c|c|}
\hline$X_{i}$ & $u\left(X_{i}\right)$ & Intensidade \\
\hline 0,05 & 0,00016 & 6455,900 \\
0,11 & 0,00027 & 13042,933 \\
0,26 & 0,00040 & 32621,733 \\
0,79 & 0,00122 & 97364,500 \\
1,05 & 0,00161 & 129178,100 \\
\hline
\end{tabular}

Tabela 5.4: Intensidade das soluções-amostra das amostras A e B do elemento cromo.

\begin{tabular}{|c|c|}
\hline \multicolumn{2}{|c|}{ Intensidade } \\
\hline Amostra A & Amostra B \\
\hline 1465,0 & 10173,6 \\
1351,0 & 10516,9 \\
1495,6 & 10352,2 \\
\hline
\end{tabular}

Tabela 5.5: Estimativas de $\alpha, \beta, X_{0}, V\left(\hat{X}_{0}\right)$ e $U\left(X_{0}\right)$ segundo o modelo usual e heteroscedástico, para as amostras A e B do elemento cromo.

\begin{tabular}{|c|c|c|c|c|}
\hline \multirow{2}{*}{ Parâmetros } & \multicolumn{2}{|c|}{ Amostra A } & \multicolumn{2}{c|}{ Amostra B } \\
\cline { 2 - 5 } & M-usual & M-heteroscedástico & M-usual & M-heteroscedástico \\
\hline$\alpha$ & 123,574 & 81,566 & 123,574 & 105,979 \\
$\beta$ & 122700 & 122800 & 122700 & 122700 \\
$X_{0}$ & 0,011 & 0,011 & 0,083 & 0,083 \\
$V\left(\hat{X}_{0}\right)$ & $9,80 \mathrm{E}-07$ & $7,47 \mathrm{E}-07$ & $1,16 \mathrm{E}-06$ & $1,05 \mathrm{E}-06$ \\
$\mathrm{U}\left(\mathrm{X}_{0}\right)$ & 0,00194 & 0,00169 & 0,00211 & 0,00201 \\
\hline
\end{tabular}


Tabela 5.6: Concentração $(m g / g)$, incerteza-padrão e intensidade das soluções-padrão do elemento cádmio.

\begin{tabular}{|c|c|c|}
\hline$X_{i}$ & $u\left(X_{i}\right)$ & Intensidade \\
\hline 0,05 & 0,00016 & 4,89733 \\
0,10 & 0,00027 & 9,706 \\
0,25 & 0,00041 & 23,41333 \\
0,73 & 0,00122 & 69,73 \\
1,01 & 0,00168 & 96,85667 \\
\hline
\end{tabular}

Tabela 5.7: Intensidade das soluções-amostra das amostras A e B do elemento cádmio.

\begin{tabular}{|c|c|}
\hline \multicolumn{2}{|c|}{ Intensidade } \\
\hline Amostra A & Amostra B \\
\hline 0,679 & 5,066 \\
0,6837 & 5,027 \\
0,6846 & 5,085 \\
\hline
\end{tabular}

Tabela 5.8: Estimativas de $\alpha, \beta, X_{0}, V\left(\hat{X}_{0}\right)$ e $U\left(X_{0}\right)$ segundo o modelo usual e heteroscedástico, para as amostras $\mathrm{A}$ e $\mathrm{B}$ do elemento cádmio.

\begin{tabular}{|c|c|c|c|c|}
\hline \multirow{2}{*}{ Parâmetros } & \multicolumn{2}{|c|}{ Amostra A } & \multicolumn{2}{c|}{ Amostra B } \\
\cline { 2 - 5 } & M-usual & M-heteroscedástico & M-usual & M-heteroscedástico \\
\hline$\alpha$ & $-0,156$ & $-0,06$ & $-0,156$ & $-0,062$ \\
$\beta$ & 95,828 & 95,602 & 95,828 & 95,608 \\
$X_{0}$ & 0,00875 & 0,00776 & 0,054 & 0,054 \\
$V\left(\hat{X}_{0}\right)$ & $4.055 \mathrm{E}-6$ & $1.538 \mathrm{E}-6$ & $3.808 \mathrm{E}-6$ & $1.479 \mathrm{E}-6$ \\
$\mathrm{U}\left(\mathrm{X}_{0}\right)$ & 0,00395 & 0,00243 & 0,00382 & 0,00238 \\
\hline
\end{tabular}


Tabela 5.9: Concentração $(\mathrm{mg} / \mathrm{g})$, incerteza-padrão e intensidade das soluções-padrão do elemento chumbo.

\begin{tabular}{|c|c|c|}
\hline$X_{i}$ & $u\left(X_{i}\right)$ & Intensidade \\
\hline 0,05 & 0,00015 & 0,9471 \\
0,10 & 0,00025 & 1,46833 \\
0,26 & 0,00039 & 3,09033 \\
0,77 & 0,00117 & 8,40533 \\
1,01 & 0,00155 & 10,92667 \\
\hline
\end{tabular}

Tabela 5.10: Intensidade das soluções-amostra das amostras A e B do elemento chumbo.

\begin{tabular}{|c|c|}
\hline \multicolumn{2}{|c|}{ Intensidade } \\
\hline Amostra A & Amostra B \\
\hline 0,59490 & 1,303 \\
0,58650 & 1,290 \\
0,57270 & 1,341 \\
\hline
\end{tabular}

Tabela 5.11: Estimativas de $\alpha, \beta, X_{0}, V\left(\hat{X}_{0}\right)$ e $U\left(X_{0}\right)$ segundo o modelo usual e heteroscedástico, para as amostras A e B do elemento chumbo.

\begin{tabular}{|c|c|c|c|c|}
\hline \multirow{2}{*}{ Parâmetros } & \multicolumn{2}{|c|}{ Amostra A } & \multicolumn{2}{c|}{ Amostra B } \\
\cline { 2 - 5 } & M-usual & M-heteroscedástico & M-usual & M-heteroscedástico \\
\hline$\alpha$ & 0,441 & 0,445 & 0,441 & 0,442 \\
$\beta$ & 10,352 & 10,343 & 10,352 & 10,349 \\
$X_{0}$ & 0,014 & 0,013 & 0,084 & 0,084 \\
$V\left(\hat{X}_{0}\right)$ & $6,77 \mathrm{E}-07$ & $7,24 \mathrm{E}-07$ & $1,56 \mathrm{E}-06$ & $1,73 \mathrm{E}-06$ \\
$\mathrm{U}\left(\mathrm{X}_{0}\right)$ & 0,00161 & 0,00167 & 0,00245 & 0,00258 \\
\hline
\end{tabular}


Tabela 5.12: Concentração $(\mathrm{mg} / \mathrm{g})$, incerteza-padrão e intensidade das soluçõespadrão do elemento bário.

\begin{tabular}{|c|c|c|}
\hline$X_{i}$ & $u\left(X_{i}\right)$ & Intensidade \\
\hline 0,10 & 0,00026 & 184857,167 \\
0,21 & 0,00054 & 372903,800 \\
0,51 & 0,00127 & 912606,300 \\
1,06 & 0,00266 & 1923938,100 \\
1,60 & 0,00400 & 2913721,933 \\
\hline
\end{tabular}

Tabela 5.13: Intensidade das soluções-amostra das amostras A e B do elemento bário.

\begin{tabular}{|c|c|}
\hline \multicolumn{2}{|c|}{ Intensidade } \\
\hline Amostra A & Amostra B \\
\hline 279034,2 & 2640425,3 \\
279562,1 & 2661015,0 \\
278462,2 & 2639452,0 \\
\hline
\end{tabular}

Tabela 5.14: Estimativas de $\alpha, \beta, X_{0}, V\left(\hat{X}_{0}\right)$ e $U\left(X_{0}\right)$ segundo o modelo usual e heteroscedástico, para as amostras A e B do elemento bário.

\begin{tabular}{|c|c|c|c|c|}
\hline \multirow{2}{*}{ Parâmtros } & \multicolumn{2}{|c|}{ Amostra A } & \multicolumn{2}{c|}{ Amostra B } \\
\cline { 2 - 5 } & M-usual & M-heteroscedástico & M-usual & M-heteroscedástico \\
\hline$\alpha$ & -11670 & -19240 & -11670 & -10340 \\
$\beta$ & 1822000 & 1832000 & 1822000 & 1820000 \\
$\hat{X}_{0}$ & 0,16 & 0,163 & 1,46 & 1,46 \\
$V\left(\hat{X}_{0}\right)$ & $3,09 \mathrm{E}-06$ & $7,15 \mathrm{E}-07$ & $1,39 \mathrm{E}-05$ & $2,19 \mathrm{E}-05$ \\
$\mathrm{U}\left(\mathrm{X}_{0}\right)$ & 0,00344 & 0,00166 & 0,00731 & 0,00917 \\
\hline
\end{tabular}




\section{CAPÍtulo 6}

\section{Comentários finais}

O estudo dos processos químicos, realizados com o objetivo de determinar a concentração de um elemento químico em uma amostra, mostrou a necessidade de desenvolvermos modelos de calibração que incorporassem os erros provenientes da preparação das soluções-padrão. Assim, neste trabalho, inicialmente, propomos modelos de calibração homoscedástico. Como observamos que as incertezas associadas às soluções-padrão dependem dos valores das concentrações consideradas, propomos também um modelo de calibração controlada heteroscedástico, que parece ser mais apropriado para descrever os processos.

Verificamos que, apesar das estimativas da concentração da solução-amostra, obtidas sob os modelos propostos e o modelo usual recomendado pelo guia EURACHEM, serem, em geral, muito próximas, as respectivas estimativas das variâncias e conseqüentemente as incertezas expandidas podem diferir. Portanto, embora o uso do modelo usual venha sendo justificado considerando que os erros das soluções-padrão sejam desprezíveis, nem sempre esse modelo parece ser adequado.

Dando continuidade aos estudos realizados nesse trabalho, seria interessante considerar modelos de calibração controlada que incorporem, também, erros provenientes da preparação da solução-amostra.

Estender os modelos de calibração controlada considerando heteroscedasticidade dos 
erros $\epsilon$, ou adotando modelos não lineares, ou, ainda, assumindo distribuição assimétrica para os erros, poderiam ser também objetos de pesquisas futuros.

Além desses tópicos, o problema poderia ser explorado utilizando-se modelos Bayessianos. 


\section{Apêndice 1.}

Teorema 1. No modelo homoscedástico, os estimadores de mínimos quadrados de $\alpha, \beta$ e $X_{0}$ são estimadores lineares não viciados de variância mínima.

\section{Prova:}

A demonstração será feita apenas para o estimador $\hat{\alpha}$, pois as demonstrações para $\hat{\beta}$ e $\hat{X}_{0}$ são análogas.

Como estamos restringindo à classe dos estimadores lineares, temos que $\hat{\alpha}=\sum_{j=1}^{n} a_{j} Y_{j}+$ $\sum_{j=n+1}^{n+k} a_{j} Y_{0 j}$. Devemos encontrar as constantes $a_{j}, j=1, \cdots, n+k$, tais que:

(a) $E(\hat{\alpha})=\alpha$, ou seja $\hat{\alpha}$ é um estimador não viciado de $\alpha$.

(b) $V(\hat{\alpha})$ é mínima quando comparada à variância de todos os estimadores lineares que satisfazem (a).

De (a) temos que,

$$
\begin{aligned}
\alpha=E(\hat{\alpha}) & =\sum_{j=1}^{n} a_{j} E\left(Y_{j}\right)+\sum_{j=n+1}^{n+k} a_{j} E\left(Y_{0 j}\right) \\
& =\sum_{j=1}^{n} a_{j}\left(\alpha+\beta X_{j}\right)+\sum_{j=n+1}^{n+k} a_{j}\left(\alpha+\beta X_{0}\right) \\
& =\alpha \sum_{j=1}^{n+k} a_{j}+\beta\left(\sum_{j=1}^{n} a_{j} X_{j}+\sum_{j=n+1}^{n+k} a_{j} X_{0}\right)
\end{aligned}
$$

logo, temos duas equações que devem estar satisfeitas

$$
\sum_{j=1}^{n+k} a_{j}=1 \text { e } \sum_{j=1}^{n} a_{j} X_{j}+\sum_{j=n+1}^{n+k} a_{j} X_{0}=0 .
$$

Equivalentemente temos

$$
\sum_{j=1}^{n} a_{j}=1, \quad \sum_{j=n+1}^{n+k} a_{j}=0 \quad \text { e } \sum_{j=1}^{n} a_{j} X_{j}=0 .
$$


De (b) temos que

$$
\begin{aligned}
V(\hat{\alpha}) & =E\left[(\hat{\alpha}-\alpha)^{2}\right]=E\left[\left(\sum_{j=1}^{n} a_{j} Y_{j}+\sum_{j=n+1}^{n+k} a_{j} Y_{0 j}-\alpha\right)^{2}\right] \\
& =E\left[\left(\sum_{j=1}^{n} a_{j}\left(\alpha+\beta X_{j}+\epsilon_{j}-\beta \delta_{j}\right)+\sum_{j=n+1}^{n+k} a_{j}\left(\alpha+\beta X_{0}+\epsilon_{j}\right)-\alpha\right)^{2}\right] .
\end{aligned}
$$

Pelas restrições dadas em (1) temos que

$$
\begin{aligned}
V(\hat{\alpha}) & =E\left[\left(\sum_{j=1}^{n} a_{j}\left(\epsilon_{j}-\beta \delta_{j}\right)+\sum_{j=n+1}^{n+k} a_{j} \epsilon_{j}\right)^{2}\right] \\
& =E\left[\left(\sum_{j=1}^{n} a_{j}\left(\epsilon_{j}-\beta \delta_{j}\right)\right)^{2}\right]+E\left[\left(\sum_{j=n+1}^{n+k} a_{j} \epsilon_{j}\right)^{2}\right] \\
& =\left(\beta^{2} \sigma_{\delta}^{2}+\sigma_{\epsilon}^{2}\right) \sum_{j=1}^{n} a_{j}^{2}+\sigma_{\epsilon}^{2} \sum_{j=n+1}^{n+k} a_{j}^{2} .
\end{aligned}
$$

Para que $V(\hat{\alpha})$ seja mínima devemos minimizar $\sum_{j=1}^{n+k} a_{j}^{2}$. Temos, então, que encontrar constantes $a_{j}$ tais que minimizem $\sum_{j=1}^{n+k} a_{j}^{2}$ sobre as restrições dadas em (1). Utilizando a teoria de multiplicadores de Lagrange, temos que minimizar

$$
L=\sum_{j=1}^{n+k} a_{j}^{2}-\lambda_{1}\left(\sum_{j=1}^{n} a_{j}-1\right)-\lambda_{2}\left(\sum_{j=1}^{n} a_{j} X_{j}\right)-\lambda_{3}\left(\sum_{j=n+1}^{n+k} a_{j}\right) .
$$

Derivando a expressão (2) temos as seguintes equações

$$
\begin{aligned}
& \frac{\partial L}{\partial a_{j}}=2 a_{j}-\lambda_{1}-\lambda_{2} X_{j}=0, \quad j=1,2, \cdots, n \\
& \frac{\partial L}{\partial a_{j}}=2 a_{j}-\lambda_{3}=0, \quad j=n+1, n+2, \cdots, n+k \\
& \frac{\partial L}{\partial \lambda_{1}}=-\sum_{j=1}^{n} a_{j}+1=0 \\
& \frac{\partial L}{\partial \lambda_{2}}=-\sum_{j=1}^{n} a_{j} X_{j}=0 \\
& \frac{\partial L}{\partial \lambda_{3}}=-\sum_{j=n+1}^{n+k} a_{j}=0 .
\end{aligned}
$$

Somando as $n$ primeiras equações e considerando a equação (1), temos

$$
2=n \lambda_{1}+\lambda_{2} \sum_{j=1}^{n} X_{j}
$$


Multiplicando a j-ésima equação em (3) por $X_{j}$ e somando as $n$ equações, temos

$$
2 \sum_{j=1}^{n} X_{j} a_{j}=\lambda_{1} \sum_{j=1}^{n} X_{j}+\lambda_{2} \sum_{j=1}^{n} X_{j}^{2} .
$$

Como $\sum_{j=1}^{n} a_{j} X_{j}=0$, temos

$$
\lambda_{1}=-\lambda_{2} \frac{\sum_{j=1}^{n} X_{j}^{2}}{\sum_{j=1}^{n} X_{j}} .
$$

Substituindo esta última expressão em (8), temos

$$
\lambda_{2}=\frac{-2 \sum_{j=1}^{n} X_{j} / n}{\sum_{j=1}^{n} X_{j}^{2}-n \bar{X}^{2}}=\frac{-2 \bar{X}}{\sum_{j=1}^{n}\left(X_{j}-\bar{X}\right)^{2}}
$$

e

$$
\lambda_{1}=\frac{2 \sum_{j=1}^{n} X_{j}^{2} / n}{\sum_{j=1}^{n}\left(X_{j}-\bar{X}\right)^{2}} .
$$

Substituindo $\lambda_{1}$ e $\lambda_{2}$ na $j$-ésima equação em (1) e isolando $a_{j}$ temos

$$
a_{j}=\frac{\sum_{j=1}^{n} X_{j}^{2} / n-\bar{X} X_{t}}{\sum_{j=1}^{n}\left(X_{j}-\bar{X}\right)^{2}} \text { para } j=1, \cdots, n .
$$

Somando as $k$ equações de (4) e utilizando (7) temos que $\lambda_{3}=0$, logo por (4) temos que $a_{j}=0$ para $j=n+1, \cdots, n+k$.

Portanto, o melhor estimador linear não viciado de $\alpha$ é

$$
\hat{\alpha}=\sum_{j=1}^{n} a_{j} Y_{j}=\frac{\bar{Y} \sum_{j=1}^{n} X_{j}^{2}-\bar{X} \sum_{j=1}^{n} Y_{j} X_{j}}{\sum_{j=1}^{n}\left(X_{j}-\bar{X}\right)^{2}}=\bar{Y}-\hat{\beta} \bar{X},
$$

ou seja, é o estimador de mínimos quadrados. 


\section{Apêndice 2.}

\section{Incerteza expandida obtida por meio da solução em séries}

Seja $Z$ uma variável aleatória com distribuição normal com média $\eta$ e variância $\sigma_{Z}^{2}=$ $\sum_{i=1}^{m} \lambda_{i} \sigma_{i}^{2}$, em que $\lambda_{i}$ e $\sigma_{i}^{2}, i=1, \cdots, m$, são, respectivamente, constantes conhecidas e variâncias desconhecidas. Para obter um intervalo de confiança para o parâmetro $\eta$, é necessário encontrar uma função $h\left(S_{1}^{2}, S_{2}^{2}, \cdots, S_{m}^{2}\right)$, sendo $S_{i}^{2}$ um estimador não viesado de $\sigma_{i}^{2}$, tal que, para um nível de confiança $p$ se tenha

$$
P\left[(Z-\eta) \leq h\left(S_{1}^{2}, S_{2}^{2}, \cdots, S_{m}^{2}, p\right)\right]=p .
$$

Apresentamos um esboço da prova da solução em séries dado em Welch (1947 (a) e 1947 (b)).

Seja a probabilidades condicional

$$
\begin{aligned}
\Phi\left(\frac{h\left(S_{1}^{2}, S_{2}^{2}, \cdots, S_{m}^{2}, p\right)}{\sqrt{\sum_{i=1}^{m} \lambda_{i} \sigma_{i}^{2}}}\right) & =P\left[(Z-\eta) \leq h\left(S_{1}^{2}, S_{2}^{2}, \cdots, S_{m}^{2}, p\right) /\left(S_{1}^{2}, S_{2}^{2}, \cdots, S_{m}^{2}\right)\right] \\
& =\int_{-\infty}^{\frac{h\left(S_{1}^{2}, S_{2}^{2}, \cdots, S_{m}^{2}, p\right)}{\sqrt{\sum_{i=1}^{m} \lambda_{i} \sigma_{i}^{2}}}} \frac{1}{\sqrt{2 \pi}} e^{-\frac{1}{2} \mu^{2}} d \mu
\end{aligned}
$$

Para facilitar vamos denotar $\Phi\left(\frac{h\left(S_{1}^{2}, S_{2}^{2}, \cdots, S_{m}^{2}, p\right)}{\sqrt{\sum_{i=1}^{m} \lambda_{i} \sigma_{i}^{2}}}\right)=\Phi\left(h\left(S_{1}^{2}, S_{2}^{2}, \cdots, S_{m}^{2}, p\right)\right)$. De (10) temos que

$P\left[(Z-\eta) \leq h\left(S_{1}^{2}, S_{2}^{2}, \cdots, S_{m}^{2}, p\right)\right]=\int_{S_{1}^{2}} \cdots \int_{S_{m}^{2}} \Phi\left(h\left(S_{1}^{2}, S_{2}^{2}, \cdots, S_{m}^{2}, p\right)\right) \prod_{i=1}^{m} g\left(S_{i}^{2}\right) d S_{1}^{2}, \cdots, d S_{m}^{2}$,

em que $g\left(S_{i}^{2}\right)$ é função de densidade de $S_{i}^{2}$. Expandindo $\Phi\left(h\left(S_{1}^{2}, S_{2}^{2}, \cdots, S_{m}^{2}, p\right)\right)$ em série de Taylor em torno de $\left(\sigma_{1}^{2}, \cdots, \sigma_{m}^{2}\right)$ temos que

$$
\begin{aligned}
\Phi\left(h\left(S_{1}^{2}, S_{2}^{2}, \cdots, S_{m}^{2}, p\right)\right) & =\Phi\left(h\left(\sigma_{1}^{2}, \sigma_{2}^{2}, \cdots, \sigma_{m}^{2}, p\right)\right) \\
& +D \Phi\left(h\left(S_{1}^{2}, S_{2}^{2}, \cdots, S_{m}^{2}, p\right)\right)\left[\left(S_{1}^{2}, S_{2}^{2}, \cdots, S_{m}^{2}\right)-\left(\sigma_{1}^{2}, \sigma_{2}^{2}, \cdots, \sigma_{m}^{2}\right)\right] \\
& +\frac{1}{2 !} D^{2} \Phi\left(h\left(S_{1}^{2}, S_{2}^{2}, \cdots, S_{m}^{2}, p\right)\right)^{2}\left[\left(S_{1}^{2}, S_{2}^{2}, \cdots, S_{m}^{2}\right)-\left(\sigma_{1}^{2}, \sigma_{2}^{2}, \cdots, \sigma_{m}^{2}\right)\right]^{2} \\
& +\cdots
\end{aligned}
$$


$\mathrm{ou}$

$$
\begin{aligned}
\Phi\left(h\left(S_{1}^{2}, S_{2}^{2}, \cdots, S_{m}^{2}, p\right)\right) & =\left[1+\left\{\left(S_{1}^{2}, S_{2}^{2}, \cdots, S_{m}^{2}\right)-\left(\sigma_{1}^{2}, \sigma_{2}^{2}, \cdots, \sigma_{m}^{2}\right)\right\} D\right. \\
& \left.+\left\{\left(S_{1}^{2}, S_{2}^{2}, \cdots, S_{m}^{2}\right)-\left(\sigma_{1}^{2}, \sigma_{2}^{2}, \cdots, \sigma_{m}^{2}\right)^{2}\right\} D^{2}+\cdots\right] \Phi\left(h \left(\sigma_{1}^{2}, \sigma_{2}^{2}, \cdots, \sigma_{m}^{2}, p\right.\right.
\end{aligned}
$$

em que $D=\left(\partial_{1}, \cdots, \partial_{m}\right)$ e $\partial_{i} \Phi\left(h\left(S_{1}^{2}, S_{2}^{2}, \cdots, S_{m}^{2}, p\right)=\left.\frac{\partial}{\partial S_{i}^{2}} \Phi\left(h\left(S_{1}^{2}, S_{2}^{2}, \cdots, S_{m}^{2}, p\right)\right)\right|_{S_{i}^{2}=\sigma_{i}^{2}, i=1, \cdots, m}\right.$.

A igualdade anterior é reescrita como

$$
\Phi\left(h\left(S_{1}^{2}, S_{2}^{2}, \cdots, S_{m}^{2}, p\right)\right)=\exp \left[\sum_{i=1}^{m}\left(S_{i}^{2}-\sigma_{i}^{2}\right) \partial_{i}\right] \Phi\left(h\left(S_{1}^{2}, S_{2}^{2}, \cdots, S_{m}^{2}, p\right)\right) .
$$

Substituindo a equação (12) em (11) temos que

$$
\begin{aligned}
& P\left[(Z-\eta) \leq h\left(S_{1}^{2}, S_{2}^{2}, \cdots, S_{m}^{2}, p\right)\right]= \\
& \quad \int_{S_{1}^{2}} \cdots \int_{S_{m}^{2}} \exp \left[\sum_{i=1}^{m}\left(S_{i}^{2}-\sigma_{i}^{2}\right) \partial_{i}\right] \Phi\left(h\left(S_{1}^{2}, S_{2}^{2}, \cdots, S_{m}^{2}, p\right)\right) \prod_{i=1}^{m} g\left(S_{i}^{2}\right) d S_{1}^{2}, \cdots, d S_{m}^{2} \\
& \quad=\left[\prod_{i=1}^{m} \int_{S_{i}^{2}} \exp \left[\sum_{i=1}^{m}\left(S_{i}^{2}-\sigma_{i}^{2}\right) \partial_{i}\right] g\left(S_{i}^{2}\right) d S_{i}^{2}\right] \Phi\left(h\left(S_{1}^{2}, S_{2}^{2}, \cdots, S_{m}^{2}, p\right)\right) \\
& \quad=\Theta \Phi\left(h\left(S_{1}^{2}, S_{2}^{2}, \cdots, S_{m}^{2}, p\right)\right) .
\end{aligned}
$$

A equação (13) é conhecida como a solução condensada, em que

$$
\Theta=\left[\prod_{i=1}^{m} \int_{S_{i}^{2}} \exp \left[\sum_{i=1}^{m}\left(S_{i}^{2}-\sigma_{i}^{2}\right) \partial_{i}\right] g\left(S_{i}^{2}\right) d S_{i}^{2}\right] .
$$

A função de densidade de $S_{i}^{2}$ (Student, 1908) é aproximada pela curva de tipo III de Pearson dada por

$$
p\left(S_{i}^{2}\right) d S_{i}^{2}=\frac{1}{\Gamma\left(\frac{1}{2} f_{i}\right)}\left(\frac{f_{i} S_{i}^{2}}{2 \sigma_{i}^{2}}\right)^{\frac{1}{2} f_{i}-1} \exp \left(-\frac{1}{2} \frac{f_{i} S_{i}^{2}}{2 \sigma_{i}^{2}}\right) d\left(\frac{f_{i} S_{i}^{2}}{2 \sigma_{i}^{2}}\right)
$$


Substituindo (15) e $S_{i}^{2}=\frac{2 \sigma_{i}^{2} \mu}{f_{i}}$ em (14) temos que

$$
\begin{aligned}
\Theta & =\prod_{i=1}^{m} \int_{S_{i}^{2}} \exp \left(-\sigma_{1}^{2} \partial_{i}\right) \exp \left(\left(\partial_{i}-\frac{f_{i}}{2 \sigma_{i}^{2}}\right) \frac{2}{f_{i}} \sigma_{i}^{2} \mu\right) \frac{1}{\Gamma\left(\frac{1}{2} f_{i}\right)} \mu^{\frac{1}{2} f_{i}-1} d \mu \\
& =\prod_{i=1}^{m} \frac{\exp \left(-\sigma_{1}^{2} \partial_{i}\right)}{\left[\left(\frac{f_{i}}{2 \sigma_{i}^{2}}-\partial_{i}\right) \frac{2 \sigma_{i}^{2}}{f_{i}}\right]^{\frac{1}{2} f_{i}}} \\
& =\exp \left(-\sum_{i=1}^{m} \sigma_{i}^{2} \partial_{i}-\frac{1}{2} \sum_{i=1}^{m} \log \left(1-\frac{2}{f_{i}} \sigma_{i}^{2} \partial_{i}\right)\right) \\
& =\exp \left(\sum_{i=1}^{m} \frac{\sigma_{i}^{4} \partial_{i}^{2}}{f_{i}}+\frac{4}{3} \sum_{i=1}^{m} \frac{\sigma_{i}^{6} \partial_{i}^{3}}{f_{i}^{2}}+2 \sum_{i=1}^{m} \frac{\sigma_{i}^{8} \partial_{i}^{4}}{f_{i}^{3}}+\cdots\right) \\
& =1+\sum_{i=1}^{m} \frac{\sigma_{i}^{4} \partial_{i}^{2}}{f_{i}}+\left(\frac{4}{3} \sum_{i=1}^{m} \frac{\sigma_{i}^{6} \partial_{i}^{3}}{f_{i}^{2}}+\frac{1}{2}\left(\sum_{i=1}^{m} \frac{\sigma_{i}^{4} \partial_{i}^{2}}{f_{i}}\right)^{2}\right)+\cdots
\end{aligned}
$$


Por outro lado, para um dado nível de confiança $p$ e para $S_{i}^{2}=s_{i}^{2}$ temos que

$$
p=P\left[\frac{Z-\eta}{\sqrt{\sum_{i=1}^{m} \lambda_{i} \sigma_{i}^{2}}} \leq \xi\right]=\Phi(\xi)
$$

em que $\xi=\frac{h\left(s_{1}^{2}, s_{2}^{2}, \cdots, s_{m}^{2}, p\right)}{\sqrt{\sum_{i=1}^{m} \lambda_{i} \sigma_{i}^{2}}}$. Expandindo a função $\Phi\left(\frac{h\left(S_{1}^{2}, S_{2}^{2}, \cdots, S_{m}^{2}, p\right)}{\sqrt{\sum_{i=1}^{m} \lambda_{i} \sigma_{i}^{2}}}\right)$ em série de Taylor em torno de $\xi$ temos que

$$
\Phi\left(\frac{h\left(S_{1}^{2}, S_{2}^{2}, \cdots, S_{m}^{2}, p\right)}{\sqrt{\sum_{i=1}^{m} \lambda_{i} \sigma_{i}^{2}}}\right)=\exp \left[\left(\frac{h\left(S_{1}^{2}, S_{2}^{2}, \cdots, S_{m}^{2}, p\right)}{\sqrt{\sum_{i=1}^{m} \lambda_{i} \sigma_{i}^{2}}}-\xi\right) \frac{d}{d v}\right] \Phi(v)
$$

Substituindo (17) na equação (13) temos que

$$
\Theta \exp \left[\left(\frac{h\left(S_{1}^{2}, S_{2}^{2}, \cdots, S_{m}^{2}, p\right)}{\sqrt{\sum_{i=1}^{m} \lambda_{i} \sigma_{i}^{2}}}-\xi\right) \frac{d}{d v}\right] \Phi(v)=\Phi(\xi) .
$$

Para resolver a equação (18) em $h\left(S_{1}^{2}, S_{2}^{2}, \cdots, S_{m}^{2}, p\right)$ Welch considera

$$
\begin{aligned}
h\left(S_{1}^{2}, S_{2}^{2}, \cdots, S_{m}^{2}, p\right) & =h_{0}\left(S_{1}^{2}, S_{2}^{2}, \cdots, S_{m}^{2}, p\right)+h_{1}\left(S_{1}^{2}, S_{2}^{2}, \cdots, S_{m}^{2}, p\right) \\
& +h_{2}\left(S_{1}^{2}, S_{2}^{2}, \cdots, S_{m}^{2}, p\right)+\cdots
\end{aligned}
$$

em que

$$
h_{0}\left(S_{1}^{2}, S_{2}^{2}, \cdots, S_{m}^{2}, p\right)=\xi \sqrt{\sum_{i=1}^{m} \lambda_{i} S_{i}^{2}},
$$

e $h_{1}\left(S_{1}^{2}, S_{2}^{2}, \cdots, S_{m}^{2}, p\right)$ incluem termos de ordem $\frac{1}{f_{i}}, h_{2}\left(S_{1}^{2}, S_{2}^{2}, \cdots, S_{m}^{2}, p\right)$ de ordem $\frac{1}{f_{i}^{2}}$, e assim por diante. Desprezando os termos de ordem $\frac{1}{f_{i}^{3}}$, temos que

$$
\begin{aligned}
h\left(S_{1}^{2}, S_{2}^{2}, \cdots, S_{m}^{2}, p\right) & =h_{0}\left(S_{1}^{2}, S_{2}^{2}, \cdots, S_{m}^{2}, p\right)+h_{1}\left(S_{1}^{2}, S_{2}^{2}, \cdots, S_{m}^{2}, p\right) \\
& +h_{2}\left(S_{1}^{2}, S_{2}^{2}, \cdots, S_{m}^{2}, p\right)
\end{aligned}
$$

Logo,

$$
\begin{gathered}
{\left[\frac{h\left(S_{1}^{2}, S_{2}^{2}, \cdots, S_{m}^{2}, p\right)}{\sqrt{\sum_{i=1}^{m} \lambda_{i} \sigma_{i}^{2}}}-\xi\right] \frac{d}{d v}=\left[\frac{h_{0}\left(S_{1}^{2}, S_{2}^{2}, \cdots, S_{m}^{2}, p\right)}{\sqrt{\sum_{i=1}^{m} \lambda_{i} \sigma_{i}^{2}}}-\xi\right] \frac{d}{d v}} \\
+\left[\frac{h_{1}\left(S_{1}^{2}, S_{2}^{2}, \cdots, S_{m}^{2}, p\right)+h_{2}\left(S_{1}^{2}, S_{2}^{2}, \cdots, S_{m}^{2}, p\right)}{\sqrt{\sum_{i=1}^{m} \lambda_{i} \sigma_{i}^{2}}}\right] \frac{d}{d v}
\end{gathered}
$$


Substituindo a equação (21) na equação (18) temos

$$
\begin{aligned}
\Theta \exp & {\left[\left(\frac{h_{0}\left(S_{1}^{2}, S_{2}^{2}, \cdots, S_{m}^{2}, p\right)}{\sqrt{\sum_{i=1}^{m} \lambda_{i} \sigma_{i}^{2}}}-\xi\right) \frac{d}{d v}\right.} \\
+ & \left.\left(\frac{h_{1}\left(S_{1}^{2}, S_{2}^{2}, \cdots, S_{m}^{2}, p\right)+h_{2}\left(S_{1}^{2}, S_{2}^{2}, \cdots, S_{m}^{2}, p\right)}{\sqrt{\sum_{i=1}^{m} \lambda_{i} \sigma_{i}^{2}}}\right) \frac{d}{d v}\right] \Phi(v)=\Phi(\xi) .
\end{aligned}
$$

Por simplicidade denotaremos $h_{i}\left(S_{1}^{2}, S_{2}^{2}, \cdots, S_{m}^{2}, p\right)=h_{i}(\mathbf{V}), i=0,1,2$, em que $\mathbf{V}=$ $\left(S_{1}^{2}, S_{2}^{2}, \cdots, S_{m}^{2}, p\right)$. Da expressão (22) temos

$$
\begin{array}{r}
\Theta \exp \left[\left(\sqrt{\frac{\sum_{i=1}^{m} \lambda_{i} S_{i}^{2}}{\sum_{i=1}^{m} \lambda_{i} \sigma_{i}^{2}}}-1\right) \xi \frac{d}{d v}\right]\left[1+\frac{h_{1}(\mathbf{V})}{\sqrt{\sum_{i=1}^{m} \lambda_{i} \sigma_{i}^{2}}} \frac{d}{d v}\right. \\
\left.+\left(\frac{h_{2}(\mathbf{V})}{\sqrt{\sum_{i=1}^{m} \lambda_{i} \sigma_{i}^{2}}} \frac{d}{d v}+\frac{h_{1}^{2}(\mathbf{V})}{2 \sum_{i=1}^{m} \lambda_{i} \sigma_{i}^{2}} \frac{d^{2}}{d v^{2}}\right)+\cdots\right] \Phi(v)=\Phi(\xi) .
\end{array}
$$

Substituindo a expressão (16) na equação (23) temos

$$
\begin{aligned}
& {\left[\frac{h_{1}(\mathbf{V})}{\sqrt{\sum_{i=1}^{m} \lambda_{i} \sigma_{i}^{2}}} \frac{d}{d v}+\frac{\sum_{i=1}^{m} \sigma_{i}^{4} \partial_{i}^{2}}{f_{i}} \exp \left(\left(\sqrt{\frac{\sum_{i=1}^{m} \lambda_{i} S_{i}^{2}}{\sum_{i=1}^{m} \lambda_{i} \sigma_{i}^{2}}}-1\right) \xi \frac{d}{d v}\right)\right] \Phi(v)+\left[\frac{h_{2}(\mathbf{V})}{\sqrt{\sum_{i=1}^{m} \lambda_{i} \sigma_{i}^{2}}} \frac{d}{d v}\right.} \\
& \quad+\frac{h_{1}^{2}(\mathbf{V})}{2 \sum_{i=1}^{m} \lambda_{i} \sigma_{i}^{2}} \frac{d^{2}}{d v^{2}}+\frac{\sum_{i=1}^{m} \sigma_{i}^{4} \partial_{i}^{2}}{f_{i}} \exp \left(\left(\sqrt{\frac{\sum_{i=1}^{m} \lambda_{i} S_{i}^{2}}{\sum_{i=1}^{m} \lambda_{i} \sigma_{i}^{2}}}-1\right) \xi \frac{d}{d v}\right) \frac{h_{1}(\mathbf{V})}{\sqrt{\sum_{i=1}^{m} \lambda_{i} \sigma_{i}^{2}}} \\
& \left.+\left(\frac{4}{3} \frac{\sum_{i=1}^{m} \sigma_{i}^{6} \partial_{i}^{3}}{f_{i}^{2}}+\frac{1}{2}\left(\frac{\sum_{i=1}^{m} \sigma_{i}^{4} \partial_{i}^{2}}{f_{i}}\right)^{2}\right) \exp \left(\left(\sqrt{\frac{\sum_{i=1}^{m} \lambda_{i} S_{i}^{2}}{\sum_{i=1}^{m} \lambda_{i} \sigma_{i}^{2}}}-1\right) \xi \frac{d}{d v}\right)\right] \Phi(v)=0 .
\end{aligned}
$$

A partir do termo de primeira ordem dessa equação temos

$$
h_{1}(\mathbf{V})=\frac{\xi\left(1+\xi^{2}\right)}{4} \frac{\sum_{i=1}^{m} \frac{\lambda_{i}^{2} S_{i}^{4}}{f_{i}}}{\left(\sum_{i=1}^{m} \lambda_{i} S_{i}^{2}\right)^{\frac{3}{2}}},
$$

de forma análoga obtém-se $h_{2}(\mathbf{V})$, assim a equação (20) é dada por:

$$
\begin{aligned}
h(\mathbf{V}) & =\xi \sqrt{\sum_{i=1}^{m} \lambda_{i} S_{i}^{2}}\left[1+\frac{\left(1+\xi^{2}\right)}{4} \frac{\sum_{i=1}^{m} \frac{\lambda_{i}^{2} S_{i}^{4}}{f_{i}}}{\left(\sum_{i=1}^{m} \lambda_{i} S_{i}^{2}\right)^{2}}-\left(1+\xi^{2}\right) \frac{\sum_{i=1}^{m} \frac{\lambda_{i}^{2} S_{i}^{4}}{f_{i}}}{\left(\sum_{i=1}^{m} \lambda_{i} S_{i}^{2}\right)^{2}}\right. \\
& \left.+\frac{\left(3+5 \xi^{2}+\xi^{4}\right)}{3} \frac{\sum_{i=1}^{m} \frac{\lambda_{i}^{3} S_{i}^{6}}{f_{i}^{2}}}{\left(\sum_{i=1}^{m} \lambda_{i} S_{i}^{2}\right)^{3}}-\frac{\left(15+32 \xi^{2}+9 \xi^{4}\right)}{32} \frac{\left(\sum_{i=1}^{m} \frac{\lambda_{i}^{2} S_{i}^{4}}{f_{i}}\right)^{2}}{\left(\sum_{i=1}^{m} \lambda_{i} S_{i}^{2}\right)^{4}}\right]
\end{aligned}
$$

Particularmente, quando $m=1$ temos o resultado obtido por Fisher (1941), ou seja,

$$
t_{p}=\xi\left[1+\frac{1+\xi^{2}}{4 f}+\frac{3+16 \xi^{2}+5 \xi^{4}}{96 f^{2}}+\cdots\right]
$$

Aspin (1948) desprezou apenas os termos de ordem $\frac{1}{f_{i}^{5}}$, obtendo $h_{3}$ e $h_{4}$ na expressão (19). 


\section{Apêndice 3.}

\section{Incerteza expandida recomendada pelo ISOGUM}

A seguir apresentamos o resultado dado em Welch (1947 (a)) que é aplicado para calcular a incerteza expandida.

Resultado: Seja a variável aleatória $Z \sim N\left(\eta, \sum_{i=1}^{m} \lambda_{i} \sigma_{i}^{2}\right)$, em que $\lambda_{i}, i=1, \cdots, m$ são constantes conhecidas e $\sigma_{i}^{2}, i=1, \cdots, m$ são variâncias desconhecidas. Seja $S_{i}^{2}$, baseado em $f_{i}$ graus de liberdade, um estimador não viesado da variância populacional $\sigma_{i}^{2}$, então a variável

$$
\frac{Z-\eta}{\sqrt{\sum_{i=1}^{m} \lambda_{i} S_{i}^{2}}}
$$

segue uma distribuição aproximadamente t-Student com $f$ graus de liberdade.

Prova: apresentamos a prova apenas para $m=2$.

Seja $V=\frac{Z-\eta}{\sqrt{\lambda_{1} S_{1}^{2}+\lambda_{2} S_{2}^{2}}}$ e $Y=\frac{Z-\eta}{\sqrt{\lambda_{1} \sigma_{1}^{2}+\lambda_{2} \sigma_{2}^{2}}}$, em que $Y^{2}$ tem distribuição qui-quadrado com um grau de liberdade. Dessas dois igualdades temos que

$$
V=\frac{Y}{\sqrt{a_{1} \chi_{1}^{2}+a_{2} \chi_{2}^{2}}}=\frac{Y}{\sqrt{S}}
$$

em que $S=a_{1} \chi_{1}^{2}+a_{2} \chi_{2}^{2}, a_{1}=\frac{\frac{\lambda_{1} \sigma_{1}^{2}}{f_{1}}}{\sum_{i=1}^{2} \lambda_{i} \sigma_{i}^{2}}, a_{2}=\frac{\frac{\lambda_{2} \sigma_{2}^{2}}{f_{2}}}{\sum_{i=1}^{2} \lambda_{i} \sigma_{i}^{2}}, \chi_{i}^{2}=\frac{f_{i}}{\sigma_{i}^{2}} S_{i}^{2}, i=1,2 . \chi_{1}^{2}$ e $\chi_{2}^{2}$ tem distribuição qui-quadrado com $f_{1}$ e $f_{2}$ graus de liberdade, respectivamente. Quando $a_{1}=a_{2}$, ou quando $a_{1}$ ou $a_{2}$ é zero, a variável $V$ tem distribuição t-Student multiplicada por uma constante, para outros valores de $a_{1}$ e $a_{2}$ não é fácil obter a distribuição de $a_{1} \chi_{1}^{2}+a_{2} \chi_{2}^{2}$ (Welch, 1936).

Baseado em Student(1908), Welch considera que a curva de tipo III de Pearson é uma aproximação satisfatória para a distribuição de $S=a_{1} \chi_{1}^{2}+a_{2} \chi_{2}^{2}$, sendo a função de densidade dada por

$$
p(S) d S=\frac{1}{\Gamma\left(\frac{1}{2} f\right)} e^{-\frac{1}{2}\left(\frac{S}{g}\right)}\left(\frac{S}{2 g}\right)^{\frac{1}{2} f-1} d\left(\frac{S}{2 g}\right) .
$$


Da equação (26) temos que $S / g$ tem distribuição qui-quadrado com $f$ graus de liberdade, $\operatorname{logo} \frac{Y}{\sqrt{S / f g}}=\sqrt{f g} V$ segue uma distribuição t-Student com $f$ graus de liberdade.

Considerando a distribuição definida em (26) temos que

$$
E(S)=f g \quad \text { e } \quad V(S)=2 g^{2} f
$$

Por outro lado, temos que

$$
E(S)=E\left(a_{1} \chi_{1}^{2}+a_{2} \chi_{1}^{2}\right)=a_{1} f_{1}+a_{2} f_{2} \text { e } V(S)=V\left(a_{1} \chi_{1}^{2}+a_{2} \chi_{2}^{2}\right)=2\left(a_{1}^{2} f_{1}+a_{2}^{2} f_{2}\right)
$$

Igualando (27) e (28), temos que

$$
f=\frac{\left(a_{1} f_{1}+a_{2} f_{2}\right)^{2}}{a_{1}^{2} f_{1}+a_{2}^{2} f_{2}}, \quad g=\frac{a_{1}^{2} f_{1}+a_{2}^{2} f_{2}}{a_{1} f_{1}+a_{2} f_{2}} .
$$

Temos que $f g=a_{1} f_{1}+a_{1} f_{1}=1$, logo a variável $V$ tem t-Student com $f$ graus de liberdade dado por

$$
f=\frac{\left(\sum_{i=1}^{2} \lambda_{i} \sigma_{i}^{2}\right)^{2}}{\sum_{i=1}^{2} \frac{1}{f_{i}}\left(\lambda_{i} \sigma_{i}^{2}\right)^{2}} .
$$

Seguindo o mesmo procedimento podemos generalizar para qualquer $m \geq 2$. 


\section{Referências Bibliográficas}

Aspin, A. A. (1948). An Examination and Further Development of a Formula Arising in the Problem of Comparing Two Mean Values. Biometrika, 35: 88-96.

Ballico, M. (2000). Limitations of the Welch-Satterthwaite approximation for measurement uncertainty calculations. Metrologia, 37: 61-64.

Behrens, W. U. (1929). Ein Beitrag zur Fehlerberechnung bei wenigen Beobachtungen. Landw. Jb., 68: 807-837.

Berkson, J. (1950). Are there two regression?. Journal of the American Statistical Association, 45:164-180.

Berkson, J. (1969). Estimation of a linear function for a calibration line: consideration of a recent proposal. Technometrics, 11: 649-660.

BIPM/IEC/IFCC/ISSO/IUPAC/IUPAP/OIML (1993). International Vocabulary of Basic and General Terms in Metrology (VIM). 2. ed. International Organization for Standardization, Geneva.

BIPM/IEC/IFCC/ISSO/IUPAC/IUPAP/OIML (1998). Guia para Expressão de Incerteza 
de Medição (ISOGUM). 2. ed. Versão Brasileira, INMETRO, Rio de Janeiro.

Brown, P.J. (1993). Measurement, regression and calibration. Oxford: Oxford University Press.

Cheng, Chi-Lun, Van Ness J. W. (1999). Statistical regression with measurement error. London; New York : Arnold: Oxford University Press.

Danzer, K., Currie, L.A. (1998). Guidelines for Calibration in Analytical Chemistry. Part 1. Fundamentals and Single Component Calibration. Pure and Applied Chemistry, 70: 993-1040.

Dietrich, C. F. (1991). Uncertainty, calibration, and probability : the statistics of scientific and industrial measurement. 2nd. Bristol; Philadelphia : A. Hilger.

Fairfield-Smith, H. (1936). The Problem of Comparing the Results of Two Experiments With Unequal Errors. Journal of the Council of Scientific and Industrial Research, 9: 211212 .

Fisher, R. A. (1935). The Fiducial Argument in Statistical Inference. Annals of Eugenics, 6: $391-398$.

Fisher, R. A. (1941). The asymptotic approach to Behrens's integral, with further tables for the d test of significance. Annals of Eugenics, 11: 141-172.

Fuller, W. A. (1987). Measurement error models. John Wiley Sons, New York.

INMETRO (1995). Vocabulário Internacional de Termos Fundamentais e Gerais em Metrologia. INMETRO, Duque de Caxias, Rio de Janeiro. 
International Organization for Standardization (1995). Guide to the Expression of Uncertainty in Measurement (ISOGUM) .

Jeffreys, H. (1940). Note on the Behrens-Fisher formula. Annals of Eugenics, 10: 4851.

King B. (1997). Metrology and analytical chemistry: bridging the cultural gap. Metrologia, 34: $41-47$.

Kyriasis, G. Aibe, V. Y., Alves, J. A. P., Teixeira, R. N., Santo Filho, D. M. E. (2004). Uso do termo probabilidade de abrangência. Nota Técnica do Comitê de Avaliação da Incerteza de Medição - INMETRO.

Lima, C.R.P. (1996). Calibração absoluta com erro nas variáveis. São Paulo: IME-USP. Tese de Doutorado.

Lwin, T. (1981). Discussion of Hunter and Lamboy's 1981 paper. Technometrics, 23: $339-341$.

Polak, E.(1997). Optimization Algorithms and Consistent Approximations. New York: Springer-Verlag.

Shukla, G.K. (1972). On the Problem of Calibration. Technometrics, 14: 547-53.

Sociedade Brasileira de Metrologia (SBM) (2002). Determinando a Incerteza em Medição Analítica. 2. ed. Brasileira - Tradução do Guia EURACHEM /CITAC.

"Student"(1908). The probable error of a mean. Biometrika, 6: 1-25. 
Welch, B. L. (1936). The Specification of Rules for Rejecting Too Variable a Product, with Particular Reference to an Electric Lamp Problem. Supplement to the Journal of the Royal Statistical Society, 3: 29-48.

Welch, B. L. (1947 (a)). The generalization of student s problem when different population variances are involved . Biometrica, 34: 28-35.

Welch, B. L. (1947 (b)). On the Studentization of several variances. The Annals of Mathematical Statistics, 18: 118-122.

Williams, E.J. (1969). A note on regression methods in calibration. Technometrics, 11:189192. 